\title{
Technical Targets - A Tool to Support Strategic Planning in the Subsurface Contaminants Focus Area
}

by

B. B. Looney

Westinghouse Savannah River Company

Savannah River Site

Aiken, South Carolina 29808

This paper was prepared in connection with work done under the above contract number with the U.S. Department of Energy. By acceptance of this paper, the publisher and/or recipient acknowledges the U. S. Government's right to retain a nonexclusive, royalty-free license in and to any copyright covering this paper, along with the right to reproduce and to authorize others to reproduce all or part of the copyrighted paper. 
This document was prepared in conjunction with work accomplished under Contract No. DE-AC09-96SR18500 with the U. S. Department of Energy.

\section{DISCLAIMER}

This report was prepared as an account of work sponsored by an agency of the United States Government. Neither the United States Government nor any agency thereof, nor any of their employees, makes any warranty, express or implied, or assumes any legal liability or responsibility for the accuracy, completeness, or usefulness of any information, apparatus, product or process disclosed, or represents that its use would not infringe privately owned rights. Reference herein to any specific commercial product, process or service by trade name, trademark, manufacturer, or otherwise does not necessarily constitute or imply its endorsement, recommendation, or favoring by the United States Government or any agency thereof. The views and opinions of authors expressed herein do not necessarily state or reflect those of the United States Government or any agency thereof.

This report has been reproduced directly from the best available copy.

Available for sale to the public, in paper, from: U.S. Department of Commerce, National Technical Information Service, 5285 Port Royal Road, Springfield, VA 22161, phone: (800) 553-6847, fax: (703) 605-6900

email: orders@ntis.fedworld.gov

online ordering: http://www.ntis.gov/help/index.asp

Available electronically at http://www.osti.gov/bridge

Available for a processing fee to U.S. Department of Energy and its contractors, in paper, from: U.S. Department of Energy, Office of Scientific and Technical Information, P.O. Box 62, Oak Ridge, TN 37831-0062,

phone: (865)576-8401,

fax: (865)576-5728

email: $\underline{\text { reports@ adonis.osti.gov }}$ 


\section{Technical Targets - A Tool to Support Strategic Planning in the Subsurface Contaminants Focus Area}

Prepared for the Subsurface Contaminants Focus Area (SCFA) by a technical targets team assembled by the Lead Laboratory

Edited by:

Brian Looney

Savannah River Technology Center

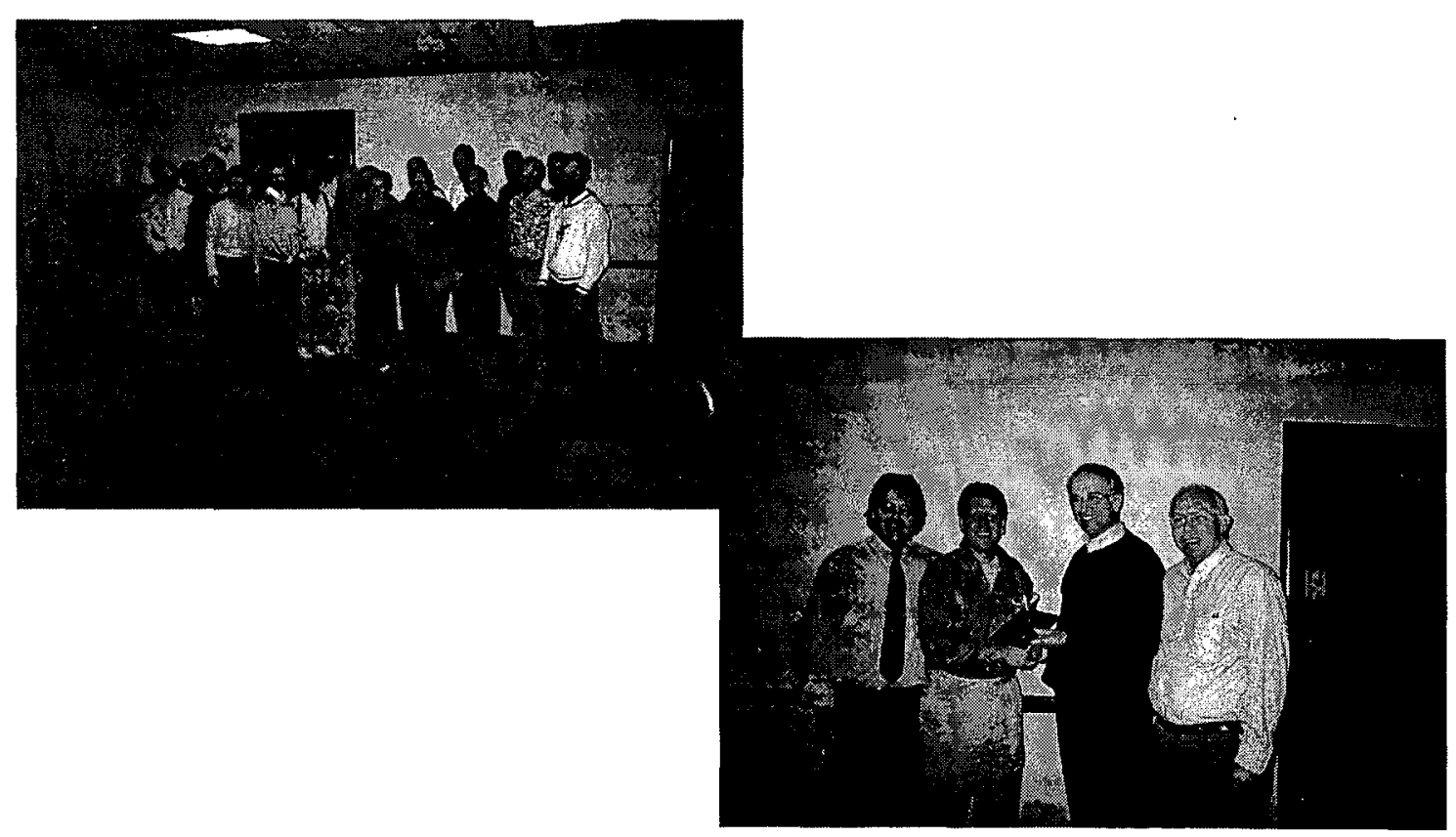

Westinghouse Savannah River Company

Savannah River Site

Aiken, South Carolina 29808

Prepared for the United States Department of Energy under Contract No. DE-AC09-96-SR18500 
The editor gratefully acknowledges the participation and support of technical representatives from across the nation in the technical target process and in the writing and preparation of this document. The team for the initial target development meeting in Golden CO consisted of the following individuals:

\section{TECHNICAL TARGETS PARTICIPATION}

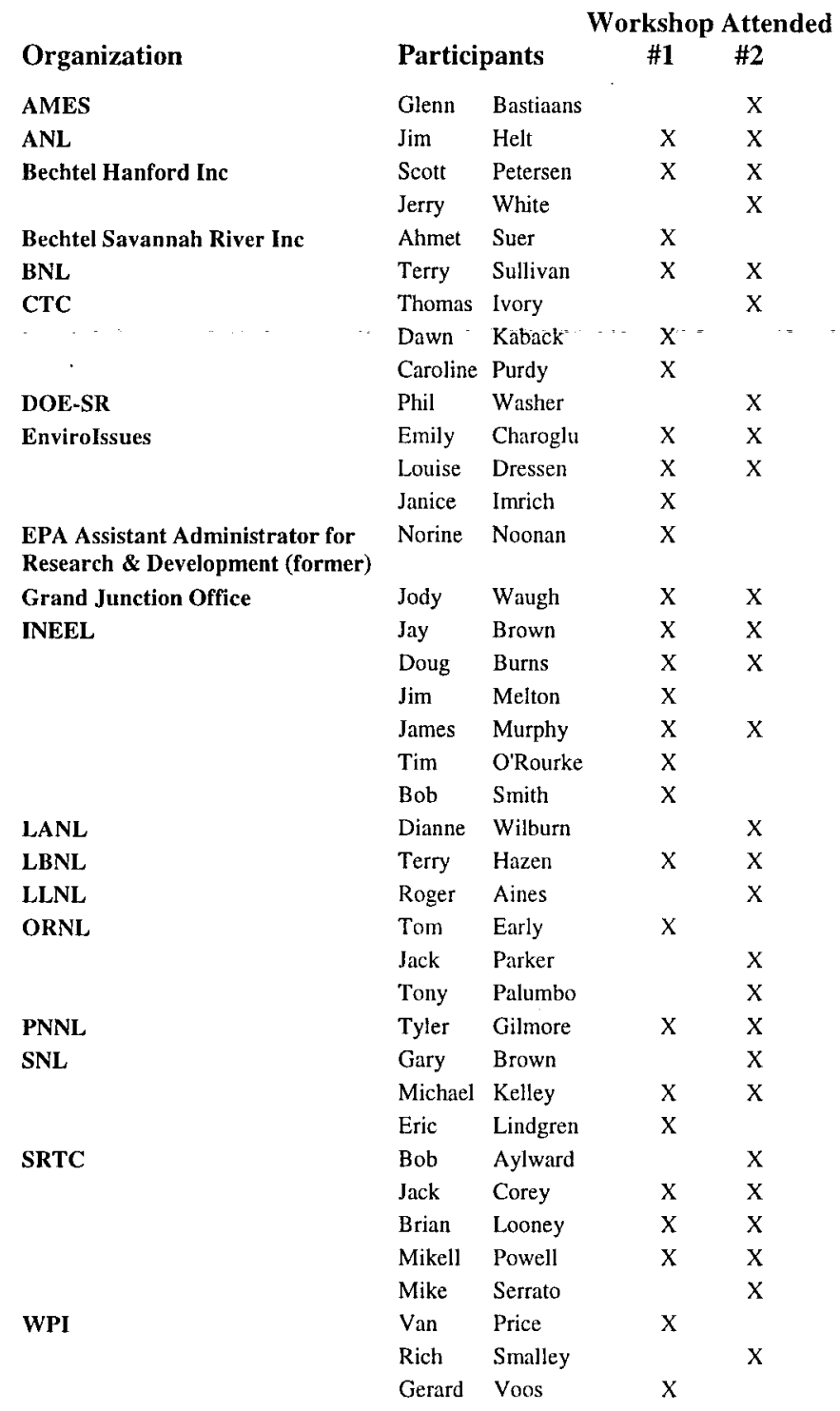

Preparation of this report was coordinated by Westinghouse Savannah River Company (WSRC) for the United States Department of Energy (DOE) under Contract No. DE-AC09-96SR18500. Every effort was made by the editors to assure the accuracy of the contents and interpretation. However, neither the DOE, nor WSRC, nor any of their employees makes any warranty, express or implied, or assumes any legal liability or responsibility for the accuracy, completeness, or usefulness of any information, apparatus, or product, or process disclosed herein, or represents that its use will not infringe privately owned rights. Reference herein to any specific commercial product, process, or service by trademark, name, manufacturer, or otherwise does not necessarily constitute or imply endorsement, recommendation, or favoring of same by the United States Government or any agency; thereof. The views and opinions of the authors expressed herein do not necessarily state or reflect those of the United States Government or any agency thereof. 


\section{Technical Targets - A Tool to Support Strategic Planning in the Subsurface Contaminants Focus Area}

Prepared for the Subsurface Contaminants Focus Area (SCFA) by a technical targets team assembled by the Lead Laboratory

Edited by:

Brian Looney

Savannah River Technology Center

Westinghouse Savannah River Company

Savannah River Site

Aiken, South Carolina 29808

Prepared for the United States Department of Energy under Contract No. DE-AC09-96-SR18500 
\{This page is intentionally "blank", containing only this message and the header\} 


\section{Technical Targets - A Tool to Support Strategic Planning in the Subsurface Contaminants Focus Area}

Table of Contents

Executive Summary

Table of Contents

Overviēw

What is a Technical Target?

1

A Quick Summary of the SCFA Technical Targets Developed in Golden 2

Introduction

Overview of Technical Target Effort

General Principles in Developing Prospective Targets

Content of a Technical Target

Results and Recommendations

Appendix A One Page "Study Guide Style" Summaries of SCFA Technical Targets Developed in Golden CO

Appendix B Full Texts of the SCFA Technical Targets Developed in Golden CO

Appendix C Information on the Inaugural Meeting in Golden CO 
WSRC-RP-2002-00077

Revision 0

Page iv of $x$

\{This page is intentionally "blank", containing only this message and the header\} 


\section{Technical Targets - A Tool to Support Strategic Planning in the Subsurface Contaminants Focus Area}

\section{List of Figures}

1. Technical Targets in the Strategic Management Hierarchy 8

2. Anatomy of a contaminated site 11

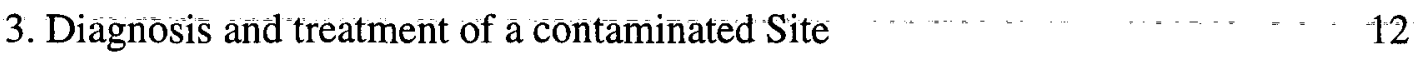

4. Cutaway diagram showing the 3D Structure of a real groundwater plume 13

5. Environmental restoration is performed by blending technologies from the five topic areas previously identified by SCFA

6. Draft technical targets and relationships 16

7. Annotated outline of the technical targets 
WSRC-RP-2002-00077

Revision 0

Page vi of $x$

\{This page is intentionally "blank", containing only this message and the header 


\title{
Technical Targets - A Tool to Support Strategic Planning in the Subsurface Contaminants Focus Area
}

\author{
Abbreviations and Acronyms
}

CMST - Characterization, Sensor, and Monitoring Integrated Program

DOD - Department of Defense

DOE - Department of Energy

DNAPL - dense nonaquesous phase liquid

DUS - Dynamic Underground Stripping

EM - Environmental Management

EMAB - Environmental Management Advisory Board

EMSP - Environmental Management Science Program

EPA - Environmental Protection Agency

ESTCP - Environmental Security Technology Certification Program

$\mathrm{HQ}$ - Headquarters

IP - Industry Programs

ITRD - Innovative Treatment Remediation Demonstration

RTDF - Remediation Technologies Development Forum

SCFA - Subsurface Contaminants Focus Area

SERDP - Strategic Environmental Research and Development Program

STCG - Site Technology Coordination Group

TechCon - \{DOE $\}$ Technology Connection

UP - University Programs

USGS - United States Geological Survey 
WSRC-RP-2002-00077

Revision 0

Page viii of $x$

\{This page is intentionally "blank", containing only this message and the header\} 


\section{EXECUTIVE SUMMARY}

The Subsurface Contaminants Focus Area (SCFA) is supported by a lead laboratory consisting of technical representatives from DOE laboratories across the country. This broadly representative scientific group has developed and implemented a process to define Technical Targets to assist the SCFA in strategic planning and in managing their environmental research and development portfolio. At an initial meeting in Golden Colorado, an initial set of Technical Targets was identified using a rapid consensus based technical triage process. Thirteen Technical Targets were identified and described. Vital scientific and technical objectives were generated for each target. The targets generally fall into one of the following five strategic investment categories:

- Enhancing Environmental Stewardship

- Eliminating Contaminant Sources

- Isolating Contaminants

- Controlling Contaminant Plumes

- Enabling DOE's CleanUp Efforts

The resulting targets and the detail they comprise on what is, and what is not, needed to meet Environmental Management needs provide a comprehensive technically-based framework to assist in prioritizing future work and in managing the SCFA program. 
Revision 0

Page $x$ of $x$

\{This page is intentionally "blank", containing only this message and the header 
Overview

A new strategic planning tool - Technical Targets - has been developed to support management and decisionmaking in the Subsurface Contaminant Focus Area (SCFA). Technical Targets are produced by a cost-effective, intense and focused technical consensus process. The process, modeled after highly successful SCFA technical assistance efforts and the broadly representative SCFA Lead Lab, emphasizes creativity and responsiveness to critical science and technology needs. A pilot Technical Targets effort was held in Golden, Colorado from July 23-July 27, 2001. The workshop was successful - both in validating the process and in generating an initial portfolio of SCFA Technical Targets for use in strategic planning and communication. The participants in Golden included technical representatives from across the DOE complex and from other agencies and organizations. They were asked to identify and highlight the critical technology and basic science needs that are embodied in the approximately 339 raw end-user needs statement and in other resource and planning documents (e.g., the Vadose Zone Roadmap). The participants worked collaboratively and generated 13 Technical Targets and short descriptions. Each of the 13 targets has a unique character and addresses a particular set of science, technology, and policy issues. We summarize the targets below and have attached the complete targets and descriptions developed by the team.

Central themes that appeared in many of the targets included:

- selecting actions that work in concert with natural conditions rather than working against nature or trying to hold off nature

- matching the cost and aggressiveness of remedial actions to the degree of risk and potential benefit (and matching the selected technology implementation to the environmental setting and contaminant distribution)

- using a holistic approach to solving end-user needs - improving technical and cost performance by integrating actions that are artificially separated in current paradigms

Based on the activities during the week, the participants recommended editing, revising and finalizing the Technical Targets developed at the workshop and transmittal to our sponsor, SCFA, for use in ongoing strategic planning efforts. A second workshop was then held, also in Golden, where we finalized the draft Technical Targets.

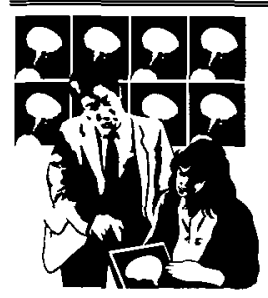

The best way to describe the purpose of Technical Targets is by analogy to medicine. The 339 end-user needs are analogous to symptoms described by a patient. In developing the Technical Targets, a team of technical specialists examines the 339 symptoms and attempts to organize them and describe the 10 to 20 underlying diseases that are causing most of the problems. 


\section{A Quick Summary of the Initial Set of SCFA Technical Targets Developed in Golden}

To help organize and explain the targets, we defined five strategic investment categories:

- Ensuring Environmental Stewardship

- Eliminating Contaminant Sources

- Isolating Contaminants

- Controlling Contaminant Plumes

- Enabling DOE's Clean-Up Efforts

Each of the proposed Technical Targets was assigned to a strategic investment category. The categories and targets are summarized below. In the full targets, technical detail and bounds are provided as part of the vital technical objectives to assist in decisionmaking and defining/measuring progress toward the target. Other information in the full target incliudes a summary of the needs addressed and the relevance of the proposed activities and an assessment of science and technology development status.

\section{Ensuring Environmental Stewardship}

This strategic investment area addresses critical needs to develop improved paradigms for efficiently protecting and restoring the environment. Importantly, these targets attempt to examine and develop wholly new approaches that minimize risks and costs associated with past contamination, while concurrently minimizing the adverse impacts associated with the selected restoration activities. In the initial effort, the target development team identified two technical targets that would significantly address this strategic investment area in an integrated, efficient and constructive manner.

Improving the Technical Basis for Setting Remediation Goals

This target recommends working with end users, regulators and stakeholders to develop a more holistic approach for setting goals. This target identifies several specific technical advances that would improve decisionmaking and recommends rapidly integrating the advances into decision support resources for beneficial use in baseline activities. Vital development themes with particular promise to DOE include: 1) prioritizing the desirability of end states, 2) time phased decisionmaking, 3) technically defensible assessment of collateral damage, and 4) accounting for baseline risk for natural elements and synergistic effect in contaminant mixtures.

Methods to Verify and Validate Performance

This target recognizes the need to develop distinctly different technologies for short term performance monitoring versus long term monitoring. The former relies primarily on improved sensor development, emplacement and data integration, while the later relies primarily on developing wholly new verification paradigms to allow monitoring for decades or centuries. Large scale methods (remote sensing, geophysics) and integrating methods (flux or release measurements), among others, appear promising for long term monitoring. The team recommended a technology development focus on long term monitoring and a technical assistance focus on performance monitoring. 


\section{Ėliminating Contaminant Sources}

The targets in this strategic investment category relate to the technologies that actively and assertively eliminate risks associated with contaminant source areas. The two targets recognize that there are substantial differences in technology objectives for metals and radionuclides (which can be stabilized or removed) versus for organic contaminants (which preferably can be destroyed). These targets are critically important because source areas must be eliminated or stabilized for any subsequent plume control and/or long term risk reduction techniques to be successful.

Organic Source Zone Stabilization and Treatment

This target advocates continuing the past SCFA progress in moving through the National Academy of Sciences' chart of remediation challenges from less difficult to more difficult. Thus, the target recommends a central strategy of collaboration with other agencies to advance practical application of organic source zone treatment methods. The specific focus for future activities is on enhanced removal and in-situ destruction technologies for conditions that are more difficult than those already addressed by SCFA (fractured systems and low permeability media). Another vital objective highlighted in this target is development and selection of techniques that are compatible with a transition to less aggressive and more passive polishing of the site - for example, technologies that do not foreclose on biological remediation because they cause long term sterilization of the subsurface. A final concept described in the target is the need for characterization tools to designate and delineate the source zone so that expensive and aggressive cleanup methods are not applied in areas where they are not needed.

\section{Metals and Radionuclide Source Zone Stabilization and Treatment}

The focus of this target is on treatment/removal approaches and techniques that overcome the issues of complex waste near the source and facilitate transition to more traditional (i.e., lower cost) techniques and/or to document that residual is stabilized to protect human health and the environment. This target is quite different than the one related to organic source zones because of the fundamentally different nature of radionuclides and metals. In this case, technology development leading to stabilization is emphasized for metals where transport is slow and exceedances of standards (e.g., groundwater concentrations) are relatively low. Important, but exceptional, cases where geochemistry is highly perturbed (high level waste tanks at Hanford, uranium in groundwater at Oak Ridge) are important and the issue of understanding and manipulating chemical speciation is a vital theme. The target recognizes that physical containment is a viable option for relatively small source zones. 


\section{Isolating Contaminants}

The targets in this strategic investment category address uncertainties and improved technologies for physically isolating contamination-either as an interim or permanent solution to a contaminant source. The targets in this category recognize that isolation and barrier technologies have been, and will continue to be, a primary tool in addressing environmental contamination. As a result, progress on these targets will positively impact both near term and future environmental management effectiveness in a realistic real-world scenario.

Advanced Sustainable Containment Systems

Devise/Develop containment systems based on natural analogs. Develop containment systems that have robustness based on fundamental theoretical processes ("long life") and which integrate with related remediation activities. Properly applied and monitored, physical containment and barriers will remain a central activity in DOE environmental management for the foreseeable future. Advancing the science and technology base relatively rapidly is particularly important to closure sites that need to implement and document such systems in the next several years.

Integrated Storage-Treatment Concepts - "Smart Containment"

Develop integrated systems so that solid hazardous and/or radioactive waste is stabilized or detoxified during a period of containment or isolation. To support such a strategic development, potential treatment processes (delivery systems and their compatibility with the isolation systems) must be evaluated, as well as methods to monitor treatment progress and document when the waste containment is no longer needed. This target is a specific example of a technical approach that crosses traditional administrative boundaries and which implements the NAS/NRC recommendation of building multiple defenses into long term stewardship systems. The technical target team felt that this type of target had a unique character that may encourage a crosscutting strategic investment and may be a desirable style of target to consider for future development.

\section{Controlling Contaminant Plumes}

These targets address the general need to eliminate or mitigate risk associated with contaminants that have already spread into the environment. The concentration of these dispersed contaminants is much lower than in the source area so that entirely different technological approaches are needed. While all of the targets identified in this particular strategic investment area emphasize a holistic solution to dispersed plumes, each target focuses on a different aspect of the problem. The targets address general needs associated with subsurface/surface contaminants, as well as a particular challenging $D O E$ related need associated with a specific contaminant (tritium). 
Effective and Sustainable Technological Solutions for Contaminant Plumes

Several vital scientific and technical objectives remain: 1) optimizing active treatment systems, 2) understanding the relationship between performance and the completeness of source zone treatment, 3 ) developing technologies that transition more quickly to monitored natural attenuation (through emplacement of long term reagent materials, through identification of measurable natural processes that destroy, stabilize, or detoxify dilute plume contaminants), and 4) developing design approaches and viable monitoring strategies. This topic is viewed as critical to future DOE environmental stewardship performance - with technology development emphasis on objectives 2 through 4 .

\section{Tritium Management and Risk Reduction}

Tritium is a contaminant with unique challenges. The vital objectives for this target include: 1) "cost effective" tritium treatment technologies, 2) alternate tritium remediation strategies to reduce releases, exposure and risk, and 3) tritium characterization and monitoring tools, with particular emphasis on identifying plume arrival or tritium fluxes and risk. The target sets an approximate standard for what is meant by "cost effective" to assist in evaluating if a proposed technology represents an advance over the baseline.

\section{Enabling DOE's Clean-Up Efforts}

This strategic investment area comprises two distinct and critically important types of activities: engineering technologies and basic science developments. The targets were selected based on the number and types of needs described by end users and by the various needs documents developed by the National Academy of Science and other expert panels. Progress in the identified target areas is critical to meeting short term and long term environmental restoration goals.

\section{Subsurface Access and Delivery}

The three most important objectives highlighted in this cross cutting target are: 1) access under obstructions, 2) delivery of fluid for treatment or containment, and 3) deep access.

\section{Techniques and Technologies that Support Characterization}

Vital themes identified and described for this target include: 1) improvement of noninvasive characterization technologies, 2) measurements at various scales (point and volume integrated) to support multiple objectives, 3) development of field deployable systems, and 4) integration of multiple types of characterization data.

\section{Biogeochemical Processes that Determine Contaminant Fate}

The effective implementation of remediation strategies and natural attenuation for the cleanup of DOE sites depends on understanding critical chemical, physical and biological processes. Particular important research themes include: 1) redox conditions that affect biogeochemical processes, 2) anthropogenic influences on the biogeochemistry of natural systems and extreme environments, and 3) coupling and scaling issues. 
Strongly Heterogeneous Systems

This target emphasizes the significance of the issue and challenge and the significant historical investment and research. Based on the state of science and art, there is a vital need to develop alternate, non-deterministic approaches (similar to weather modeling) as a critical path in the future.

\section{Advanced Environmental Modeling}

The vital needs associated with modeling fundamental environmental processes are: 1) identifying and filling fundamental environmental process knowledge gaps (as described in the vadose zone road map, for example), 2) improving uncertainty quantification techniques, and 3) developing practical-comprehensive tools and approaches for performing modeling. The team felt that near term transitioning modeling techniques from state-of-art to state-of-practice is important to support closure sites and for technical assistance. 


\section{Introduction}

SCFA has initiated a "Technical Targets" development process to support its strategic planning process. The process is a venue for the technical community to provide insight and strategic thinking to support SCFA management. The process is designed to generate Technical Targets. These capture and prioritize needs based on technical considerations and experiences elicited from a highly qualified, broadly representative team. To test the concept, SCFA sponsored the first Technical Targets development meeting in Golden, Colorado (July 23-27, 2001) and a followup meeting (November 27-29, 2001) to finalize the product. The objectives of these meetings were: 1) to solicit input on the Technical Targets concept and proposed process, 2) to generate an initial draft set of technical targets for use by the Focus Area in near term strategic planning efforts, 3 ) to assess the technical target process, and 4) to develop recommendations on prioritizing and using the targets in strategic planning. These activities were completed and are documented in the following sections. The most challenging aspect of the work was developing the logic and then defining the actual targets to be developed. This process generated a small number of targets, each of which centers on a set of unique and important themes. The logic and the resulting target definitions were interesting and particularly important and are addressed in significant detail.

\section{Overview of Technical Target Effort}

Over the past several years, SCFA has successfully developed and deployed technology to enhance DOE's environmental management and stewardship activities. The successes of these efforts have been measured by simple counting (deployments and approximate cost savings) and by showing that research efforts correspond with one or more of the hundreds of raw needs statements generated by one of the site technology coordination groups (STCGs) in the DOE complex (the "end-user needs"). Investment prioritization has been based in large part on counting the raw number of end-user needs in the various work packages. SCFA held a brainstorming meeting to determine the best approach to build on past success and to improve future success. In this "2001 Beyond Breakthrough Meeting", SCFA devised the concept of "Technical Targets" as an important tool to help prioritize and select work and to improve investment efficacy and returns in the future.

In 2001, the number of end-user needs statements sorted into the purview of SCFA increased to 339 . The Technical Targets proposal recognized that managing an applied research and development program to 339 raw end-user needs, written at varying levels of detail and with varying local and near-term drivers, is difficult. To provide perspective and balance, SCFA proposed utilizing the expertise in the technical community to help organize and identify fundamental technical issues/themes embodied in the end-user needs. If the 339 end-user needs are the "symptoms" in a medical analogy, then the targets are the 10 to 20 "diseases".

The result is a portfolio of "diseases" and short clear descriptions of the most important causes and characteristics. The portfolio is a tool to help SCFA:

- solicit and select the most useful future science and technology activities, 
- communicate the program vision and strategies to end users, executive organizations (DOE-HQ, Congress and the like), oversight and advisory groups (National Academy of Sciences and the like), other federal agencies, and nongovernmental organizations and individuals,

- develop improved metrics for program performance and value. Future metrics could be designed to assess progress in curing the diseases rather than a simple counting of projects that address symptoms.

Figure 1 shows the technical target concept as it fits within an overall hierarchy that links the real-world needs with the various levels of detail needed to effectively manage an environmental R\&D program. Technical Targets represent a new tool to help manage the program. They are written at an intermediate level of detail so that they have clear specificity in what needs to be done. Higher tier generally address the question of why the technical work is needed - important in justifying the program but too broad to be the basis of an effective and focused research portfolio. The detailed needs vary in focus and specificity, but they generally describe specific cases of how a technology would help useful to fix specific problems but difficult to use as a management tool because of the large amount of specific information.

\section{Technical Targets in the Strategic R\&D Management Hierarchy}

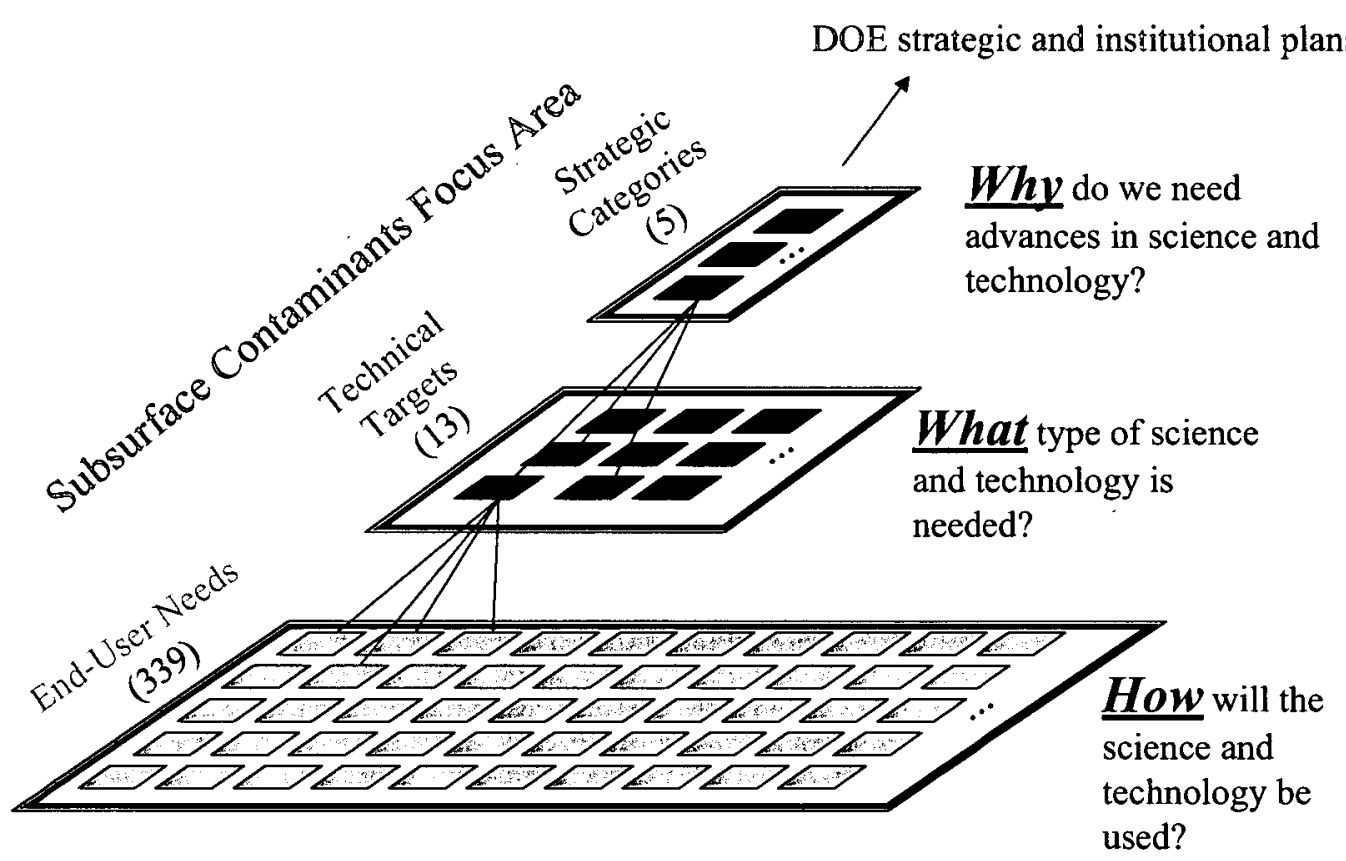

Concept adapted from the hierarchy of models by Boris Fabyshenko

Figure 1 
We emphasized technical creativity and vision when developing and optimizing the targets in an attempt to broadly address end-user needs within a resource constrained portfolio. Technical Targets are not intended to be a comprehensive strategic planning activity - they recognize that resources are limited and attempt to highlight vital activities instead of an unconstrained complete list of needs and uncertainties. The Technical Targets Process was designed to be relatively inexpensive and responsive. It was coordinated by the SCFA Lead Lab and was modeled after the successful Lead Lab technical assistance programs within SCFA. The process is not a "roadmapping" process and does not generate a detailed and formal management plan. The resulting Technical Target portfolio, however, is an important input to formal planning and a tool to assist SCFA management with developing strategies and communicating their vision.

The initial Technical Target development workshop in Golden, Colorado was an intense focused effort by a group of 22 technical participants. In general, the meeting addressed the following items, in turn: defined and described the overall Technical Target Concept, developed a list of prospective targets, agreed on general structure of a target, authored the prospective targets, and evaluated the workshop and draft targets to assess potential value to SCFA. A unique, and highly successful, feature of the workshop was the approach developed to generate the targets. Each target was initially discussed by the entire group. In this initial discussion, the participants were encouraged to rapidly describe their most creative ideas for technical issues/themes related to the topic. The group's initial ideas were then used by a small team of approximately three participants to generate a detailed outline for the proposed target content. The outline was the basis for a second discussion by the entire group to determine if there was general consensus on the approach being described. The small team then wrote the entire target for inclusion in the draft package. In this way, approximately 7 targets could be written at one time. The two general discussion sessions ensured that the creative thoughts from the entire group were available for improving the targets and provided a check and balance on potential biases. The small groups were efficient in the writing process. All of the fourteen proposed targets were addressed (in two batches) using this approach. A one page summary and the competed texts of the technical targets are provided in Appendix A and Appendix B, respectively. A detailed description of the first meeting is provided in Appendix C. The Technical Targets approach and draft targets were validated at a broad-based technical program reformulation meeting. DOE site managers, DOE laboratory managers, DOE headquarters staff, and end users all participated in this meeting. The target concept was supported and the Lead Laboratory was encouraged to move forward in the process. A second technical workshop was then held, also in Golden, where the draft Technical Targets were finalized.

\section{General Principles in Developing Prospective Targets:}

Many different approaches to defining the titles and topics for Technical Targets are possible, and several of these would be viable. The most important element in the process was developing targets where end-user needs cluster into vital development themes/topics. These themes/topics been called heavy hitters, technical choke-points, and similar designations in past planning efforts. The remaining criteria in developing 
the target list were intended to encourage technical insights and elicit a defensibletechnically-based subset of a comprehensive-unconstrained program to help guide future decisionmaking. The following list of principles and resources were used in developing the Technical Targets.

\section{Principles used to develop Technical Targets}

- Develop targets in which technical issues can be easily highlighted because they are central (or unifying) to the target. This will assist in clear and concise writing.

- Develop targets that capture and address end-user needs in a demonstrable manner.

- Build on straightforward conceptual models of effective ways to systematically perform environmental activities.

- Develop targets that will support strategic management of the Focus Area.

- Develop targets that recognize that this particular strategic planning process needs to be rapid, efficient and flexible.

- Develop targets that will encourage investment in a diverse technically-based portfolio.

- Develop targets that contain sufficient detail to allow development of metrics and to support writing of future requests for proposals and in selecting work to meet needs.

- Develop targets that recognize the unique role of SCFA to bridge Basic Science, Applied Science and Deployment.

- Develop targets that encourage healthy competition (comparing and contrasting) alternative solutions rather than organizing along disciplinary lines. Since SCFA is the organization where the basic to applied science transition occurs, alternative solutions to problems from different disciplines need to be compared rather than stovepiping and allocating funding to each discipline for all problems.

- Consider individual sites, past strategic planning, work packages, and related integrated needs efforts as resources.

\section{Partial list of resources used to develop targets}

- STCG Needs Statements

- Previous needs summary and roll up efforts (e.g., GJPO 104)

- National and individual site roadmapping activities (e.g., vadose zone roadmap)

- SCFA Work Packages

- EM R\&D Strategic Plan (Draft 6/15/01)

- SCFA Product Line Planning activities

- Vadose Zone Science and Technology Solutions

" Policy Documents (e.g., "Wasteland to Wilderness", and the like)

In the initial effort, the team settled on Technical Targets based a combination of enduser objectives, contaminant type, and contaminant level using the conceptual models described below. This list of targets led to relatively straightforward descriptions and unique-identifiable-vital scientific and technical objectives within each target. 
Figure 2 depicts a conceptual diagram of a contaminated site that has impacted its surroundings - in this case, the underlying soil and groundwater. The three ovals - the source zone, the primary contaminant plume, and the dilute fringe - represent different portions of the impacted environment that each has a different character. The source zone contains significant contamination in concentrated and hazardous forms. The source zone can contain materials such as undissolved organic liquids (oils, fuels or solvent), strong acids or bases, high levels of radiation, and/or toxic chemicals or elements. The second oval, the primary contaminant plume, is comprised of contaminated groundwater or vapor than carries pollutants at lower levels, but levels that still represent a potentially significant present or future hazard. The third oval, the dilute fringe, contains contamination at relatively low concentrations, but in large volumes of water.

\section{Anatomy of a Contaminated Site}

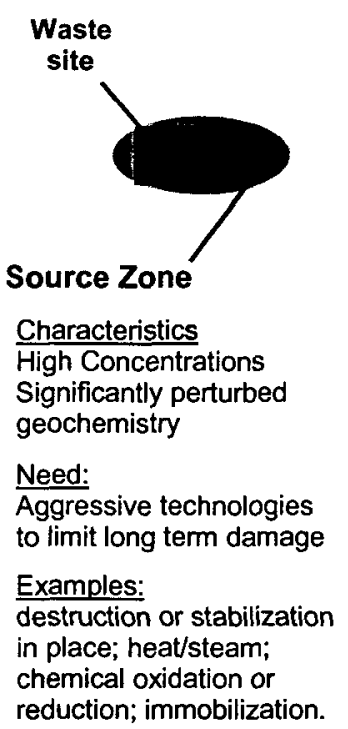

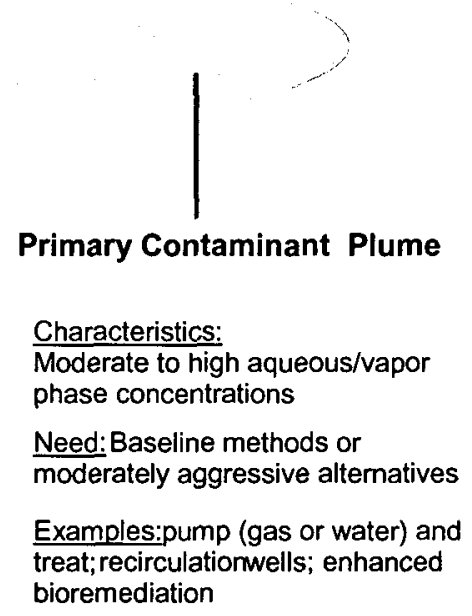

Figure 2

Efficient and effective environmental clean up requires matching the character of the clean-up and stabilization methods to the character of the target zone of contamination. Thus, aggressive and relatively expensive methods are often appropriate for the source zone, baseline methods are often good for the primary contamination zone, and various methods based on natural processes are often best for the dilute fringe. Figure 2 identifies several example technologies that are appropriate for each of the ovals. 
In Figure 3, the cost basis for the typical clean up technologies is identified. In the source zone, stabilization and removal methods are normally priced in terms of volume of soil or amount of contaminant in the treatment zone (\$ per cubic yard, $\$$ per pound and the like). The reference source zone technologies require aggressive access and subsequent use of targeted energy or chemical reagents. It is clear that in the source zone it is important to characterize the site in such a way that the location requiring treatment is delineated as carefully as possible. This characterization approach will reduce costs by focusing energy or reagent to areas where they are needed. Equally important, however, is a desire to minimize any undesired negative impacts (wasting energy, harming microbiological populations, etc.) associated with using aggressive remedies on regions without source level contamination.

\section{Diagnosis and Treatment of a Contaminated Site}

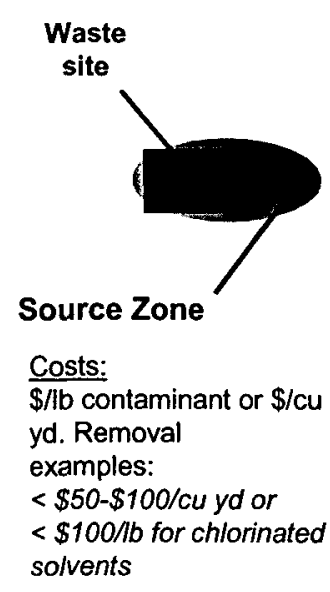

hot spot characterization reduces cleanup volume

\author{
| \\ Primary Contaminant Plume \\ Costs: \\ \$/treatment volume (gallon/cu $\mathrm{ft}$ ) \\ example: \\ $<\$ 0.5-\$ 10 / 1000$ gallons \\ zone of capture characterization \\ needed, optimize extraction to \\ reduce treatment volume
}

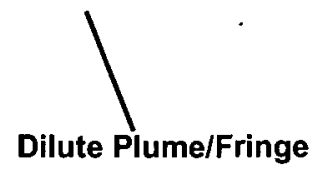

Costs:

Operation and

maintenance costs $\$ /$ time

mass transfer and flux characterization needed

\section{Figure 3}

In the primary contaminant plume, treatment technologies are normally priced in terms of the amount of water (or vapor) treated ( $\$$ per gallon and the like). Thus, the goal of characterization is to define the flow directions and general plume structure to allow the most contaminant to be treated in the fewest "gallons." Figure 4 illustrates an important final extension to our simplified conceptual model. This diagram of the primary contaminant plume at a DOE site (the Metals Fuel and Target Fabrication Facility, M Area, at the Savannah River Site (SRS)) shows that contamination moves in response to many factors - contaminant release location and type, geology, sources and discharges of water, and many others. The resulting contaminated soil and groundwater zone occupies a complicated three-dimensional shape rather than the simple ovals previously described. This complexity must be recognized when developing and implementing technologies for both characterization and clean up of the primary contaminant plume. 


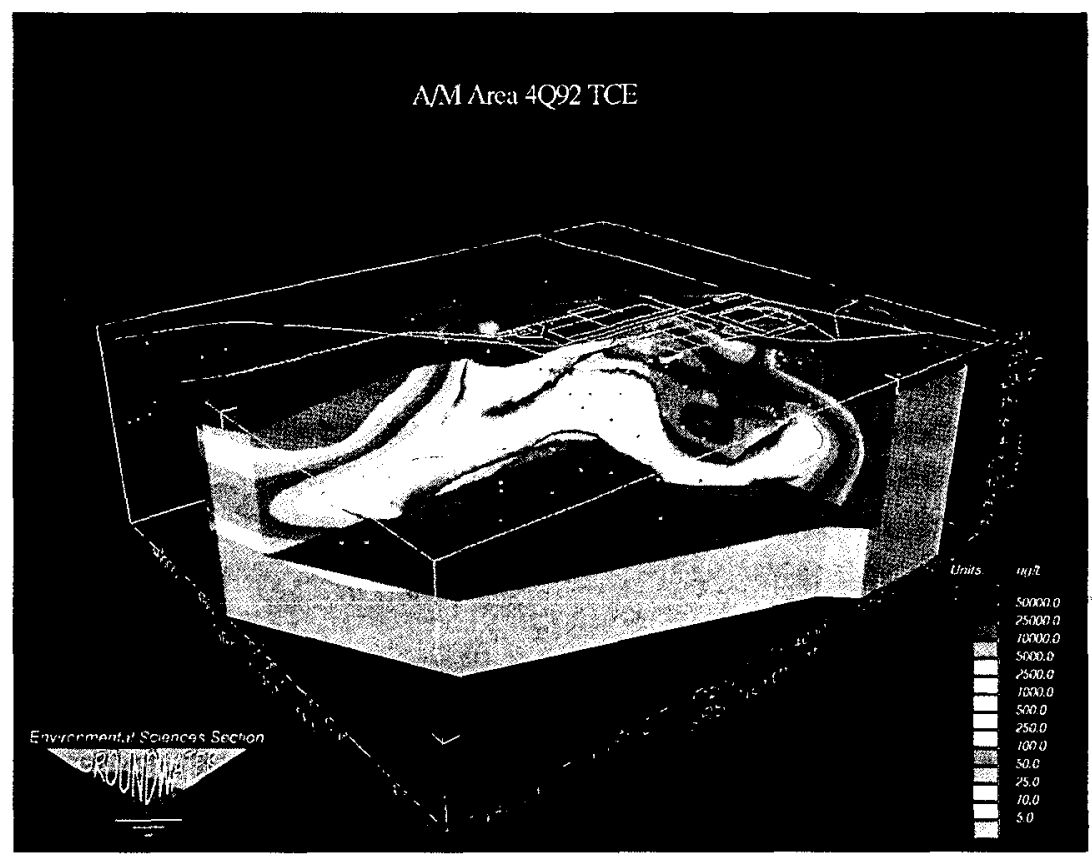

\section{Figure 4. Cut-away diagram showing the 3D structure of a real groundwater plume}

The dilute fringe contains low concentrations of contamination in large volumes of water. Thus, the best technologies for this zone are those that are priced in terms of time ( $\$$ per year and the like). To be successful, these technologies must rely on natural-sustainablemeasurable processes. This class of technology has gained recent regulatory support under the terminology "monitored natural attenuation". For the dilute fringe, technology selection should be directed towards understanding the contaminant destruction and stabilization capabilities of native species and natural populations. A second step is identifying engineering interventions, if needed, to maximize the performance and to ensure that the attenuation process will operate for extended periods. A critical requirement for these technologies is development of logical and cost-effective monitoring strategies.

The three zones depicted in Figure 2 are present at contaminated sites of all sizes. At a "mom-and-pop" gas station, the entire contaminated zone - all three ovals - might occupy a portion of a city block. At a large industrial facility like the M Area at SRS, the contaminated zone can extend over a few square miles. The size of a problem impacts how distinct the actions to address the different zones need to be. Time is also a factor. Concentrations change as cleanup progresses, so that dilute fringe technologies become appropriate for polishing areas that were formerly at higher concentrations.

For the past several years, the SCFA approach revolved around development, demonstration, and deployment within five key categories. These categories, which were called "strategies", were well conceived and were another important conceptual model 
incorporated into the development of prospective Technical Targets. An appropriate combination of all five strategies is required in every environmental management situation across the entire range of possible conditions. Figure 5 shows the five SCFA identified strategies.

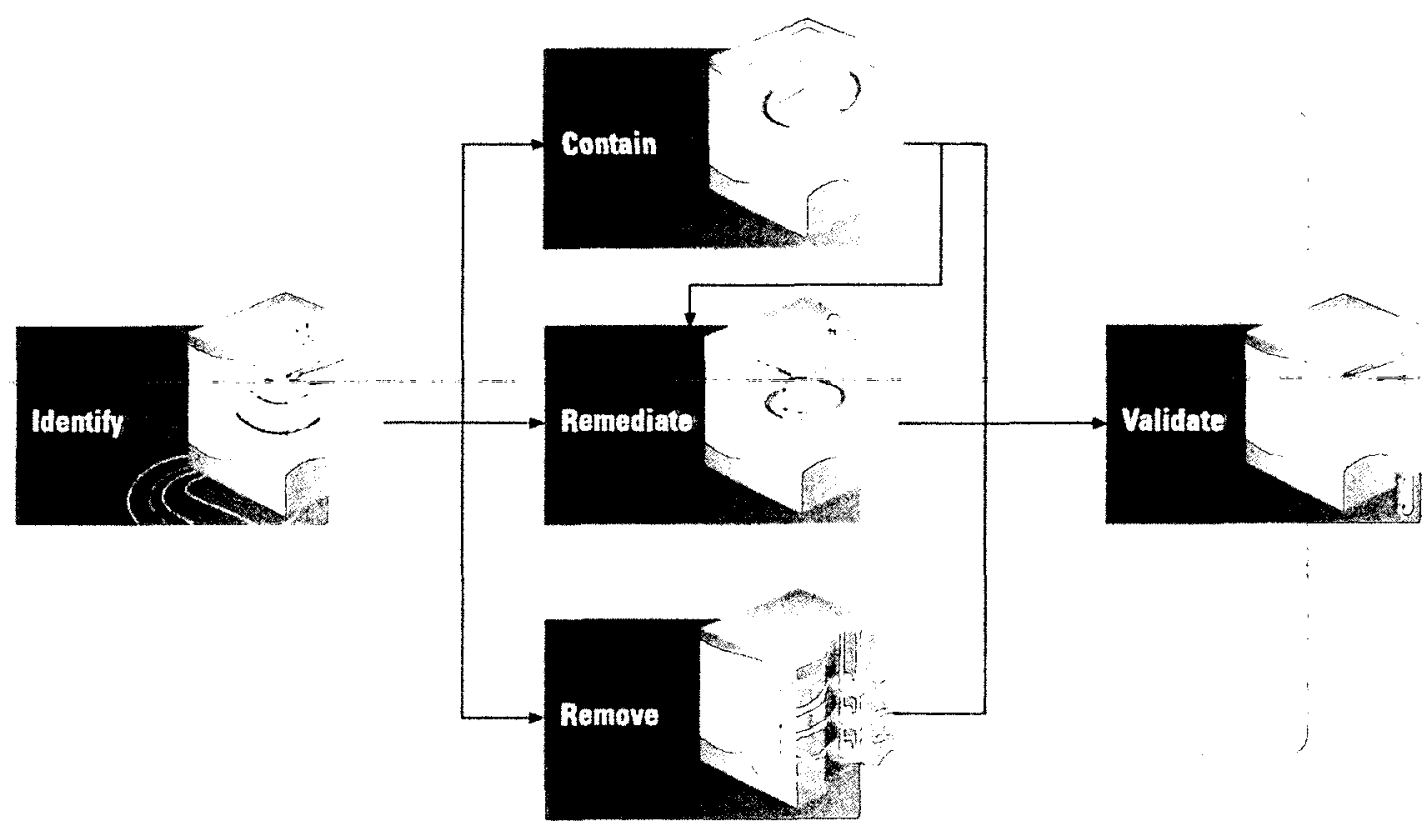

Figure 5. Environmental Restoration is performed by blending technologies from the five topics areas previously identified by SCFA.

A final concept that was emphasized in the development of targets was the need to encourage a holistic evaluation of cleanup actions to avoid missing a technical opportunity. For example, can a barrier be designed that also treats and detoxifies the waste rather than simply isolating it forever?

In the end, the team determined that an appropriate set of technical targets could be built around the relatively unique requirements, actions and challenges in the various plume regions (source zone, primary plume and dilute plume). Further, the team felt that the nature of the technical issues was different for organic source areas versus metal/radionuclide source areas, but that contaminant specific issues become less important in the primary and dilute areas. We identified a few contaminant topics (e.g., tritium) that have unique features, as well as several crosscutting targets that embody important science and technology development themes. To help organize and explain the targets, we defined five strategic investment categories: 
WSRC-RP-2002-00077

Revision 0

Page 15 of 20

- Ensuring Environmental Stewardship

- Eliminating Contaminant Sources

- Isolating Contaminants

- Controlling Contaminant Plumes

- Enabling DOE's Clean-Up Efforts

Each of the proposed Technical Targets was assigned to a strategic investment category. The list of Technical Targets and how they relate to each other in a overall sense are depicted graphically in Figure 6. The targets can generally be classed into groups - those that address specific-required environmental response actions, and various types of crosscutting targets that provide the holistic basis for taking actions or that are needed for all of the various types of response actions. For each target, the team felt that a unique and measurable description of the necessary class of action could be developed.

A particularly interesting and notable event from the meeting was the presentation by Bechtel Corporation of an independent activity that they were performing to summarize their assessment of the most important Environmental Restoration needs. Bechtel is a contractor organization at five major DOE sites, so their ranking and summarization of environmental needs across the country represented an interesting and critical check of our process by an end-user group. The draft Bechtel corporate list of summary environmental need topic areas ("targets") was almost identical to the independently proposed technical target list generated prior to Golden and to the final list developed in Golden. The Bechtel list is as follows:

- Surface Cover Design and Monitoring

- In-Well Groundwater Monitoring

- In-Situ Stabilization of Contaminants

- Tritium Monitoring and Remediation

- VOC Remediation

- Data and Scientific Knowledge to Help Understand Contaminant Transport and Assess the Potential for Deploying Passive Remediation for Monitored Natural Attenuation

- Cost Effective Access to the Subsurface, Especially the Deep Subsurface

- DNAPL Characterization and Remediation.

It is clear from the general concordance between the lists that the Technical Targets as proposed should be generally supportive of end users. In general, differences between the Technical Targets and the Bechtel Corporate list can be attributed to two factors:

1) our objective to develop targets that address needs at all DOE sites (e.g., some of the closure sites as well as Bechtel sites), and

2) differences in emphasis and approach between the two efforts. 


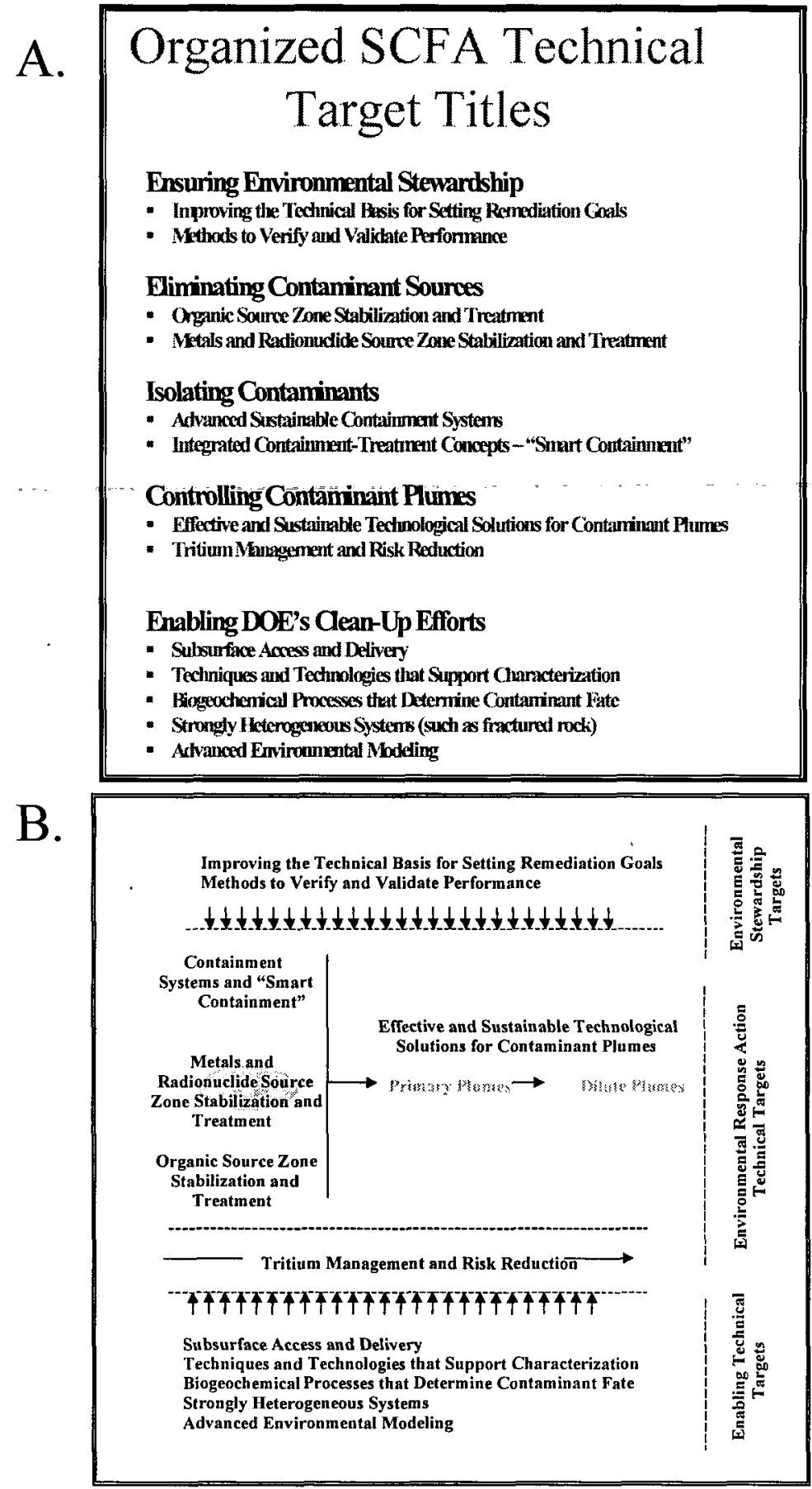

Figure 6. SCFA Draft Technical Targets and Relationships. A) Sorted by strategic investment category, and B) graphical representation relating targets to environmental restoration activities 
Figure 7 is an annotated outline of the content of each technical target.

\section{Technical Target}

\section{Title:}

\section{Summary of Need(s):}

Brief statement that distills and summarizes a group of related needs.

\section{Relevance}

Why is this important. Contains data on number of sites, current costs and potential savings if the target is successfully addressed.

\section{Status:}

This section recognizes previous work both inside DOE and by other agencies, private industry etc. It sets the stage for the bounds in the next section. Vital Scientific and Technical Objectives: This section lays out more specifically what progress would address the sites needs. It should (to the extent possible) state the actual objectives rather than state a specific type of technology (e.g., "reduce and manage tritium impacts" rather "than tritium separation methods"). Bounds would be the things that would tell the potential PIs what they need to do to distinguish their work from known/available technology.

Figure 7. Annotated outline of the technical targets. 
While the general outline of the targets was consistent, each target exhibits a unique character. For example, the target related to organic source zones advocates continuing the past SCFA progress in moving through the organic source zone challenges in an orderly and technically based manner. It recognizes and summarizes the successful DOE-interagency historical development efforts that have resulted in several alternative technologies (now commercially available) for cleaning up organics in simple to moderately complex settings (steam/DUS, six phase heating, and surfactants). These successes resulted from collaborative investment by DOE and other federal agencies. The specific focus for future activities is on enhanced removal and in-situ destruction technologies for conditions that are more difficult than those already addressed by SCFA (fractured systems and low permeability media). Thus, the target recommends a central strategy of collaboration with other agencies to advance practical application of organic source zone treatment methods. Similar to the example above, promising technical directions and themes were identified for each target, and these were captured in the detailed write up.

\section{Results and Recommendations}

A few broad themes emerged in several of the targets. These centered on ideas such as, "using holistic approach to solving the problems expressed in the needs" and "selecting actions that work with, rather than against, nature." A good example of this is the target on Integrated Storage and Containment Concepts. This particular target identifies that the "dry tomb" concept that is at the heart of current containment objectives/regulations precludes important technical solutions. Containment would be enhanced in a uniquely valuable manner by, when possible, incorporating treatment in the barrier design to detoxify and/or immobilize contaminants. The resulting holistic approach is essential to future success for DOE sites. A "dry tomb" needs to provide permanent isolation (it works against nature) and permanent commitment to monitoring/verification, while a smart barrier needs to provide protection and verification for a limited time (until the waste no longer poses a hazard). The general concept of minimizing negative collateral damage associated with actions was highlighted in several targets along with specific related technical issues and recommendations that SCFA could pursue to strategically assist its end users. Each of the targets and its unique theme(s) are provided in the executive summary, a one page synopsis for each target is provided in Appendix A, and the complete texts are provided in Appendix B.

In the final session at the inaugural meeting and during the follow-up meeting, the meeting participants assessed the process and developed recommendations. There was consensus that the strategic targeting exercise was useful and that the draft targets contain very constructive information. The draft targets are suitable and important input to a strategy to modify the technical basis of the SCFA program. In particular, the group developed the following "Technical Target" related recommendations for SCFA:

- Summary level information should be developed to assist SCFA in conveying the targets to DOE-HQ and to site end users. (this report) 
- Targets should be used, in modified form and in cooperation with the end users, to develop calls for proposals.

- A technical program plan, based on the targets, should be developed to support the Strategic Plan. The Strategic Plan needs to be modified to incorporate the technical target concept and to outline the process to develop the targets.

- Targets should not immediately replace work packages because the existing work package ranking system would allocate funding among them based on raw needs counting and without regard to a strategic portfolio. Instead, the group felt that the existing work packages should be refined and changed in a manner consistent with the targets to allow broadly funding the most important themes across all targets. This recognizes that the targets are already a subset of a more comprehensive list of possible activities and that "cherry-picking" from the subset may leave a serious enduser problem unanswered and foreclose on a holistic solution

- Because efforts are already underway to solicit proposals and fund new projects soon, the group recommends that the proposals should be reviewed now against the draft targets to ensure that new projects align with the strategic direction and do not lock up funding that is needed to implement the Technical Targets. The targets should also be used as a tool during midyear reviews and other SCFA assessment activities.

- The target development team should reconvene to refine and finalize the Technical Targets and to develop a stronger technical basis for the identified themes. An approach to prioritize the targets and the themes within the targets and recommendations to SCFA on how the work packages could be restructured were also items that were of interest to the team. (second Golden meeting in November)

- Consider bringing in subject matter experts (e.g., principal investigators working in the areas addressed by the strategic targets to help refine them). Participation in the Technical Target Team should be examined to assure participation by appropriate representatives from other agencies and national scientific academies.

- $A$ key to the approach was selecting appropriate participants and limiting the group to a size that was suited to the interactive rapid-response triage-style brainstorming and writing process. This process was viewed as a success by the participants and should be refined and continued.

- Sub-targets should be expanded and mapped with EMSP projects, ASTD projects, NETL projects, NABIR projects, SERDP/ESTCP projects etc.

- Consideration should be given to an effort in which technical experts would interrogate the technical baselines at each site periodically (e.g., a 3-year cycle) to help SCFA fully understand the sites' problems (versus relying on STCG needs). This process would enable SCFA to help the sites identify potential cost savings, introduce $R \& D$ efforts into the baseline, and refine the SCFA technical program plan. This kind of approach could be part of, and represent a refinement of, the SCFA needs assessment process. 
Communication of the targets, both within DOE and to outside organizations was highlighted in the recommendations. The group discussed the organizations that need to hear about the Technical Targets and how this communication should be done. The following organizations were identified for this communications and feedback effort:

- SCFA and allied programs (CMST, ITRD, TechCon, EMSP, IP, UP, etc.)

- DOE-HQ

- DOE site end users

- Strategic Lab Council, EM Core Labs, EM Core Team

- Other DOE programs

- EPA

- DOD including ESTCP and SERDP

- Others within the Federal Remediation Roundtable

- USGS

- EMAB

- The scientific community

- National Academy of Sciences

- Principal investigators (current and prospective)

- ITRC

- RTDF

- Technology providers (vendors and consultants)

- Stakeholders (e.g., citizens advisory boards)

With respect to how to share the targets, the group suggested using the existing SCFA and EM communication channels. In doing so, it will be important to ensure understanding that the focus of the targets is on DOE-EM sites. The focus of the communications should be on improving the understanding of the SCFA technical program instead of soliciting comments, but should provide an iterative process with feedback to the targets. Once the targets have been refined, consideration should be given to placing information about them on the SCFA website. Other suggestions included (1) doing one or more workshops with potential or selected principal investigators (e.g., like the Hanford Applied Science Workshop), (2) using the upcoming Bechtel five-sites meeting to share the draft targets, and (3) conveying the targets during the SCFA needs assessment process with DOE site end users. 


\section{Appendix A}

\section{One Page "Study Guide Style" Summaries of SCFA Technical Targets Developed in Golden CO}

\section{Ensuring Environmental Stewardship}

- Improving the Technical Basis for Setting Remediation Goals........... A1

- Methods to Verify and Validate Performance......................... A2

\section{Eliminating Contamiñānt Sources}

- Organic Source Zone Stabilization and Treatment..................... A3

- Metals and Radionuclide Source Zone Stabilization and Treatment...... A4

\section{Isolating Contaminants}

- Advanced Sustainable Containment Systems.......................... A5

- Integrated Containment-Treatment Concepts - "Smart Containment".... . A6

\section{Controlling Contaminant Plumes}

- Effective and Sustainable Technological Solutions for Contaminant Plumes

- Tritium Management and Risk Reduction.

\section{Enabling DOE's Clean-Up Efforts}

- Subsurface Access and Delivery

- Techniques and Technologies that Support Characterization

- Biogeochemical Processes that Determine Contaminant Fate...............

- Strongly Heterogeneous Systems (such as fractured rock)

- Advanced Environmental Modeling. 
Study Guide Style Quick Summary of SCFA Technical Target

\section{Improving the Technical Basis for Setting Remediation Goals}

Strategic Investment Category: Ensuring Environmental Stewardship

Overview: This target recommends working with end users, regulators and stakeholders to develop a more holistic approach for setting goals. This target identifies several specific technical advances that would improve decisionmaking and recommends rapidly integrating the advances into decision support resources for beneficial use in baseline activities. Vital development themes with particular promise to DOE include: 1) prioritizing the desirability of end states, 2) time phased decisionmaking, 3) technically defensible assessment of collateral damage, and 4) accounting for baseline risk for nâtural elements and synergistic effect in contaminant mixtures.

Comments: This target addresses vital technical aspects of this important policy topic. One of the most interesting topics to the technical target development team was the identification of collateral damage as an important element to incorporate into decisionmaking (does the cleanup action actually do more harm than good - by using excessive energy, by eliminating habitat, etc.). An area that may be of particular interest to DOE and end users over the next several years is how to deal with remediation of naturally occurring substances such as arsenic or mercury. The target is strong in its description of what is needed to support improved decisionmaking, but points out that the ultimate decisions in this area are policy rather that technical. This has been the reason that these topics are not traditionally viewed as desirable to tackle in a scientific and technical R\&D program.

Importance: In almost every case, excessive costs and failures of cleanup systems can be traced back to poor goal setting.

What the target does not say: The target does not advocate continuing a cookbook style use of standard risk assessment approaches. Rather, it suggests specific steps for providing a technical basis to alter the standard approach and incorporate balancing factors in decisionmaking.

Important comments from team during review: Reviewers felt that this was an important and challenging target.

Links to other technical targets: Depends on key progress in "Advanced Environmental Modeling" and "Methods to Validate and Verify Performance". 


\section{Methods to Verify and Validate Performance}

Strategic Investment Category: Ensuring Environmental Stewardship

Overview: This target recognizes the need to develop distinctly different technologies for short term performance monitoring versus long term monitoring. The former relies primarily on improved sensor development, emplacement and data integration, while the later relies primarily on developing wholly new verification paradigms to allow monitoring for decades or centuries. Large scale methods (remote sensing, geophysics), integrating methods (flux or release measurements), and indicator/surrogate methods, among others appear promising for long term monitoring.

Comments: This target addresses an active and important topic area. In its original form, the target was strong in its description of what is needed to support short-term verification and validation of system performance. In its revision the team focused on long term monitoring methods emphasizing their ultimate importance and value, and highlighting in moderate detail what is needed - and what is not needed - for long term monitoring.

Importance: Field experience indicates that overall environmental management costs (and even the regulatory approval to proceed with cleanup activities) are primarily determined by verification and validation issues/approaches/costs.

What the target does not say: The target does not advocate the most popular long term monitoring paradigm of numerous sensors, dataloggers (or wireless transmitters) and "real time" data as the central concept for long term monitoring. Some of these features are retained in the description of short-term performance verification, however. The target does identify and advocate alternative paradigms for long term monitoring that should provide robust and defensible monitoring for less cost and in a way that is useful to EM and to stakeholders. Such monitoring should be considered one of the multiple defenses recommended for environmental response activities by the National Academy of Sciences in their Long Term Stewardship report(s).

Important comments from team during initial review: Reviewers felt that this was an important target that addressed most of the important issues in an appropriate manner.

Links to other Technical Targets: Depends on making key advances in fate and transport modeling as described in "Advanced Environmental Modeling" and in developing robust and reliable sensors for key contaminants as described in "Techniques and Technologies that support Characterization". 
Study Guide Style Quick Summary of SCFA Technical Target

\section{Organic Source Zone Stabilization and Treatment}

Strategic Investment Category: Eliminating Contaminant Sources

Overview: This target advocates continuing the past SCFA progress in moving through the National Academy of Sciences' chart of remediation challenges from less difficult to more difficult. Thus, the target recommends a central strategy of collaboration with other agencies to advance practical application of organic source zone treatment methods. The specific focus for future activities is on enhanced removal and in-situ destruction technologies for conditions that are more difficult than those already addressed by SCFA (fractured systems and low permeability media). Another vital objective highlighted in this target is development and selection of techniques that are compatible with a transition to less aggressive and more passive polishing of the site - for example, technologies that do not foreclose on biological remediation because they cause long term sterilization of the subsurface. A final concept described in the target is the need for characterization tools to designate and delineate the source zone so that expensive and aggressive cleanup methods are not applied in areas where they are not needed.

Comments: A key strength of this target is that it recommends addressing "organic source zone" technology challenges in an orderly and technically based manner. It recognizes and summarizes the successful DOE-interagency historical development efforts that have resulted in several alternative technologies (now commercially available) for cleaning up organics in simple to moderately complex settings (steam/DUS, six phase heating, and surfactants). These successes resulted from collaborative investment by DOE and other federal agencies.

Importance: Successfully remediating organic source zones is an absolute requirement for implementing successful cleanup strategies in the downgradient primary and dilute fringe portions of the plume. Slow "leakage" from the source zone is the reason that plume treatment technologies are perceived to be failing (taking hundreds of years).

What the target does not say: The target discourages the concept that physical containment is viable for organic source zones.

Important comments from team during initial review: This was positively received.

Links to other Technical Targets: Depends on making key advances in fate and transport modeling as described in "Advanced Environmental Modeling" and in developing efficient "Access and Delivery" methods. This target also relies on developing and implementing improved decisionmaking and performance verification approaches as described in "Methods to Verify and Validate Performance" and "Improving the Technical Basis for Setting Remediation Goals". 
Study Guide Style Quick Summary of SCFA Technical Target

\title{
Metals and Radionuclide Source Zone Stabilization and Treatment
}

\author{
Strategic Investment Category: Eliminating Contaminant Sources
}

Overview: The focus of this target is on treatment/removal approaches and techniques that overcome the issues of complex waste near the source and facilitate transition to more traditional (i.e., lower cost) techniques and/or to document that residual contamination is stabilized to protect human health and the environment.

Comments: This target is quite different than the one related to organic source zones because of the fundamentally different nature of radionuclides and metals. In this case, technology development leading to stabilization is emphasized for metals/radionuclides where transport is slow and exceedances of standards (e.g., groundwater concentrations) are relatively low. Important, but exceptional, cases where geochemistry is highly perturbed (e.g., high level waste tanks at Hanford, uranium in groundwater at Oak Ridge) are important and issues of understanding and manipulating chemical speciation are a vital theme for such sites. The target recognizes that physical containment is a viable option for relatively small source zones.

Importance: Several of the highest visibility recent DOE environmental stewardship challenges are related to metals and radionuclides source areas. The "surprise" of finding cesium "deep" in the Hanford vadose zone or "unexpectedly" measuring plutonium in monitoring wells at the Nevada Test Site highlights the importance of this topic.

What the target does not say: The target does not suggest that the science base will be in place in a foreseeable period to eliminate all surprises, but rather it recommends that efforts be directed at understanding and manipulating key variables to reduce uncertainty, increase credibility, and to allow responsible corrective actions to be implemented.

Important comments from team during initial review: There was general consensus that the main points raised in the target were appropriate and the reviewers felt that it is an important topic. The themes in this target are not well suited to addressing the distinct issue of low concentration - large area - surface contamination (e.g., Nevada Test Site, etc.).

Links to other Technical Targets: Depends on making key advances in fate and transport modeling as described in "Advanced Environmental Modeling" and in developing efficient "Access and Delivery" methods. This target also relies on developing and implementing improved decisionmaking and performance verification approaches as described in "Methods to Verify and Validate Performance" and "Improving the Technical Basis for Setting Remediation Goals". 
WSRC-RP-2002-00077

Revision 0

Page A5 of A13

Study Guide Style Quick Summary of SCFA Technical Target

\section{Advanced Sustainable Containment Systems}

Strategic Investment Category: Isolating Contaminants

Overview: Devise/Develop containment systems based on natural analogs. Develop containment systems that have robustness based on fundamental theoretical processes ("long life") and which integrate with related remediation activities.

Comments: This target was quite well conceived and written and carefully and comprehensively lays out the vital objectives related to science, engineering/implementation, and creative monitoring. The emphasis on natural analogs as a means to document the long term performance of containment systems was interesting, useful and strategic.

Importance: Properly applied and monitored, physical containment and barriers will remain a central activity in DOE environmental management for the foreseeable future. Advancing the science and technology base relatively rapidly is particularly important to closure sites that need to implement and document such systems in the next several years.

What the target does not say: The target does not advocate barriers as a sole solution to environmental problems. Instead, it suggests that they be considered one of the multiple defenses recommended for environmental response activities by the National Academy of Sciences in their Long Term Stewardship report(s).

Important comments from team during initial review: Reviewers felt that this was one of the most complete and well-written targets. It is relatively comprehensive (i.e., it could comprise an entire reasonably anticipated research program budget) and will require some prioritization. A few reviewers commented that this target had significant overlap with aspects of the "Integrated Containment-Treatment (Smart Containment)" and that they might be combined. Others felt that the more pragmatic objectives of cap verification - items that are currently critical to closure sites - might be de-emphasized if the targets were combined.

Links to other Technical Targets: Depends on making key advances in fate and transport modeling as described in "Advanced Environmental Modeling" and in developing and implementing improved decisionmaking and performance verification approaches as described in "Methods to Verify and Validate Performance" and "Improving the Technical Basis for Setting Remediation Goals". 
Study Guide Style Quick Summary of SCFA Technical Target

\section{Integrated Containment-Treatment Concepts - "Smart Containment"}

Strategic Investment Category: Isolating Contaminants

Overview: Develop integrated systems so that solid hazardous and/or radioactive waste is stabilized or detoxified during a period of containment or isolation. To support such a strategic development, potential treatment processes (delivery systems and their compatibility with the isolation systems) must be evaluated, as well as methods to monitor treatment progress and document when the waste containment is no longer needed.

Comments: This target is a specific example of a technical approach that crosses traditional administrative boundaries and which implements the NAS/NRC recommendation of building multiple defenses into long term stewardship systems. The technical target team felt that this type of target had a unique character that may encourage a crosscutting strategic investment and may be a desirable style of target to consider for future development.

Importance: The ability to integrate and view actions more holistically and to cross administrative boundaries in developing and implementing solutions represents one of the most promising areas for SCFA to make technical contributions.

What the target does not say: The target does not support the implementation of traditional (RCRA style) dry tomb containment systems that are technically fragile for most situations.

Important comments from team during initial review: Reviewers felt that this target was well conceived.

Links to other Technical Targets: Depends primarily on making key advances in developing and implementing improved decisionmaking and performance verification approaches as described in "Methods to Verify and Validate Performance" and "Improving the Technical Basis for Setting Remediation Goals". 
WSRC-RP-2002-00077

Revision 0

Page A7 of A13

Study Guide Style Quick Summary of SCFA Technical Target

\section{Effective and Sustainable Technological Solutions for Contaminant Plumes}

Strategic Investment Category: Controlling Contaminant Plumes

Overview: Several vital scientific and technical objectives remain: 1) optimizing active treatment systems, 2) understanding the relationship between performance and the completeness of source zone treatment, 3 ) developing technologies that transition more quickly to monitored natural attenuation (e.g., through emplacement of long term reagent materials, through identification of measurable natural processes that destroy, stabilize, or detoxify dilute plume contaminants), and 4) developing design approaches and viable monitoring strategies. The target does not distinguish between organics, metals, and radionuclides, because the possible actions and challenges for the various contaminants tend to come together in the primary plume and more dilute areas. This target is structured to support development of the general class of technologies often referred to as "monitored natural attenuation." The target emphasizes that sustainable technologies must be technically based to be accepted and implemented. The target was strong in the way that it linked mechanisms and monitoring concepts.

Comments: This target was strong in the way that it linked source zone treatment with transitioning to monitored natural/sustainable processes. In general, active treatment methods for the primary plume are relatively mature, while transitioning approaches and paradigms require significant development.

Importance: A unified and linked concept in remediation that addresses the source primary plume - and distal dilute plume in an organized and comprehensive fashion represents the most promising approach to more rational action at many DOE facilities. Development, documentation, and implementation of sustainable technologies has been identified as one of the most important medium to long term goals in DOE EM. This topic is at the heart of NAS/NRC recommendations on Long Term Stewardship and these "technologies" represent one of the final protections in the recommended multiple defenses.

What the target does not say: The target does not say that pump and treat does not work rather that a treatment train or systems approach is needed to address the various portions of the plume with their varying characteristics. The target highlights the fact that rational actions in the primary plume and dilute zone are predicated on source zone stabilization and treatment. The target does not say that sustainable technologies (e.g., MNA) are desirable and applicable to every site or that such technologies can always be implemented alone. Rather, the target emphasizes incorporating this class of action into a treatment system.

Important comments from team during initial review: Reviewers felt that this target was well conceived. 


\section{Effective and Sustainable Technological Solutions for Contaminant Plumes (cont)}

Links to other Technical Targets: Depends on making key advances in "Advanced Environmental Modeling" and in "Methods to Verify and Validate Performance" and "Improving the Technical Basis for Setting Remediation Goals". This target also depends on success in eliminating both the organic and metal/radionuclide source zones that feed contaminant plumes.

\section{Tritium Management and Risk Reduction}

Strategic Investment Category: Controlling Contaminant Plumès

Overview: Tritium is a contaminant with unique challenges. The vital objectives for this target include: 1) "cost effective" tritium treatment technologies, 2) alternate tritium remediation strategies to reduce releases, exposure and risk, and 3) tritium characterization and monitoring tools, with particular emphasis on identifying plume arrival or tritium fluxes and risk. The target defines guidelines for determining what is meant by "cost effective" to assist in evaluating if a proposed technology represents an advance over the baseline. Interestingly, the recommended focus of the target away from traditional "treatment" can be seen in many of the recent tritium related EM projects (cited in the target). The objectives in the target would directly support these activities.

Comments: The most important contribution of this target is shifting the emphasis away from tritium (hydrogen isotope) separation and toward tritium management and risk reduction. Remote monitoring of tritium in deep wells as a surrogate to delineate the edge of the contaminant plume was viewed as an important topic and an opportunity to rapidly demonstrate in the real world some key concepts within the characterization and verfication/validation targets.

Importance: Tritium treatment and monitoring were identified by several DOE sites across the country. The primary importance of this target, however, is providing detailed information to help SCFA identify what is not needed versus what might represent substantive contributions to end users.

What the target does not say: The target does not say that the primary solution to tritium at DOE sites is traditional "treatment."

Important comments from team during initial review: Reviewers felt that this target was well conceived.

Links to other Technical Targets: Depends on making key advances in "Methods to Verify and Validate Performance" and "Improving the Technical Basis for Setting Remediation Goals". 
Study Guide Style Quick Summary of SCFA Technical Target

\section{Subsurface Access and Delivery}

Strategic Investment Category: Enabling DOE's Clean-Up Efforts

Overview: The most important objectives highlighted in this cross cutting target are: 1) access under obstructions, 2) delivery of fluid for treatment or containment, 3) deep access, 4) difficult access, and 5) delivery of devices.

Comments: This target was well conceived and written. The writers addressed access for sampling, delivery of devices, and delivery of fluids for subsurface manipulation and remediation. The target identifies that poor access, rather than poorly understood chemistry or biology, is the primary reason for poor remediation system performance. The target documents the strong historical contribution of DOE (horizontal wells, cone penetromenter systems, and the like). Because of the wide range of end-user needs on this topic, the target provides an initial assessment of priorities and tabulates the relative importance of the various vital objectives that were identified.

Importance: Improved access methods were a widely distributed and critical end-user need throughout the DOE complex. This cross-cutting area has not been emphasized over the past several years and the group felt that a target to focus strategic investment was absolutely necessary. This target is particularly important to closure sites that need to implement and document cleanup in the next several years.

What the target does not say: The target does not suggest that improving access, without appropriate decisionmaking, technology selection and verification methods will entirely solve key environmental challenges at DOE sites.

Important comments from team during initial review: Reviewers felt that this was a complete and well-written target. Comments were particularly positive on the approach used for providing insight and prioritization of the five vital development objectives.

Links to other Technical Targets: Depends on making key advances in performance verification approaches as described in "Methods to Verify and Validate Performance". This target supports the cleanup targets, particularly those related to source zone treatment and stabilization. 
Study Guide Style Quick Summary of SCFA Technical Target

\section{Techniques and Technologies that Support Characterization}

Strategic Investment Category: Enabling DOE's Clean-Up Efforts

Overview: Vital themes identified and described for this target include: 1) improvement of non-invasive characterization technologies, 2) measurements at various scales (point and volume integrated) to support multiple objectives, 3) development of field deployable systems, and 4) integration of multiple types of characterization data.

Comments: This was a complex and difficult target to write. The target does a good job in identifying the important issues, but needs more detail and prioritization to help it to be strategic (see review comments). One of the most acclaimed aspects of the target is a discussion of moving toward field screening to enhance representativeness and to reduce costs and, where needed, to perform measurements in-situ in cases where representative measurements can not be made at the surface. Scale issues are well described, with related vital objectives providing solutions from various complementary perspectives (integrating measurements, direct push "continuous" measurements, etc.).

Importance: Characterization currently represents the largest early project investment. As a result, significant improvements in characterization methods will rapidly improve the EM program.

What the target does not say: The target does not say in-situ measurements are needed for all constituents.

Important comments from team during initial review: Reviewers felt that this target was generally well conceived but probably needed more detail and prioritization on strategic investment directions (parts of it read more like a comprehensive list of an ideal program).

Links to other Technical Targets: Depends on creatively exploiting observations and data from basic science studies and on making key advances in performance verification approaches as described in "Methods to Verify and Validate Performance". This target supports the cleanup targets, particularly those related to source zone treatment and stabilization. 
Study Guide Style Quick Summary of SCFA Technical Target

\title{
Biogeochemical Processes that Determine Contaminant Fate
}

\author{
Strategic Investment Category: Enabling DOE's Clean-Up Efforts
}

Overview: The effective implementation of remediation strategies and natural attenuation for the cleanup of DOE sites depends on understanding critical chemical, physical and biological processes. Particular important research themes include: 1) redox conditions that affect biogeochemical processes, 2) anthropogenic influences on the biogeochemistry of natural systems and extreme environments, and 3) coupling and scaling issues.

Comments: This target was strong because it prioritized the complex topic and clearly identified the key/vital early investment themes embodied in end-user experiences. What makes this topic challenging is that there has been so much work done in the area and there are so many uncertainties in specific details, and that there are so many scientists who advocate investing in their specific area of interest. Thus, this subteam made a relatively courageous decision to highlight a few key areas and open the target up to the potential criticisms that this topic, or that topic, is overlooked. The consensus after the target was written, was that the highlighted items were appropriate and that they represent strategic technical objectives that would advance both the critical science and be useful.

Importance: The particular biogeochemical processes highlighted in this target are the ones responsible for most of the biogeochemical end-user problems (and/or they represent potential creative solutions via subsurface manipulation).

What the target does not say: The target does not provide a comprehensive prioritized list of all of the biogeochemical uncertainties nor a detailed plan to resolve them all over a 10 or 20 year period (as is done in the Vadose Zone Roadmap for example). The more comprehensive look, while highly desirable, was viewed as unrealistic and not aligned with the needs of targets to provide strategic investment information to SCFA.

Important comments from team during initial review: Reviewers felt that this was an important, challenging and well-written target.

Links to other Technical Targets: Requires continued basic research and approaches to link the results to the field scale through the "Advanced Environmental Modeling" target. 


\section{Strongly Heterogeneous Systems (such as fractured rock)}

\section{Strategic Investment Category: Enabling DOE's Clean-Up Efforts}

Overview: This target emphasizes the significance of the subject issue and the challenge and the significant historical investment and research. Based on the state of science and art, there is a vital need to develop alternate, non-deterministic approaches (similar to weather modeling) as a critical path in the future.

Comments: This was one of the most interesting and challenging targets to write. It was strategic and particularly courageous in its vital objectives because it directly challenges the status quo (both in terms of historical investment and in terms of pockets of scientific support). In a manner analogous to the tritium target, the value of this target may be in what is does not recommend rather than what is does recommend. In particular, this target does not recommend bigger computers or more deterministic modeling as central solutions to strongly heterogeneous systems!

Importance: Strongly heterogeneous systems were identified as critical needs at several DOE sites:

What the target does not say: The target does not recommend many of the popular approaches commonly considered central to solving problems associated with this topic (at least for SCFA investment).

Important comments from team during initial review: Reviewers felt that this target was generally well conceived, strategic, focused and well-written.

Links to other Technical Targets: Requires rather specialized objectives being addressed within the "Advanced Environmental Modeling target" and will support those sites where strongly heterogeneous systems are a critical barrier to progress. 
Study Guide Style Quick Summary of SCFA Technical Target

\section{Advanced Environmental Modeling}

\section{Strategic Investment Category: Enabling DOE's Cleanup Efforts}

Overview: The vital needs associated with modeling fundamental environmental processes are: 1) identifying and filling fundamental environmental process knowledge gaps (as described in the Vadose Zone Roadmap, for example), 2) improving uncertainty quantification techniques, and 3) near term transitioning techniques from state-of-art to state-of-practice.

Comments: This was a complex and difficult target to write. The target does a good job in identifying the important issues. In the second meeting more detail and prioritization was added to help it to be strategic (see review comments). Some of the target's original objectives were too general ("Identification and filling of fundamental environmental process knowledge gaps", "Development of more comprehensive uncertainty quantification techniques"). Others represent good near term goals ("Transitioning from state-of-art computing to state-of-practice in modeling").

Importance: The ability to accurately model and/or understand fundamental processes is often identified as critical success. There is a perception that better models will "eliminate" surprises and lead to optimized remediation.

What the target does not say: The target does not completely support or refute ideas about the role of deterministic modeling in addressing this target or improving end-user actions.

Important comments from team during initial review: Reviewers felt that this target needed more detail and focus on a subset of critical issues, possibly using biogeochemical processes as a model for how to select items to include. This modification was made during the second meeting.

Links to other Technical Targets: Progress toward this target is the most commonly cited link to meet almost all of the other technical targets. This target requires information from the biogeochemistry, heterogeneous system, and other science providing targets. 


\section{Appendix B}

\section{Full Texts of the SCFA Technical Targets Developed in Golden CO}

\section{Ensuring Environmental Stewardship}

- Improving the Technical Basis for Setting Remediation Goals........... B1

- Methods to Verify and Validate Performance.......................... B6

\section{Eliminating Contaminant Sources}

- Organic Source Zone Stabilization and Treatment..................... B B 12

- Metals and Radionuclide Source Zone Stabilization and Treatment...... $\quad$ B18

\section{Isolating Contaminants}

- Advanced Sustainable Containment Systems........................ B22

- Integrated Containment-Treatment Concepts - "Smart Containment"... $\quad$ B27

\section{Controlling Contaminant Plumes}

- Effective and Sustainable Technological Solutions for Contaminant Plumes

- Tritium Management and Risk Reduction............................ B B

\section{Enabling DOE's Clean-Up Efforts}

- Subsurface Access and Delivery ....................................... B41

- Techniques and Technologies that Support Characterization.............. B B B

- Biogeochemical Processes that Determine Contaminant Fate............. B B

- Strongly Heterogeneous Systems (such as fractured rock)................. B51

- Advanced Environmental Modeling .................................. B54 


\section{Improving the Technical Basis for Setting Remediation Goals}

Team: Brian Looney, James Murphy, Norine Noonan, Jim Helt, Tyler Gilmore, Mike Powell, Tom Ivory and Doug Burns

\section{Summary of Need(s):}

- Need to use more holistic approaches to site remediation decisions and to integrate . these approaches into decision support resources. The four themes of this target are identified by italics below.

- Need general improvements in EM decision processes

- Need to define desirable end states

- Need to emphasize time sequencing of decisions and flexibility for future options in the face of significant technical uncertainties.

- Need to add elements not adequately considered in current decision processes

- Need to understand "collateral damage" of actions and incorporate these considerations more explicitly in decision framework.

- Need to use approaches that consider the presence of background levels of toxic or radioactive elements and synergistic effects of mixtures of contaminants.

\section{Relevance:}

Strengthening the decision process for remedial actions will improve the outcomes and potentially lower costs for long-term stewardship of DOE sites. Initially defining desirable end states for sites, preserving future options for remediation decisions, and minimizing collateral adverse effects (either to ecological health, human health or both) all contribute to decision processes that are better for the environment and more robust. In addition, the combination of these elements with tools and approaches that emphasize the certainties for nearer-term actions while increasing understanding of uncertainties with the most influence on future actions can enable site owners and public stakeholders to have greater confidence in the overall decision pathway and ultimate outcomes at the various sites. Methods and tools that consider the presence of background levels of naturally occurring toxic or radioactive elements can add an important balance to decisions, with a concomitant positive impact on the cost of remediation.

\section{Status:}

Typically, DOE environmental management decisions are based on the computed severity of potential harmful impact of hazardous materials to public health, the environment, and to the workers involved in cleanup operations. This risk assessment process can be technically challenging and the results ambiguous and controversial. The DOE Strategic Plan cites the first objective of the EQ business line to reduce the most serious risks from the environmental legacy of the U.S. nuclear weapons complex. However, the current scientific uncertainty in the consequences of exposure to environmental levels of radiation and chemical contamination raises questions about the ability to make credible policy decisions on a risk basis. These uncertainties also contribute to problems in the communication of risk to the public. 
A recent, independent review of the use of risk by DOE, "Peer Review of the U.S. Department of Energy's Use of Risk in its Prioritization Process" (Peer Review Committee of the Consortium for Risk Evaluation with Stakeholder Participation, Dec. $15,1999)$, cited several areas where advances in knowledge and methodology would support the use of risk in decisionmaking. Among those areas are the use of a more holistic and integrated approach to risk assessment, understanding the "collateral damage" of remedial actions (impacts on worker health, risks of chemical hazards, transportation and ecological risks), and more transparent risk assessments methods that can achieve greater credibility with stakeholder audiences. DOE has made significant progress in these areas. Notable examples are the widely used DOE Center for Risk Excellence products - a public database of risk based decisions, approaches for estimating social and cultural impacts, and the like. Despite these advancements, additional technical progress is crucial over the next few years to properly focus and optimize the large DOE cleanup effort.

Risk based decisions for sites that contain naturally occurring toxic and radioactive constituents are performed across the DOE complex and typically lead to ambiguous assessments. Background studies to define the average concentration and variation in many of the important elements have been performed at several DOE sites (Hanford, Oak Ridge, Savannah River Site, and others), and have been published by USGS. More limited studies for specific environments are available in the scientific literature. In some decision and risk evaluations, these backgrounds are used as one of the possible "ARARs", but the actual calculation of risks and interpretation of results to select an option is typically performed without consideration of background. Emerging measurement approaches (e.g., sensitive toxicological indicators, genetic effects) are currently being used to set unprecedented compliance standards near background levels for a variety of elements such as $\mathrm{Hg}$ and As.

All of the Vital Scientific and Technical Objectives discussed below are being pursued in some form or are being considered today. But there is not a consistent consideration of their impacts, nor are there widely accepted tools and methods that are used to achieve the objectives.

\section{Vital Scientific and Technical Objectives:}

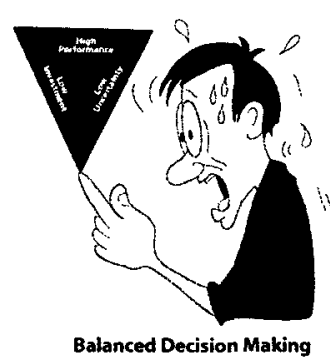

Decisionmaking is the methodology used to consider all the important factors in choosing an alternative. The objective of holistic decisionmaking is to broaden the goals and criteria to consider where specific improvements are possible that will increase the quality of a decision. These improvements will affect both the considered alternatives and the discriminators used to judge the tradeoffs among the alternatives. There are both near-term (next 5 years) and $\mathrm{mid} /$ long-term (beyond 5 years) improvements considered. Several of these improvements will require collaboration between DOE and regulators to increase the likelihood of success in integrating these improvements into the decision process 
Improved approaches for defining and selecting desirable end states are critical to DOE's environmental stewardship activities. Cost effectiveness, public acceptance, and ultimate success of stewardship activities require improved decision processes that include the development of approaches to prioritize the desirability (not just acceptability) of alternative end states. Selecting an end state that is "highly desirable" to the public (e.g., an ecological preserve) may lead to significant reductions in required actions and costs. Such an approach and descriptions of the possible benefits have been recommended in recent public policy documents (e.g., "Wasteland to Wilderness"). In general, prioritizing end state desirability alternativesis the explicit technical implementation of the long-term strategy of DOE to address the legacy from nuclear production operations. This objective would also support land use planning commitments and assist in balancing environmental considerations, brownfield options (industrial/nuclear production), and greenfield options (unrestricted use). The principal benefit of prioritizing end-state desirability and selecting from those alternatives that are most desirable is that it allows DOE and its stakeholders to work toward a shared goal. Existence of a shared goal will reduce the pressure to resolve all technical uncertainties because all parties want to facilitate progress.

\section{Time-Phased Decisionmaking}

Time-phased decisionmaking is a concept that acknowledges the need to make some decisions now, followed by uncertainty resolution and adjustment. The concept encapsulates a "learn-decide-learn-decide" sequence. The "decide" portion is based on selecting a desirable alternative (one that does not preclude a promising future action) and the "learn" portion of the cycle focuses on uncertainty resolution to enable the next decision. Without the phased decision process, the near-term alternatives often attempt to solve an entire problem without a full understanding of the consequences. Instead, partial solutions could be implemented, with future actions at a later date when more knowledge or better alternatives are available. Methods and protocols are needed to bring about the alternatives identification and the discriminator evaluation to include the flexibility criterion. Methods and tools are also needed to perform impact analyses of the uncertainties that can be integrated with the uncertainty resolution process and the time-phased decision methods and protocols. All these analyses methods, protocols, and tools need to be seamlessly integrated into the decision process. Focus on high impact uncertainties can lead to more effective resolution by the appropriate discipline areas (e.g., using a roadmapping plan) and leads to faster and better future decisions. Adding functionality and data to modeling to predict affects of current actions on potential future actions improves the understanding of near-term decisions on longterm alternatives. 
Collateral damage associated with environmental clean up actions are currently not quantified or considered in most decision and selection processes. Importantly, traditional analysis does not routinely consider the important collateral damages that are often associated with implementing a clean-up action - e.g., energy use, loss of habitat, natural resource use,

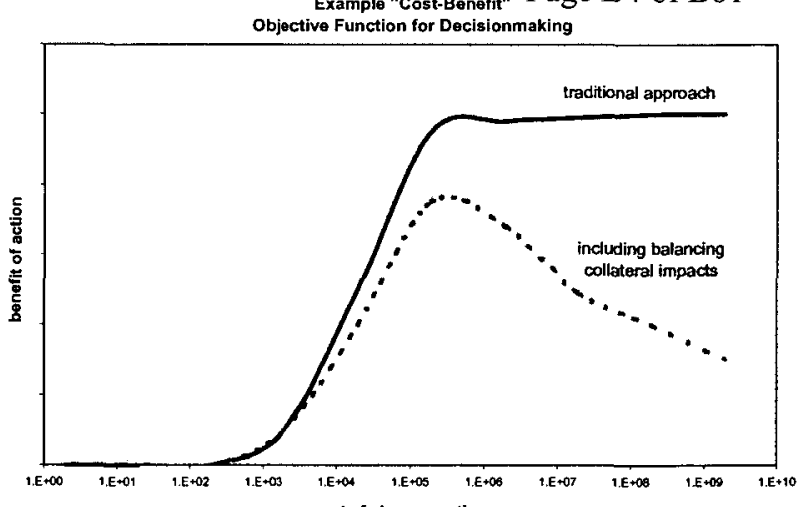
occupational risk, secondary waste. Most decisions are based simply on comparing calculated human health risks and ecological impacts for the various options and assessing the overall result in terms of maximizing benefit (or minimizing risk). In this traditional approach, increased costs and complexity in the assumed cleanup generally increase benefits - with significant benefit in the low to intermediate cost range and generally less benefit at high expenditures (see simplified diagram.) As depicted on the diagram, collateral damages typically increase for more complex and expensive clean-up actions and reduce the net benefit even as costs are increasing. Thus, these impacts, if incorporated into remediation decisions, provide balance and more clearly define the optimum decision at a lower cost. The principal technical challenge in meeting this vital objective is developing technically defensible protocols to quantifying and incorporating collateral damage into decisionmaking processes. Recent advances in the evaluation of energy production and quantification of global environmental impacts may provide information for pilot studies, with other major collateral impacts prioritized and evaluated as justified. Technically defensible protocols for defining and assessing collateral damage and incorporating these protocols into the environmental decision process for sites are needed and represent a significant opportunity to improve DOE's cleanup mission.

\section{Considering Background Levels and Synergistic Effects}

There is a vital need to develop technical approaches to set cleanup goals and support environmental decisions for situations that are not properly addressed by the current simplified additive-linear risk assessment calculation. Two particular cases relate to naturally occurring toxic and radioactive elements and nonlinear impacts due to interactions among the components of a contaminant mixture. The "background issue" is acute for elements that are toxic at levels near natural baseline levels (such as $\mathrm{Hg}$ ) and for elements that can exist at high concentrations/activities in certain natural settings (such as $\mathrm{As}, \mathrm{Pb}$, and uranium). Developing and implementing an alternative protocol, in coordination with regulators and stakeholders, that recognizes that traditional risk assessment approaches are unsuited to such contaminants will enable better discrimination between cleanup actions. In many cases, the calculated risk is dominated by the mere presence of one of these elements near its background level. Thus, the large factors of risk reduction achieved for cleanup of actual waste are masked by the high baseline risk associated with high concentrations in the natural environment. 
Furthermore, the criteria for defining when cleanup has been achieved are similarly difficult and can lead to high cost for continued action. Specific needs related to this issue are seen for mercury (especially with regard to emerging low $\mathrm{ng} / \mathrm{L}$ surface water standards), arsenic by ingestion in risk assessment, and the presence of high levels of natural uranium in some settings. More such standards for other elements and compounds are currently being developed - many of these are based new sensitive toxicologic measurements (e.g., endocrine disruptors, DNA analysis, enzyme expression). The issue of synergistic (nonlinear) interaction among contaminants in a mixture is a closely related topic. Known/suspected nonlinearities and interactions should be investigated and incorporated into decision support tools.

There is a need for technically defensible protocols to evaluate clean up in terms of risk change (or excess risk above baseline) and reducing concentrations to levels that are "as low as reasonably achievable" and to account for synergistic interactions at sites with mixtures of contaminants. There are related needs to improve the science underpinning the emerging toxicological and epidemiological methods and evaluating the significance of the observations. Finally, for both natural and anthropogenic elements, understanding the controlling geochemistry and chemical speciation and the impacts of these on toxicology and mobility are needed. Such studies might serve as the basis for cost effective natural analog based stabilization actions.

\section{Links to other Technical Targets}

Acceptable progress toward this target depends on making key advances in fate and transport modeling as described in "Advanced Environmental Modeling" and in developing and implementing performance verification methods that are robust and accepted as described in "Methods to Verify and Validate Performance". Credible progress toward this target ("Improving the Technical Basis for Setting Remediation Goals") is essential to proper implementation of technologies embodied in all of the cleanup targets and to future success of the overall DOE EM program. 


\section{Methods to Verify and Validate Performance}

Team: Eric Lindgren, Caroline Purdy, Ahmet Suer, Tyler Gilmore, James Murphy, Mike Powell, Tom Ivory and Doug Burns

\section{Summary of Need(s):}

Long term monitoring (LTM) of waste units and remediated sites at DOE facilities is a significant challenge and primary projected cost in the out-years at EM, due to regulatory requirements for extended compliance monitoring. This verification / validation target recognizes the need to deploy better short term performance monitoring systems and to develop alternative verification and monitoring technologies and strategies to monitor and ensure the long-term stability of waste sites. Long-term monitoring requires a wholly different approach from the short-term monitoring, which relies on sensors, sample collection and subsequent laboratory analysis. These short-term methods are not appropriate for long-term monitoring. Approaches to long-term monitoring will require new and innovative strategies and technologies to create dependable, durable and cost-effective monitoring networks capable of monitoring waste sites for decades to centuries. Solutions for long term monitoring will require a shift in approach to emphasize large scale volumetric measurements, indicator parameters and measurements that relate directly to risk (e.g., flux versus concentration). Such shifts will reduce costs and number of monitoring points while providing increased confidence to the public. In addition, development of the recommended strategies for long-term monitoring will require linkage to advances in predictive modeling. "Advanced Environmental Modeling" incorporates traditional fate and transport predictions combined with use of natural analogs to verify long term stability of the remedy or containment system. These methods will help select appropriate remedies or containment systems as well as assisting in designing and verifying the associated monitoring. Long-term monitoring will also require the identification of probable failure mechanisms and leading indicators to observe these failures. This may require that remedies and containment systems be designed to include built-in failure indicators to enable cost-effective detection and response.

In general, short term performance monitoring of active remedial action performance employs existing baseline methods of collecting samples from drilled wells followed by laboratory analysis, which is time consuming and costly. Implementing alternative short term verification and monitoring technologies, preferably in-situ methods, field screening methods, and surrogate measurement methods will allow data to be collected at a substantial cost savings. As noted above, the requirements for monitoring technologies used to verify active remedial performance objectives are quite different from the requirements and considerations for monitoring technologies used after site closure over long periods. Improved short term performance monitoring/verification can generally be realized through improved application of existing technologies and systems (or emerging sensors from the characterization research and development activities) and should not require significant new investment. Nonetheless, implementation using improved methods is important because it will supply data to support future decisions to modify 
design/operation and to complete cleanup activities - because of timing, short term performance monitoring is particularly important to the current "closure sites". The target development team felt that technical progress related to improved short term performance monitoring could be provided through SCFA technical support efforts and should be explicitly embodied in integrated deployments such as ASTD. For this reason, the Scientific and Technical Objectives section primarily addresses Long Term Monitoring development issues.

Specific technology development needs are:

- Develop long-term monitoring systems that are durable, cost-effective and low maintenance

- Systems to measure fluxes, volumetric measurements and/or indicator parameters

- Monitoring systems designed to detect false positives (as opposed to the current false negative systems)

- Develop long-term predictive performance indicators based on natural analogs and/or fate and transport models

- Identify failure mechanisms and leading indicators to observe these failures in remedies and containment systems

- Develop built-in failure indicators to enable detection and response.

- Develop systems that provide for the long-term collection, authentication and protection of data

\section{Relevance:}

Approximately 142 of the 339 STCG needs statements addressed within the SCFA are related to verification and validation or characterization. About half of these are for improvements of sensor and detection methods for specific contaminant(s). The sensor needs include approximately 30 for radionuclides, 5 for contaminant metals, and about 20 for organic contaminants. The remainder of the needs are for general detection capabilities, system infrastructure, and sensors/systems for hydrogeologic parameters, microbiology/geochemistry processes, fluxes, and surrogate parameters. This latter grouping may be of particular interest for long term monitoring applications. All of the 86 contaminated ground waterplumes identified in the DOE complex will require verification of cleanup and acceptable risk performance over tens of years beyond final cleanup at a total projected cost of $>\$ 2.077$ billion. This cost does not include closure monitoring of UMTRA facilities, or vadose zone soil at remediated sites, landfills or other engineered containment units. ALL remediated facilities or sites require comprehensive, but cost effective, LTM technologies. Additionally, stakeholders will expect updated assurances of continued closure status.

\section{Status:}

The current LTM baseline method used today across the DOE complex continues to be drilling wells, quarterly sampling and standard laboratory analysis. Field analytical 
methods have been fully developed and field tested under independent performance evaluation programs. But these methods continue to play a minor role in monitoring programs today. A recommendation from the LTM Sensor/Analytical Workshop held in June, 2001 was that there should be greater use of these methods for monitoring programs in the near term, particularly for metal and radionuclide monitoring during active remediation actions.

There has been only marginal development of contaminant environmental sensors. This is largely due to a lack of focused R\&D programs. Neither DOD (SERDP or ESTCP) nor EPA have developed concerted sensor research programs. The DOE CMST program focused primarily on sensors incorporated in the CPT or other Direct Push drilling techniques. One program that has become a model for networked subsurface sensors for contaminated sites is the E-SMART system developed under AFRL, DARPA and DOE funding. The present system being tested at Tinker AFB polls 100 sensor variables in ten-minute intervals (www.ga.com/advanced_technologies/environmental_technologies).

As suggested by the example above, research efforts to date have principally focused on short term performance monitoring. Near term advances are possible based on straightforward integration of samplers, sensors, data acquisition, data authentication, data transmission, data quality checks, and data presentation. The final verified data needs to be accepted and understood by stakeholders. To meet near term needs, existing industry standard data collection and transmission systems can be integrated with automated sampling and sensor detection systems. Several environmental sensors have been, or are currently being, developed (SNL, Georgia Tech, GE, ORNL, etc.) and some have been tested in the field (Burge TCE sensor). Despite a period of significant investment, none of the listed sensor/systems have met all of the requirements of self calibration, MCL detection levels, continuous operation in the subsurface for extended periods of operation, etc. expected for environmental sensor systems - even to meet relatively short term verification needs. Thus, it is critical that future long term monitoring work focus on new-creative-realistic approaches to gather and synthesize information. Methods that will provide a compelling measure of the environmental risk status and that cost effectively supports a high degree of credibility.

\section{Vital Scientific and Technical Objectives:}

\section{$\underline{\text { Summary }}$}

This verification / validation target requires the development of alternative verification and monitoring technologies and strategies to monitor and ensure the long-term stability of waste sites. The objective of this technical target is to develop the techniques and tools required for the closure and monitoring of remediated waste sites and waste containment systems. The targets, therefore, are to develop a predictive capability to determine long term effects of the remedy and/or containment system, identify failure indicators and develop reliable monitoring systems that can detect these indicators. The sections below identify important topics related to both closure and long-term monitoring themes. As noted, the required solutions for long term monitoring require a shift in 
approach to emphasize large scale measurements and measurements that relate to risk (e.g., flux versus concentration) to reduce costs and number of monitoring points while providing confidence to the public.

\section{Use of indicator parameters}

When considering volatile organic constituents in the vadose zone, the soil gas phase is favored and is easy to sample; therefore, it is most practical to focus on soil gas for these compounds. For nonvolatile contaminants (primarily inorganic contaminants), the story is different. In the vadose zone, soluble nonvolatile, primarily inorganic, contaminants reside in the pore water, which is very difficult to sample and monitor. Because of the difficulty encountered in directly sampling nonvolatile constituents in the vadose zone, the monitoring of indicator parameters, such as soil moisture and electrical properties, is of great utility.

\section{Better temporal design}

The determination of the spatial and temporal requirements for monitoring at a specific site requires a detailed understanding of the geohydrologic setting. Conceptual models, contaminant transport modeling, risk assessment and monitoring objectives will likely be required to optimize the monitoring system design.

One way to see the difference between process optimization monitoring during clean up and long-term monitoring is to consider the temporal, spatial, and data quality requirements of each. Process optimization monitoring is conducted during the active remediation phase when contaminant concentration in the soil should be changing, so more frequent sampling is required over a relatively short period of time. Since active remediation or containment construction target a specific region of contaminated soil, the spatial requirements for where to monitor are better defined. Furthermore, since the desired information will be used to control the process, more detailed analyses will be required, such as accuracy and precision of absolute contaminant. Because of the relatively short time period over which process optimization monitoring is conducted, labor intensive, in field measurements are more acceptable. However, some passive remediation approaches such as bioremediation and monitored natural attenuation can proceed over longer time periods creating a need for sensors that provide information about the presence and expression of a particular remediation process (for example a sensor that tracks a biodegradation process).

Long-term monitoring, on the other hand, is expected to continue for years, if not for decades. The data quality requirements for long-term monitoring are less stringent than for process optimization monitoring. With long-term monitoring, the concern is not so much with the precision and accuracy of an individual point measurement, but with the certainty that a relative change has been detected. The idea is to monitor for changes that signal developing or existing contaminant migration. For the most part (with the exception of containment failure), these changes develop slowly so that less frequent sampling is justified, but is required over a much longer period of time. The long time 
WSRC-RP-2002-00077

Revision 0

Page B10 of B61

frame and repetitive nature of sampling for LTM strongly suggests the need for a system of simple sentinal sensors that can detect the pertinent changes.

Coupling point data and multidimensional methods for key contaminant types

The contaminant type bears on spatial sampling requirements. Pore water migration paths in the vadose zone are difficult to predict with certainty, and fingering can result in large fluxes of soluble contaminants in small spaces. Therefore, the spatial requirement for monitoring soluble inorganic contaminants with low volatility in the soil pore water, can only be achieved with a dense data grid over a large area. It is not likely that achieving the required data density using point measurements is practical, so the use of multidimensional methods for obtaining the needed data density, such as geophysical or remote sensing methods are suggested. There is a need for the development of synergistic coupling of point indicator sensor measurements with spatially distributed multidimensional measurements as a way to achieve the spatial and temporal monitoring requirements with a sparser grid of sensors.

The situation is different for volatile organic contaminants. When soil moisture is low, the larger size pores are air-filled, which permits relatively fast vapor phase transport by advection and diffusion. Soil gas migration precedes pore water migration and tends to be more evenly dispersed and predictable in the subsurface. Therefore, the spatial requirement for monitoring organic contaminants in the soil gas can be achieved with a less dense grid. There is a need for the development of simple sensors that can detect the pertinent changes in vapor VOC concentrations. These sensors need not provide detailed chemical information, a total VOC sensor would be adequate.

\section{Access Technologies}

\section{Access Technologies and techniques are a vital need of any long-term monitoring} program,. Placement, replacement and periodic calibration of monitoring tools or sensors require access to the subsurface. Current subsurface access is through wells or boreholes. CPT or other direct push technologies can also be used for direct placement of embedded subsurface sensors Push technologies offer lower cost placement capabilities and can be removed easily if required with the added benefit of not generating drilling waste. Future advancements in monitoring techniques may include alteration of the subsurface through injection of materials or sensors or alternatively, the use of indirect measurement such as geophysical techniques, or remote sensing.

\section{Technology Insertion}

The idea of technology insertion is an important one for reducing the lifecycle cost of long-term monitoring. It is quite reasonable to assume that many technological advancements will be made in sensing and monitoring over time. As these advancements occur, these higher performing, lower cost approaches can be incorporated into new and existing installations. Future retrofitting of existing installations can be facilitated by 
designing with anticipated changes in mind. Conformance to industrial communication standards/protocols is of vital importance.

\section{Monitoring System Design: Cost and Trust}

The key to closing and transitioning a waste site into a long-term monitoring program will rely on the confidence that the regulators and stakeholders have in the remedy or containment system. This confidence will be built on having a predictive capability of the long term effects, identification of failure indicators and a reliable monitoring system that can detect these indicators. In order to develop a cost-effective system, the monitoring of failure indicators must be gathered by passive or simple field screening methods. These systems must be highly automated or rapid and low cost. They must screen for changed conditions in a manner that is easy to understand and present. The information must be made available at a central location for further analysis and archive. A high degree of automation can be achieved through the synergistic combination of sensors for measurements and periodic multidimensional measurements to provide information between the measurement points. The design of a long term monitoring system should also build trust and confidence in the system being monitored. Stakeholders and regulators need fully authenticated and scientifically defendable information that remedial actions are safe for future generations. The gathering, archiving, analyzing and most importantly, openly distributing information, builds trust.

\section{Long-Term Administration of Sites}

In long-term monitoring, there will be an enormous amount of significant information in digital form. These data are vulnerable on many levels. Because of the increasingly fast cycle of obsolescence in hardware and software, we are at the point where the proliferation of electronic data on various platforms has prompted some serious concerns about the long-term protection of the data. A number of international organizations are examining technological issues that bear on the problem, including data types, media stability, and options for refreshing and migrating data to ever-evolving platforms. It will be critical to have a data archival system that addresses these issues.

\section{Links to other Technical Targets}

Acceptable progress towards this target depends on making key advances in fate and transport modeling as described in "Advanced Environmental Modeling" and in developing robust and reliable sensors for key contaminants as described in "Techniques and Technologies that support Characterization". Credible progress toward the target "Methods to Verify and Validate Performance" is essential to proper implementation of technologies embodied in all of the cleanup targets and to support "Improved Technical Basis for Setting Remediation Goals". 
WSRC-RP-2002-00077

Revision 0

Page B12 of B61

\section{Organic Source Zone Treatment or Stabilization}

Team: Tom Early, Tyler Gilmore, Jim Melton, Rich Smalley, Jay Brown, Roger Aines, and Brian Looney

Summary of Need(s):

A primary DOE need is to treat organic contaminants in source zones. This is critical because these contaminants exist at nearly every DOE facility and pose a significant and long term threat to human health and the environment. Further, the most important step in reaching a defensible-sustainable remedy for these sites is to eliminate the source terms. Current technologies for treatment in the source zones are for the most part inadequate because of high costs and uncertain performance. To address this issue, refinement of current technologies and development of strategic new ones will be required. In addition, a better understanding of how the processes that affect contaminant distribution, migration and treatment are fundamentally connected to characterization and access and delivery systems is needed.

This target advocates continuing the past SCFA progress in moving through the National Academy of Sciences' chart of remediation challenges from less difficult to more difficult. Thus, this target recommends (in priority order): continued collaboration with other agencies and programs to advance practical application of existing organic source zone treatment methods and development of entirely new source zone treatment and stabilization technologies for more challenging systems/contaminants. The focus for future activities is on enhanced removal and in-situ destruction technologies for conditions that are more difficult than those already addressed by SCFA (fractured systems and low permeability media). Issues highlighted in this target include development and selection of techniques that are compatible with a transition to less aggressive and more passive polishing of the site - for example, technologies that do not foreclose on biological remediation because they cause long term sterilization of the subsurface. Final concepts described in the target include key linkages to other technical targets. These linkages include the need for characterization tools to designate and delineate the source zone so that expensive and aggressive cleanup methods are not applied in areas where they are not needed. The linkages also include advanced environmental modeling tools that focus specifically on projecting contaminant releases under a range of conditions/treatments, and developing a reasonable technical basis for selecting end states and setting cleanup goals.

\section{Status:}

Organic contaminant sources account for 111 of the 176 plumes or roughly over $60 \%$ of identified plumes at DOE facilities across the country. Of these, 101 Plumes contain are volatile organic compounds (VOC), 5 are plumes of explosives residue, and 5 are hydrocarbon fuel plumes. These statistics establish the essence of the problem. 
Organic source zone treatment has been the subject of intensive R\&D activities at DOE and other federal agencies over the past $10-15$ years. For example, treatment of contaminants associated with fuels has been the subject of many studies conducted by DoD and EPA. Extensive work with VOCs by DOE, EPA, and DoD has focused on technologies applicable to DNAPLs in the vadose zone (SVE) and both the vadose and saturated zones (thermal technologies). In addition, DoD and EPA continue to investigate chemical flushing technologies, while DOE has concentrated on oxidation treatment methods applicable to DNAPLs. Basic science investigations into DNAPL fate and transport behavior in the subsurface have been pursued by DOE (EMSP,OBER), DoD (SERDP), EPA (ORD), and many university- funded programs such as EMSP, HSRC, and NSF. The DoD (US Army) continues to develop methods for addressing explosive contaminants. Additional R\&D in DOE, DoD, and EPA focused on the behavior and treatment of PCB contamination in source regions has occurred, but not at the same level of activity as for DNAPLs.

The current status of these R\&D efforts leads to the following conclusions:

- There are no proven, cost-effective solutions for treating organic source terms with the possible exception of DNAPLs in the permeable vadose zone (SVE) and fuel contaminants in some environments (SVE, biological remediation).

- Heterogeneities present in contaminated geologic media continue to represent a significant barrier to effectively treating many sources.

- Contamination in locations with low permeability, unconsolidated media (i.e. clayrich soils) and complex geologic settings such as fractured rock and karstic limestones are particularly difficult sources to address.

- Source characterization, access, and treatment delivery systems are among the most important ancillary factors where improvements will directly impact the design and implementation of cost-effective treatment technologies.

- With the possible exception of PCBs for which sorption can be an effective stabilization process, little or no progress has been made to explore source stabilization technologies for the other organic contaminants.

The state of the supporting science for this target is weak and requires focused effort from linked targets such as "Advanced Environmental Modeling". Models that work for extreme environments and that respond in a completely scientific manner to the classes of organic source zone remediation (steam, aggressive redox reagents, and the like) do not exist. Such work is a key element in the current SERDP call for proposals. Characterization tools support potential progress in this area. The science is poised to go from theory to application at this time. There has been a significant historical investment in organic source zone remediation that provides an initial set of remediation tools to analyze. There is promise in comparing these options using defensible models that project future releases to resolve the difficult question of the value of partial versus complete source removal. 


\section{Relevance:}

Although recognized as a major contaminant issue for at least the last decade, the current suite of technologies to treat or stabilize the source zones of the organic contamination have been shown to be inadequate to fully address the problem. Current remedies for these source terms have focused on active or energetic remediation schemes such as thermal treatment, solvent flushing, and oxidation techniques. While these techniques hold value, their success in some cases has been limited because of the unique physical and chemical properties of these contaminants. Their movement and distribution in the environment are difficult to determine and their chemical properties, coupled with the physical properties of the contaminated media, makes stabilization, extraction or destruction in place difficult. While solutions to this problem may be through the refinement or the effective application of a suite of technologies in a strategic fashion, there is a need for new technologies such as those that can passively or semi-passively address the organic source terms. Those areas that hold potential for development are diffusion-based technologies and technologies or methods that can more effectively deliver reagents to stabilize or treat the organic contaminants. For example, nontraditional reagents such as, but not limited to, gases and colloidal-sized particles may be effective. These technologies should also utilize or take advantage of natural processes where possible.

\section{Vital Scientific and Technical Objectives:}

Progress in addressing this technical target depends upon pursuing the following complementary approaches, all within the central themes of continued collaboration with other agencies and organizations and developing new technologies for more challenging conditions:

- Evaluate R\&D progress within DoD for explosive contaminants and both DoD and EPA for fuel constituents to determine what gaps exist relevant to related contamination at DOE sites; coordinate future activities

- Continued full-scale field testing of selected, relatively mature DNAPL source treatment technologies (thermal, oxidation, and chemical flushing) to identify gaps in our understanding of engineering design and scientific processes that when addressed will lead to significant improvements in their future implementation

- Identification of entirely new technical approaches to organic source zone stabilization and treatment that involve new applications of existing concepts or technologies evolving from a more refined understanding of physical, chemical, and biological properties of the contaminants as they occur in the subsurface

- Pursuit of technology trains or systems of treatment technologies that will optimize the cost-effectiveness of treatment

- Development of effective process monitoring methods to yield real-time information on treatment progress in heterogeneous systems 
- Integration of the "systems approach" to treatment that recognizes the critical importance of source characterization, containment/treatment/stabilization, and longterm monitoring to successfully address contamination

- Recognition that integration of DOE activities with closely related efforts of federal $\mathrm{R} \& \mathrm{D}$ programs in other agencies associated with this technical target is essential

The success of these approaches depends upon proper staging of R\&D activities. Some activities are near term in nature to address immediate needs for treatment technologies, whereas others are mid- and long-term goals and focus both on making significant improvements in existing or entirely new technologies and on an improved understanding of relevant physical, chemical, and biological processes.

\section{Near Term:}

There is a need to develop the most promising current technologies that end users will use. Therefore, more testing of a select few workable technologies is needed to continue in the near term. Technologies required are improved delivery systems that more effectively deal with heterogeneities; better characterization methodologies for determining the distribution of contamination to target for treatment; more cost-effective process monitoring technologies; and new biological treatment processes for DNAPLs. Emphasis is also needed on developing and testing treatment trains and coupling primary and polishing technologies as components in a system and on developing and testing source stabilization approaches.

\section{Mid Term Strategy:}

Identify break-through treatment and/or stabilization technologies. Develop current laboratory-scale technologies at pilot-field scales. A number of technologies should be tested in the field for proof-of-principal testing. These pilot-scale projects can be relatively low-budget to maximize the testing of promising laboratory-scale technologies such as diffusion-based technologies and systems for injecting "non-traditional" type reagents (gases, colloids, etc.).

\section{Long Term Strategy:}

Develop the science needed for newly identified technologies and address fundamental science questions related to fate and transport in the environment and further understanding of current technologies to support refinement.

Another overarching or unifying consideration applies especially to field testing activities. The $1997 \mathrm{NRC}$ report on remediation technologies identifies the difficulty of treatment of contaminants as a function of contaminant type and media properties. For PCBs, effective treatment is extremely difficult in all types of geologic conditions. For VOC and fuel constituents, relatively permeable and homogeneous vadose zone settings present few treatment problems, and our strategy is to limit future activities under these conditions. Beyond that, however, the most relevant progression of testing conditions 
involves an increasing degree of heterogeneity (both in the vadose and saturated zones), clay-rich media, and bedrock where dual-porosity systems may occur. In general, stabilization and treatment activities will be staged to reflect this progression.

\section{Synopsis of End-User Needs Associated with Target:}

Almost all DOE Sites are impacted, and a large portion (60\%) of the contaminant plumes across the complex have organic contaminant sources. Treatment benefit fact: each gallon of DNAPL that is destroyed reduces pump and treat volume by 300 million gallons. As a result, the chemical destruction or immobilization of contaminants may significantly reduce pump and treat costs and reduce the length of treatment, accelerating the closure of sites.

Access and delivery methods are among the most important factors for designing effective stabilization and remediation processes. Methods that provide the ability to effectively detect subsurface VOCs and map plumes without costly and complex drilling sampling and monitoring wells are needed.

Continued focus on treatment and stabilization techniques, with a consistent effort toward annual updating of findings from the evaluation of pilot demonstrations regarding technology performance, is necessary to continue critically important progress toward identifying new technologies for broad application and overall improved path forward. Evaluation of the end-user needs statements resulted in five summary topics:

1. Access and delivery methods are among the most important factors for designing effective stabilization and remediation processes. Methods that provide the ability to effectively detect subsurface VOCs and map plumes without costly and complex drilling sampling and monitoring wells are needed.

2. Remediation of large plumes contaminated with TCE, PCE, PCBs halogenated organics, and other organic contaminants such as solvents are a major challenge and require non-invasive technologies with high resolution to define anomalies (e.g., DNAPLs on a scale of centimeters or inches). Also rapid cost effective remediation technology that can perform a well-controlled VOC, DNAPL and LNAPL removal from finer-grained sediments and groundwater is needed.

3. Removal of subsurface VOC contaminants in low permeability soils and fractured rock, especially for deep reactive zones for groundwater treatment where bioremediation and surfactants are not practical, requires new cost effective options. Electro-osmosis and thermal technologies are considerations.

4. Non-invasive removal of PCBs from soil above and below water tables underneath buildings without volatizing contaminants in buildings (pump and treat is causing migration) is needed. 
5. An effective method for cleaning up soils contaminated with High Explosives (RDX, TNT, and HMX) is needed.

\section{Links to other Technical Targets:}

Acceptable progress towards this target depends on making key advances in fate and transport modeling as described in "Advanced Environmental Modeling" and in developing efficient "Access and Delivery" methods. This target also relies on developing and implementing improved decisionmaking and performance verification approaches as described in "Methods to Verify and Validate Performance" and "Improving the Technical Basis for Setting Remediation Goals". 
WSRC-RP-2002-00077

Revision 0

Page B18 of B61

\section{Metals and Radionuclides Source Zone Stabilization and Treatment}

Team: Mike Kelley, Jay Brown, Doug Burns, Van Price, Rich Smalley, Roger Aines and Brian Looney

\section{Summary of Need(s):}

The focus of this target is on treatment/removal approaches and techniques that overcome the issues of complex waste near the source and facilitate transition to more traditional (i.e., lower cost) techniques and/or to document that residual is stabilized to protect human health and the environment. This target is quite different than the one related to organic source zones because of the fundamentally different nature of radionuclides and metals. In this case, technology development leading to stabilization is emphasized for metals because, in most cases, transport is slow and exceedances of standards (e.g., groundwater concentrations) are relatively low. Important, but exceptional, cases where geochemistry is highly perturbed (high level waste tanks at Hanford, uranium in groundwater at Oak Ridge) are important and the issue of understanding and manipulating chemical speciation is a vital theme. The target recognizes that physical containment is a viable option for relatively small source zones.

Department of Energy activities have generated large amounts of metal and radionuclide contaminated wastes. Metals and radionuclides associated with these wastes are now migrating into the environment as the result of natural transport processes and are a potential source of risk to human health and the environment. Various research and development projects associated with the Department of Energy, the Environmental Protection Agency, and other public and private organizations have developed technologies that are capable of limiting metal and radionuclide migration. Unfortunately, the long-term effectiveness of most of these technologies has not been studied enough to make them acceptable to regulatory agencies and concerned stakeholder groups. As a result, many of DOE's remediation decisions default to source excavation and retrieval and the use of groundwater pump and treat systems to address regulator and stakeholder concerns. These processes have the following significant shortcomings:

- They can be very costly

- They typically produce secondary waste streams

- They produce risks to workers who have to implement the processes

- They transfer risks from the original release or disposal site to other sites where the wastes are stored or treated

- They may not meet remediation goals, or they may take a very long time to meet these goals.

This target has one primary goal: to increase the body of knowledge associated with alternative source zone stabilization and treatment technologies. This work must focus on two themes: 
WSRC-RP-2002-00077

Revision 0

Page B19 of B61

1. Identifying alternative technologies and the end-point performance criteria that can be achieved by each under varying field conditions.

2. Identifying appropriate methods for verifying that a technology has been implemented properly and that it will remain effective for long periods of time.

Achieving these goals will require a greater understanding of how natural processes affect metal and radionuclide sources. This understanding is needed so that undesirable side effects of stabilization and treatment technologies can be avoided and so that the technologies can take advantage of beneficial natural processes that limit contaminant mobility.

\section{Relevance:}

Applying proven and accepted technologiès (pump-and-treat and excavation) for stabilization and treatment of all defined M\&R source areas across the DOE complex would require unrealistic, unacceptable and unlikely financial resources. Past practices that were considered the 'best' practice have been inflexible in design and implementation in the face of evolving innovative technologies. These past practices have also tried to engineer stabilization and treatment systems that do not always account for key natural processes occurring at the remediation site. Waste minimization and life cycle cost often were not considered when designing and planning stabilization and treatment solutions to M\&R source zones. In addition, these engineered systems often do not consider, or make unrealistic expectations of, the final remedial state of a site. A different strategic approach to $M \& R$ source zone stabilization and treatment is required.

\section{Status:}

Typical metal and radionuclide source zones that can contribute to further contamination of soil, groundwater, vegetation, and surface water within the DOE Complex include: INEEL RWMC buried wastes and trenches; INEEL TAN injection well; OR injection wells; OR Y-12 mercury; Hanford cribs and trenches; Hanford soil associated with tank farms; LANL augered MDAs; and what we consider sleepers - poorly defined problems that include mercury in the SRS burial ground, beryllium and arsenic across the Complex, contamination beneath buildings such as the SRS canyon buildings, and complex mixtures of contaminants that include explosive or pyrophoric materials.

Remedial technologies exist for a majority of known M\&R contamination, at least in the development stage. Barriers to their implementation include: specific modifications to adapt them to site conditions (which requires understanding site geology and geochemistry); methods of access that assure effective application, especially beneath facilities such as tanks or buildings or in the presence of other constructed interferences or in problematic geologic settings; and, finally, methods to validate their effectiveness.

Many technologies, currently in the development or demonstration stages, are discussed in detail in recent references such as the DOE book Vadose Zone Science and Technology Solutions. Programs that have been active in developing basic science associated with 
metal and radionuclide chemistry and the interactions of metals and radionuclides with plants and animals, or in the application of this chemistry and biology toward stabilization, include EMSP, NABIR, SERDP and ESTCP. Linking to these programs to avoid duplication and transitioning promising basic science into applications should be a focus of future efforts.

\section{Vital Science and Technology Objectives:}

This specific target will advance implementation and acceptance of M\&R source zone stabilization/treatment technologies and provide the criteria for comparing technologies to meet goals. There are three key objectives to achieve this target:

1. Integrate and deploy cleanup systems for metals and radionuclides source zones to:

a. Optimize individual existing technologies

b. Determine the best implementation of multiple technologies in concurrent application or in series for mixtures of contaminants

c. Set criteria for the transition to the next stages of cleanup (especially from active remediation to MNA)

d. Evaluate the potential for, and responses to, unexpected mobilization.

2. Develop and validate metals and radionuclides treatment mechanisms and post treatment stability, including temporal changes during treatments, measurement of treatment efficiency/completeness, and natural analogs. This objective is considered to be a high priority.

3. Develop entirely new source zone stabilization and treatment technologies, considering basic processes for metals and radionuclides under varying scenarios. This is desirable for some contaminants and could be enabling for technetium and others.

The underlying assumptions for this strategy are:

- Source term stabilization/treatment is cost effective and can eliminate future groundwater contamination, source release and risk.

- Viable technologies exist for the majority of environments and contaminants of concern and/or technologies can be developed through application of sound scientific principles.

- Establishment of end states will allow for comparison of technologies with respect to cost, schedule, land use, and goals.

The focus of any practical R\&D effort should be on developing methods that are compatible with a viable-acceptable-verifiable end state. Scientifically achievable end states for stabilization and treatment trains must be quantifiable and acceptable to scientific/regulatory/stake holder communities. Criteria for the longevity waste form must also be established and verified using a combination of natural analogs, laboratory 
and field testing, and empirical techniques. The verification/validation theme feeds into the overarching verification target.

Many DOE sites have needs associated with this target, and the proposed themes would address most of the listed needs at Hanford, Y-12, INEEL, LANL, Pantex, Mound, and SRS.

\section{Links to other Technical Targets:}

Acceptable progress towards this target depends on making key advances in fate and transport modeling as described in "Advanced Environmental Modeling" and in developing efficient "Access and Delivery" methods. This target also relies on developing and implementing improved decisionmaking and performance verification approaches as described in "Methods to Verify and Validate Performance" and "Improving the Technical Basis for Setting Remediation Goals". 


\section{Advanced Sustainable Containment Systems}

Team: Jody Waugh, Tim O’Rourke, Terry Sullivan, Terry Hazen, Bob Aylward and Mike Serrato

\section{Summary of Need(s):}

This target advocates that DOE devise and develop containment systems based on a systems engineering approach. The approach emphasizes the use of natural processes and links civil engineering and natural science methodology (ecosystem engineering) to design, construct, and verify sustainable containment systems Properly applied and monitored, physical containment and barriers will remain a central activity in DOE environmental management at all sites for the foreseeable future. Advancing the science and technology base relatively rapidly is particularly important to closure sites that need to implement and document such systems in the next several years.

DOE plans to use engineered containment systems to isolate long-lived contaminants left or buried in the subsurface during remediation of its nuclear facilities. DOE agreements with regulators and stakeholders require engineered systems that will control migration of subsurface contaminants for 100 s to 1000 s of years, and do so while natural processes are acting to mobilize contaminants. This is an unprecedented engineering challenge. To meet the needs of this area, work is required to develop a system engineering approach to design and verify containment systems. This topic covers a wide range of specific issues including robust design concepts that incorporate multiple lines of defense, natural analogs to help predict performance, material durability, construction practices, and improved risk-based performance modeling.

Conventional designs are collections of prescribed physical barriers to known or perceived release pathways that are rarely evaluated as integrated systems. End users are in need of innovative guidelines for the design, verification and performance assessment of engineered systems that focus, not on barriers to natural processes that will degrade in the long-term, but on enhancing beneficial natural processes that may even improve containment in the long term. New methods are needed to consider effects of inevitable long-term changes in engineered material properties, geomorphology, soil hydrology, ecology, and climate that may impact performance.

Current containment system designs and materials have not proven to be effective for the time frames required to contain the long-lived contaminants needing management by DOE. For example, clay layers within caps and bentonite slurries in walls are known to crack and desiccate under routine wetting and drying conditions in the field. Additional research and development on longer-lived, more durable containment system materials is needed. Current examples of alternative materials include asphaltic concrete and geosynthetic composite layers. Furthermore, new methods and techniques for accelerated testing and field testing are necessary to support long term performance evaluation of containment system materials. 
Scaling issues from taking laboratory and pilot scale tested designs and applying these designs to larger scale containment systems can create problems that impact system integrity. New methods and techniques for quality control and verification are needed to ensure that these systems conform to design specification during construction. In addition, verification testing will provide baseline data for following the evolution of performance through long-term monitoring.

For the containment system, monitoring data will be needed to demonstrate that the system is operating within its design envelope and to support data needs for predictive modeling. The long time frame over which verification data will be collected favors techniques that are durable, easily replaced, non-invasive, provide an early warning that contaminant release may occur, or are representative of large regions of the containment system (areal measures versus point measures). For surface features, remote sensing techñiques are also desirāble. End users have identified verification of containment system performance as an important issue for long term isolation.

Predictive capabilities (computer models or analogues) will be required to support containment system design, risk-based performance modeling, provide input to future decisions on maintenance when the system is not operating as desired, and, supported by monitoring data, to assist in demonstration of long-term isolation. This requires understanding the containment material properties that impact flow and transport and how these material properties change over time. It also requires understanding of the impacts of changes in the environmental setting (climatic changes, extreme weather events, etc.) on containment system performance. Currently, the long-term changes in containment system performance have large uncertainties. These uncertainties lead to costly designs, extensive monitoring requirements, and limited confidence in long-term predictions. This has led a number of end users to identify a need for improved predictive modeling of containment system performance

\section{Relevance:}

Virtually every DOE site will require a long-term containment system for waste left in place or disposed of. Many of these containment systems will require long-term monitoring and performance verification. Current systems are unproven and costly. Improved designs, materials, and performance monitoring will enhance protectiveness and lower long term stewardship costs.

\section{Status:}

Limited field evaluations show that many existing containment and cover systems are failing to meet design standards in the short term. In particular, biointrusion, desiccation, frost penetration, and other soil development processes have increased permeability of compacted soil layers and other resistive materials intended to last for hundreds of years. DOE and other agencies are currently funding short-term field tests of alternative cover designs, primarily for arid and semiarid regions, that rely on a soil "sponge" layer to store precipitation and plants to seasonally return it to the atmosphere. Sandia's Alternative 
Landfill Cover Design (ALCD) project, PNNL's Hanford barrier prototype, and EPA's Alternative Cover Assessment Program (ACAP) studies are examples.

The common design approach for containment systems attempts to extrapolate long-term performance with numerical models calibrated using short-term field data. The UMTRA stewardship project and others are finding that the performance of engineered covers will change in ways that cannot be captured using short-term field data and numerical models. In 2001, DOE's SCFA initiated work that will ultimately yield a cover design guidance that will incorporate reasonable ranges of environmental change and result in more reliable and acceptable long-term containment systems.

\section{Vital Scientific and Technical Objectives:}

\section{Holistic Sȳstems àd Engineering Approach:}

Develop a systems engineering approach to design, construct, and verify containment systems. The approach will emphasize an ecosystem engineering framework that links civil and geotechnical methods with natural science concepts and methodologies for developing a robust containment system. Engineered systems that mimic natural systems known to be stable over long periods of time will be emphasized. The approach contains the following five objectives that support this penultimate objective.

- Evaluate evidence from natural analogs that can be used to 1) help devise sustainable containment systems that imitate natural systems, and 2) project reasonable ranges of environmental change (e.g., climate, geomorphology, soils, ecology) for input to long-term performance evaluations. This is a medium priority.

- Identify and evaluate failure mechanisms impacting containment system performance such as material degradation, geomorphological processes, pedogenesis, and ecological succession. This involves an improved scientific understanding of the impacts of interrelated ecosystem processes on the long-term performance of engineered covers, as well as research and development on durable alternative materials. Alternate material, such as asphaltic concrete, must have durability lifetimes that exceed the current 10-50 year life expectancy and be able to be demonstrated to last up to 1000 years. Accelerated testing techniques to understand long-term evolution and durability of materials is needed. New testing techniques must be able to more accurately simulate the effects of ecological and climatic variation on designs and materials over large time scales to support long term performance assessment of containment systems. Identification of failure mechanisms is a high priority.

- Develop more robust models that a) integrate containment system components and processes and $b$ ) couple performance and risk assessment The models are needed to develop performance standards based on risk assessment and trade-offs between costs of constructing redundant systems initially and long-term maintenance. Researchers should be able to demonstrate that the enhanced capabilities will help build confidence in long-term model predictions and help provide information for riskbased decisions on system design, maintenance and monitoring needs. This is a low priority. 
- Develop a methodology for projecting long-term performance of containment systems that links field tests (e.g., lysimetry), predictive models, and natural analogs. Modeling will form one of the technical underpinnings of performance verification. Researchers should also develop model refinements based on data collected to support estimates of long-term durability of containment materials. Potential areas where uncertainty could be reduced through model enhancement include the role of evapotranspiration in water balance modeling, changes in material performance over time and how that impacts water flow and contaminant transport, the impacts of extreme events (precipitation, erosion, seismic, etc.) on long-term performance, and the role of natural analogs on determining the evolution of natural materials and ecosystems over time. This is a medium priority.

- Develop and deploy verification framework and tools to verify containment system construction and to target leading indicators of performance. The framework will rely on risk based performance measures and will address identification of indicator parameters that supply early warning of potential changes in system performance and the types, frequency, and need for collecting data. Gaining concurrence on the approach from interested stakeholders is key to successfully addressing this objective. This objective is linked to the verification and validation target and is a high priority topic that needs to be addressed to enhance DOE's ability to verify containment in a cost-effective manner. This is a high priority.

New techniques are needed to ensure that containment systems meet design specification during installation. Examples of new construction verification techniques to consider may be laser based quality control tools or fiber optic networks embedded during construction to measure design integrity. Research and development of enhanced delivery systems for installation of deep barriers will enhance containment system integrity

New tools need to be implemented, enhanced, or developed to strengthen long-term verification of containment system performance. Potential areas for development (work coordinated primarily by the characterization target) include:

- $\quad$ reliable, long life (>25 year) sensors (moisture content and other environmental parameters),

- $\quad$ sensors or approaches to directly measure moisture flux,

- remote sensing techniques to determine water balance, evapotranspiration, containment cell geometry, slope stability and subsidence,

- $\quad$ engineered tracers for early detection of performance degradation (for example, desiccation) or addition of tracers to containment systems as part of the design as an indicator of performance,

- natural analog tracers, and

- non-invasive geophysical techniques for leak detection and containment system geometry verification. 
Conclusion

Effective long term containment will require a systems approach. The previous information outlines each component in the system. Of the above topics, the ones with the greatest potential for reduction of risks and costs are the identification and evaluation of failure mechanisms, including information from natural analog studies and material durability studies, and development of a framework for verification of sustained performance.

\section{Links to other Technical Targets:}

Acceptable progress towards this target depends on making key advances in fate and transport modeling as described in "Advanced Environmental Modeling" and in developing and implementing improved decisionmaking and performance verification approaches as described in "Methods to Verify and Validate Performance" and "Improving the Technical Basis for Setting Remediation Goals". Of these, focused containment-related work is needed in the specific areas of construction/performance verification and long term monitoring. 


\section{Integrated Containment-Treatment Concepts - "Smart Containment"}

Team: Brian Looney, Terry Hazen, Tyler Gilmore, Jody Waugh

\section{Summary of Need (s):}

A new "smart containment" approach that incorporates modifications so that solid hazardous and/or radioactive waste is stabilized or detoxified during a period of containment or isolation is needed. To support such a strategic development, potential treatment processes (delivery systems and their compatibility with the isolation systems) must be evaluated, as well as methods to monitor treatment progress and document when the waste containment is no longer needed.

\section{Relevance:}

The relevance, and potential benefits, from addressing this target are exemplified by recent technical assistance activities throughout the DOE complex. In complex situations where technical assistance has been requested, the SCFA Lead Lab has routinely identified a smart storage option that provided a high level of performance at a significantly reduced cost. Specific examples include the Lead Lab Technical assistance reports to Sandia National Laboratory (PCB contaminated soil), to Lawrence Livermore National Laboratory (site 300), to Brookhaven National Laboratory (viscous barrier), and others. The significant potential value for smart contianment has also been highlighted in other technical reports such as the technical evaluations of detrititation and tritium management strategies (both at Savannah River Site and Hanford). These highlight the potential for eliminating tritium risks by recycling highly contaminated water for use in cements and grouts for tank closure. The potential relevance for smart containment is supported by the fact that there are over 3500 municipal and solid waste landfills in the United States, with over 100 lined and unlined landfills in DOE. These are currently slated to receive large volumes of solid waste generated by environmental restoration activities.

This concept is valuable in part because it eliminates inefficiencies associated with traditional administrative boundaries (e.g., ER, WM, D\&D) and encourages closure of the WM-ER-WM cycle. The primary benefit of this target strategy is elimination of the need for maintaining and documenting the performance of containment and isolation systems for 100 s to 1000 s of years. Integrated smart containment provides a highly desirable option that meets end-user needs in both the short and long term.

\section{Status:}

Traditional response actions for solid radioactive and hazardous waste in the environment fall into four categories: containment by emplacement of engineered barriers, in-situ stabilization or destruction, removal followed by ex-situ treatment, and removal followed by storage and disposal. Ex-situ treatment generally consists of separation methods for volume reduction and/or engineered stabilization/detoxification facilities (normally 
"expensive" thermal, chemical or biological treatment systems). An important strategic target is development of a new option, a modified "smart containment" concept. Such systems are designed to make technically based modifications to the storage/disposal environment to integrate low cost "passive" natural treatment. The value of this concept is that the treatment process built into the smart containment system would eliminate the need for "permanent" monitoring and maintenance. Indeed, this concept represents a critical step to improve long-term stewardship by eliminating future hazards. The potential benefit of this general class of action is indicated by several historical efforts:

- Success of biopiles as an example where the treatment occurs rapidly and completely versus attempts to treat in situ or by shipment to landfills (many EPA reports)

- Information on emerging municipal landfill research/concepts (see, for example, LBL reports related to T2ALF model)

- A growing body of potentially applicable scientific literature on compounds that will degrade slowly under appropriate conditions (e.g., PAHs, PCBs, pesticides, and the like)

- Many past examples - efforts to isolate short-lived radionuclides to allow time for decay

This target moves beyond the artificial and regulatory dichotomy currently in place that allows either "permanent" containment or expensive hazardous waste treatment facilities. The "dry tomb" concept implicit in most containment and landfill storage forecloses the technical opportunity for an optimal integrated solution because these typically require the presence of water, reagents, or gases and possibly delivery or recirculation activities.

\section{Vital Scientific and Technical Objectives:}

\section{Demonstrate "smart containment" options for solid hazardous and radioactive wastes associated with environmental restoration activities. Develop protocols for} candidate "end-user" waste streams in DOE (as defined by STCG needs statements and other resources). The protocol should identify and consider waste types that are not suited to the concept, such as long-lived radionuclides. The protocols should also recognize and develop a technical basis to overcome regulatory concerns and other challenges. Potentially useful treatment technologies have the following characteristics:

- they have a clear scientific-theoretical basis,

- they exhibit low energy and/or resource use,

- they are compatible with the isolation system,

- they are effective within the projected life of the isolation system, and

- their progress is measurable so that performance can be documented in a cost effective manner.

Importantly, the "smart containment" configuration is not limited to in ground (landfill style) implementation but could also be applied to above ground storage buildings.

Above ground storage is often designed to simply house waste containers for extended periods while final disposition is negotiated. Smart containment would provide an option 
for waste treatment to be underway while the remainder of the decisionmaking process was underway.

Additional work to provide data to facilitate crossover of existing treatment processes, monitoring tools such as sensors, and other required technical elements to support this new concept are required. Development of technically defensible protocols, configurations and monitoring approaches for "smart containment" will facilitate deployment and use of this new and promising strategy.

\section{Links to other Technical Targets:}

Acceptable progress towards this target depends primarily on making key advances in developing and implementing improved decisionmaking and performance verification approachès as described in "Methods to Verify and Validate Performance" and "Improving the Technical Basis for Setting Remediation Goals". 
WSRC-RP-2002-00077

Revision 0

Page B30 of B61

\section{Effective and Sustainable Technological Solutions for Contaminant Plumes}

Team: Terry Sullivan, Terry Hazen, Tom Early, Gerry $\operatorname{Voos}_{2}$ Norine Noonan, Jody

Waugh, Jack Corey, Tony Palumbo, Diane Wilburn and Jerry Brown

\section{Summary of Need(s):}

A central activity in environmental stewardship is effectively managing and cleaning up plumes of contamination that have been released from source zones. This target identifies two themes in which science and technology can assist in both the primary plume zone and in the dilute plume. The target also emphasizes active responses transitioning to less active sustainable actions over time. Several vital scientific and technical objectives remain:

- optimizing active treatment systems (primary plume),

- understanding the relationship between performance and the completeness of source zone treatment (primary plume),

- developing technologies that transition more quickly to monitored natural attenuation through emplacement of long term reagent materials and through identification of measurable natural processes that destroy, stabilize, or detoxify dilute plume contaminants (both primary plume and dilute plume), and

- developing design approaches and viable monitoring strategies (both primary plume and dilute plume).

This topic is viewed as critical to future DOE environmental stewardship performance.

The field of primary plume treatment is relatively mature, and extensive field experience is available. However, this does not mean that improvement of current systems is not warranted. There are also several examples where performance did not meet expectations, including optimizing treatment systems (including optimization of well placement and pumping rate), understanding changes in performance over time for permeable reactive barriers, improving treatment systems for actinides (notably uranium), and understanding subsurface flow and transport (to improve control over redox conditions to enhance bioremediation and to enhance delivery systems for chemical and biological treatment).

There are a large number of off-the-shelf treatment technologies that could be improved. However, the possibility for major improvements is limited. For this reason, three major objectives were identified that have a potential for substantial improvements to primary plume treatment:

- Develop a systematic approach that couples treatment strategies among the source, primary, and distal regions of the plume. In the past, DOE has sponsored several demonstrations that address one part of the plume. This ignores the holistic nature of the problem.

- Evaluate the long-term efficiency of primary plume treatment strategies. DOE has recently begun to use technologies for in-situ treatment of contaminants. These treatment technologies include permeable reactive barriers and in-situ 
biological and chemical treatment. A robust technical and scientific basis for understanding the performance of these treatment systems over time and after active treatment has stopped does not exist.

- Demonstrate in-situ treatment strategies for plumes containing multiple contaminants (radionuclides and hazardous). Approximately $45 \%$ of the DOE identified plumes contain mixtures of radioactive and hazardous materials. Several of these plumes will require in-situ treatment.

There is a growing need for long-term, sustainable solutions to the significant problems confronting the DOE complex (and other sites) for relatively dilute plumes of contaminants that have migrated from the source zone and primary plume. There are several discrete technical needs (these are in relative priority order of importance as investment targets):

- To understand baseline biological, chemical and physical parameters influencing sustainable remediation

- To understand design requirements for sustainable systems

- To understand hydrologic response to various remedies

- To validate performance of technological remedies

- To develop or improve low-energy, low-input technologies to accelerate remediation

\section{Relevance:}

In a recent survey conducted by EM-22, 176 contaminant plumes in a wide range of geologic settings were identified across the DOE complex (excluding vadose zone VOC plumes). Of these, approximately half (93) consisted of one primary contaminant; the remainder contained two or more contaminants. VOCs are found in $\sim 60 \%$, radionuclides in $\sim 42 \%$, and metals in $\sim 20 \%$ of the plumes. Contaminants such as nitrate, explosives, and fuel constituents are found with a much lower frequency in these plumes. Although preferred remedies have not been determined for all of the plumes, the estimated total cost for applying a pump-and-treat option for many of these plumes exceeds $\$ 2 \mathrm{~B}$.

There are potentially large costs associated with decades (or centuries) of remediation. Thus, new knowledge and new methods of addressing this problem have the potential to lower costs and also have the potential to increase the confidence of both regulators and public stakeholders that these contaminants are being managed in a way that reduces risk. In addition, sustainable technologies will be most useful in dealing with these plumes at closed sites (e.g., Rocky Flats) and for the large dilute plumes for which these technologies might be the only cost-effective option for long-term stewardship.

\section{Status:}

A large number of agencies have been funding research in the area of primary plume treatment for many years. Indeed, the state of our understanding of science in this area may be ten or more years ahead of practical field applications (see reports from the National Research Council, EPA, DOE, and others). Agencies currently funding research 
include: 1) DOE Office of Science (Office of Biological and Environmental Research: NABIR); 2) DOE OST (EMSP, SCFA, ITRD, ASTD); 3) DOE Other Offices (Laboratory Directed Research and Development); 4) DOD (SERDP, ESTCP, AFCEE, USACE); 5) EPA (SITE, RTDF, Kerr); and 6) Other agencies (USDA; USGS). As an example, good resources covering the state-of-the-science and state-of-the-practice for phytoremediation technologies are posted at the RTDF web site for the Phytoremediation of Organics Action Team (http://www.rtdf.org) and SCFA Proceedings from the Workshop on Phytoremediation of Inorganics. Both are products of interagency collaboration with academia and industry. Given the large number of agencies that are conducting research in this area, there is a great need to improve interagency coordination. More coordination of all activities in this area across DOE offices and programs and across other agencies would improve leveraging of SCFA efforts and avoid redundancy. SCFA should focus its efforts on practical applications that are specific to DOE $\bar{E} M$ needs for making remedial strategies more effective, reducing negative impacts of treatment strategies, and enabling monitored natural attenuation and long-term stewardship of DOE contaminants.

\section{Vital Scientific and Technical Objectives:}

The vital objectives are presented for the primary plume and then for the dilute plume.

\section{Primary Plume}

Demonstrate a systems approach to demonstrate complete solutions to groundwater contamination problems. Coupling source and dilute plume treatment strategies with the primary plume strategy for a complete solution.

Primary plume strategies involve both temporal and spatial components and must be closely coupled to source-term treatment or stabilization. Primary plume treatment measures should be designed as a temporary measure to control further downgradient releases of contaminants within the most concentrated part of the plume for a time sufficient for significant impact of source treatment to have reached the region of the primary plume control system. Likewise, the effectiveness and period of operation of the primary plume treatment system must be taken into account for design and implementation of the dilute plume treatment.

Part of the reason that this systems approach to source/plume treatment is essential is to ensure that treatment measures adopted for one part of the system will not have a negative impact on the effectiveness of treatment in any downgradient part of the system. This approach will require a more integrated planning, modeling and design activity than is currently used at any site. Optimizing the individual treatment components both temporally and spatially will be necessary. An example of a demonstration project that could be performed by SCFA is a DNAPL plume with aggressive source term remediation coupled with active treatment of the primary plume and a strategy for . passive remediation of the more dilute section of the plume. 
Evaluate long-term efficacy of primary plume treatment strategies. After active treatment actions are stopped, the contaminants may rebound or undesirable end states, unrelated to the contaminant, may be created (e.g., VOC rebound, redox shifts, biological changes).

Source removal strategies can have a major impact on strategies for primary or dilute plume treatment. Thermal processes can kill indigenous microorganisms adapted to the contaminants and thereby make bioremediation or natural attenuation ineffective. Oxidative processes can also kill indigenous microorganisms and alter ambient redox conditions both in the source area and in the primary plume such that previously sorbed contaminants (both organics and metals) are now mobilized and creating new contaminants of concern. SCFA should focus research studies on determining the critical parameters that will reduce the impact that source treatment strategies have on primary and dilute impact studies. Efforts in this area could include, but are not limited to, post treatment studies on changes and the persistence of those changes on ambient redox potential, oxygen, hydrogen, and $\mathrm{pH}$ in the treatment area and downgradient primary and dilute plume. Other studies could look at the impact and recovery rate of microbial communities in the treatment area and downgradient plumes, especially those groups of microorganisms that might be important in critical biogeochemical processes (e.g., iron reduction) and those capable of degrading the contaminants of concern (e.g., TCE oxidizers and reducers).

Another concern in this area is the effect that primary plume treatment strategies may be having on our long-term ability to remediate the plume and on the strategies possible for the downgradient dilute plume. For example, biostimulation of TCE reduction via reduction (e.g., lactate injection) in a oxidative environment can elute previous sorbed organics and metals in the treatment area. Another example is the long-term stability of reductive treatment zones in naturally oxidative environments. The potential for remobilization of reduced contaminants as the environment returns to its natural state needs to be evaluated in terms of dilute plume treatment strategies. Hydrological control and pump-and-treat strategies (especially those involving reinjection of treated water) may result in well fouling (mineral and biological) or alteration of the normal redox conditions of the environment. These effects can limit the strategies possible for the down gradient dilute plume but also create new problems and greatly increase the time required to treat the primary plume and its cost. SCFA should focus research studies on the following areas to help determine those critical parameters that might alter the efficacy of primary plume treatment (e.g., injection well fouling, redox alterations of the environment, causes for rebound of VOCs after primary plume treatment):

- Determine the critical parameters in source treatment that will alter the environment for treatment strategies in the primary plume and in the dilute plume (e.g., biological recovery, ambient redox potential alteration).

- Determine the critical parameters in primary plume treatment that effect the longterm efficacy (e.g., injection well fouling, VOC rebound, redox alteration).

- Demonstrate in-situ treatment technologies for plumes containing co-contaminants, e.g., radioactive and hazardous contaminants (VOCs). Due to the differences in physical and chemical properties of the contaminants, a treatment technology for one contaminant may not be successful for another. In some cases, the treatment process 
may mobilize other contaminants. Current in-situ treatment technologies are often limited by problems associated with delivery, long-term durability and an incomplete understanding of the impacts of treatment on future conditions. Technologies selected for demonstration should show how all contaminants will be treated, address technical issues that limit current technologies and show an understanding of the impact of treatment on the distal plume. For example, creating a reductive environment for microbial destruction of TCE simultaneous with reduction of metals to an immobile form could be one approach to treating co-contaminants in-situ.

\section{Dilute Plume}

The scientific and technical needs listed above can be addressed through several specific objectives. Leveraging DOE investments through collaboration with other Federal agencies as well as private industry can facilitate all of these objectives.

Enhance baseline understanding: In sustainable remediation systems, organic compounds are usually broken down by microorganisms, metals are usually attenuated by sequestration or transformation by the soil/geologic matrix, and radionuclides are attenuated through natural decay. Research is needed on the relationship between, and the combined effects of contaminant class, concentration, desired end state, and the presence or absence of co-contaminants. Specific information to support successful implementation of innovative technologies in dilute plumes is listed below. Note that this effort is also supported by the overarching targets (setting goals and verification), and by the enabling targets (notably, fundamental processes and biogeochemistry):

- Biogeochmical reactions in the dilute portion of a plume may be more critical to success than reactions in the primary plume. Research is needed on biogeochemical processes throughout a plume (from primary to dilute) for single and mixed contaminants.

- Predictions of behavior (e.g., efficiency of system, meso/macro-scaling at target remedial areas, time of attenuation, transport rate, fate of contaminant) must be improved before stakeholders and regulators will feel comfortable relying on these systems as remediation tools. This is particularly true when a site moves to long-term stewardship based on reliance on natural attenuation or low-input remediation systems.

- Biochemistry of the rhizosphere: research on plant/microbe/soil/contaminant interactions is needed to understand contaminant mobilization and contaminant transformation (enzymatic processes, chelating, etc.) from one compartment or pool to another. Research on the effects of rhizosphere manipulation (inoculation) on the fate and transport of contaminants is also needed.

- Remobilization of contaminants: phytoremediation has frequently been proposed for remediation of buried metals and radionuclides. Before implementation of this potentially sustainable technology, methods must be developed that can quantify more rigorously the risks involved when contaminants are subsequently remobilized into the biosphere from leaf fall, root biomass, herbivory, harvesting debris, etc. Methods are needed to improve the decision process for selection of 
WSRC-RP-2002-00077

Revision 0

Page B35 of B61

phytoremediation by quantifying costs of the long-term commitment needed for this technology.

Document sustainability:

- Develop guidance and protocols for designing sustainable phytoremediation.

Research on plant species selection for phytoremediation has focused on rates and processes of contaminant accumulation. Sustainability requires a new emphasis on remediation ecology that combines information about plant species and contaminant uptake with additional information such as transpiration rates and species tolerance for local climate, soil conditions (e.g., salinity), and intraspecies interactions (e.g., allelopathy).

- Evaluate sustainability of reactive barriers and bioremediation: The long-term sustainability and performance of these systems is unknown and should be an area of technical emphasis. Design parameters for long-term systems must be developed and tested in a variety of matrices.

\section{Validate performance:}

- Evaluate advanced MNA performance indicators for deployment: There are numerous examples (see below) of advanced or emerging remote and in-situ technologies that can be used to evaluate the performance of MNA systems. However, most of these technologies have, themselves, been validated only at a relatively small scale (e.g., laboratory, small field tests). There should be increased emphasis on evaluation of the technical readiness of these methods for deployment.

- Examples of remote sensing methods include multi-spectral reflectance and thermal infrared instrumentation for detecting plant stress, Laser-induced fluorescence spectroscopy to distinguish contaminant-induced and natural stresses. Laser-induced breakdown spectroscopy and x-ray fluorescence of contaminants in plants, genetically-engineered visible markers to quantify contaminants and plant stresses,

- Examples of in-situ technologies: genetic engineering of plants with green fluorescence proteins that signal the presence of heavy metals, phosopholipid fatty acids and other techniques for detecting soil microbial structure and its changes, DNA microarrays to detect stress-related genes in soil microbes

- Verify performance of existing natural attenuation sites. The ability to validate remediation processes is paramount when long-term systems are under consideration. Sites must be able to verify that bioremediation of organic compounds is occurring, and that these compounds are not simply being volatilized, diluted, or transported. Verifying the attenuation of metals and/or radionuclides will require the ability to prove that the contaminants are fixed to the soil-geologic matrix or have been transformed to a non-toxic form. Research is required to develop/substantiate marker compounds (e.g., pristine and phytane for PAHs) or other indicators to validate a sustainable remediation system.

Develop methods for predicting hydrologic responses to alternative remedies: Watershed-scale predictive and monitoring tools are needed to understand the hydrologic response of plumes to remedial actions. Over the long-term, such tools will also be needed to understand changes in the surface environment (climate, ecology, 
geomorphology, soil development, etc) and their impacts on plumes. Further, better predictive and monitoring tools may lead to remedies that include the use of plants to manipulate rates and direction of plume dispersion

Understand and develop new ideas for low-input sustainable systems: Unlike fully active systems used to treat a source zone plume and yet not fully passive, these systems require periodic inputs (e.g., hydrogen peroxide, nutrients, carbon sources) for continued operation. Research should be directed to improving conceptual models for such systems as well as laboratory and field-scale studies designed to evaluate long-term performance.

\section{Links to other Technical Targets:}

Acceptable progress towards this target depends on making key advances in fate and transport modeling as described in "Advanced Environmental Modeling" and in developing and implementing improved decisionmaking and performance verification approaches as described in "Methods to Verify and Validate Performance" and "Improving the Technical Basis for Setting Remediation Goals". This target also depends on success in eliminating both the organic and metal/radionuclide source zones that feed the contaminant plume. 


\section{Tritium Management and Risk Reduction}

Team: Brian Looney, Jim Helt, Ahmet Suer

\section{Summary of Needs:}

The facts that tritium is generated by a wide range of nuclear processes, that it moves freely with natural water, and that tritium separation is challenging has led to the following three general types of end-user needs:

1. "cost effective" tritium treatment (i.e., isotope separation) technologies

2. alternate tritium remediation strategies to reduce releases, exposure and risk

3. tritium characterization and monitoring tools, with particular emphasis on identifying plume arrival or tritium fluxes and risk

This target is organized into two overall themes: the first theme, technologies and strategies to reduce the impacts of tritium releases from DOE sites, comprises the various remediation needs, and the second theme, evaluate and implement methods for tritium sensors, field screening and down-well tritium measurement systems, addresses the monitoring needs.

Note that these themes go beyond the traditional paradigm of considering primarily tritium treatment (hydrogen isotope separation). There is a significant amount of historical research and development throughout the world to support strategic decisions on future work related to these tritium needs. The historical development efforts related to tritium treatment define what constitutes reasonable progress on isotope separation and helps define "cost effective." Significant near-term progress appears feasible related to new management strategies and monitoring.

\section{Relevance:}

One particular radionuclide, tritium, is responsible for most of the population radiation doses from DOE and commercial nuclear facilities. While maximum exposures are generally well below applicable guidelines, DOE and its neighbors and stakeholders recognize the relative predominance of this contaminant and share the goal of reducing and managing exposures to the lowest reasonably achievable levels. The presence of tritium at both large and small facilities, together with tritium-specific properties, result in significant technical challenges and end-user needs. A notable feature of tritium contamination in groundwater and surface water is that the contaminant is present as a part of water molecules. In these systems, tritium, an isotope of hydrogen, replaces one or both of the normal hydrogen atoms in a small fraction of the water molecules. The resulting "contaminated" molecules have properties that are almost, but not precisely, identical to normal water. Tritium treatment, risk reduction and management are identified in approximately 13 end-user needs (Savannah River Site, Hanford, Argonne, and others) and related tritium monitoring is identified in approximately 5 end-user needs. Because of the unique nature/challenges associated with tritium, progress in this strategic target area represents significant potential cost savings. 
WSRC-RP-2002-00077

Revision 0

Page B38 of B61

\section{Status:}

Tritium is present in various wastes and wastewaters at DOE sites and in tritium containing groundwater plumes that range from a few acres to hundreds of acres and at a wide depth range ( 0 to $>1000$ feet). Today, at various DOE facilities, tritium management has been initiated using evaporation, evapotranspiration, or direct discharge.

DOE has periodically evaluated options to reduce and manage the impacts associated with aqueous releases of tritium into the environment (for example, the Savannah River Site tritium treatment report and periodic Hanford technology evaluations to support the triparty agreement). These reports traditionally examine the applicability of well-known isotope separation and recovery methods. Such methods were originally developed for high concentration tritium systems or to meet related hydrogen isotope separation objectives (such as purifying heavy water). Research centers around the world have contributed to this field. Commercially available processes include solution phase exchange reactions, such as Girdler-Sulfide, and various forms of liquid-solid catalytic exchange processes. These evaluations suggest that, even under favorable assumptions, costs for these separation-based systems are $10 \mathrm{x}$ to $>100 \mathrm{x}$ the costs of traditional environmental treatment systems. As a result, implementation costs are extreme with minimal risk reduction. In some cases, the systems use large amounts of energy and other undesirable collateral environmental damage. Furthermore, tritium is not destroyed in these systems, but simply separated from the "uncontaminated" water molecules, leaving a tritium rich concentrate to manage as secondary waste. As a result of these various factors, alternative methods of reducing releases and exposure have received increasing attention. Such methods include immobilization/storage to directly reduce flux and evaporation (and related methods) to reduce population exposure. Typical conclusions in these studies are:

- With additional technical development, onsite immobilization using methods other than isotope separation appear promising for appropriate sites.

- Tritium treatment based on hydrogen isotope separation may be feasible with additional technical development. Bounds on the type of progress needed to make this class of technology feasible were defined and are reflected in the vital objectives below.

Tritium is traditionally monitored by collecting a sample followed by liquid scintillation counting (or similar laboratory analysis). Sensors and detectors have been developed, but these have lacked the sensitivity to detect and quantify tritium at activities necessary to meet environmental objectives. Downscaled and field deployable lab analysis systems have been developed, but these are relatively expensive to build and deploy on a largescale basis and many do not provide detection limits that are suited to detection of plume arrival. Notable sensor and field deployable systems include those being developed for the Nevada Test Site and at SRTC. As described below, users need technical support in developing and implementing creative approaches to allow tritium monitoring under a range of environmental conditions. In particular, strategies that provide sensitive lowactivity measurements and that can be deployed in deep wells are highlighted. These 
would provide sensitive indicators of initial plume arrival and the ability to monitor tritium releases or flux when properly deployed. To be useful, the requested systems may consist primarily of a sensor or instrument, or may be based on creative sample collection and integration approaches.

\section{Vital Scientific and Technical Objectives:}

Develop technologies and strategies to reduce the impacts of tritium releases from DOE sites, specifically:

- Develop, evaluate and implement control strategies based on environmentally appropriate methods for large-scale modification of hydrologic systems (reduction of infiltration, increasing evapotranspiration, and other methods). Note that this approach does not necessarily require collection or recirculation of contaminated water - reduction of the infiltration of clean water into a system will provide a concomitant reduction in tritium release. This has a high potential for significant reductions in release for relatively low costs.

- Evaluate and implement tritium isolation or immobilization for small high-activity source areas. Note that possible techniques include, but are not limited to, physical barriers (caps, walls and floors) or immobilization of the overall plume water (freezing, and the like). When immobilizing tritium in the subsurface, displacement of water by any injected reagent/fluid will displace the tritium and spread contamination -- such methods should be carefully evaluated and justified for use on tritium plumes.

- Continue to study and implement, if justified, tritium treatment (hydrogen isotope separation) for the highest concentration environmental settings. Potential investigators should be able to document that a method is substantially different from existing methods (in terms of theoretical approach or implementation concept) and that the proposed different approach could lead to the required improvement in cost effectiveness. A theoretical cost and implementation analysis should show a reasonable probability that the approach might be usable. This analysis should consider: a) operating and maintenance costs less than about $\$ 20 / \mathrm{m}^{3}$ (including required pretreatment steps) for a typical environmental implementation, b) appropriate complexity, safety and potential capital costs for a reasonable size system, and c) minimal collateral environmental damage (e.g., reasonable energy use) that is consistent with expected risk reduction and overall national environmental policies/goals. If the analysis does not suggest a reasonable probability of significantly improving the science beyond the well-known isotope separation techniques, then tritium risk reduction investment will target alternate approaches.

Evaluate and implement methods for tritium sensors, field screening and down-well tritium measurement systems.

- Early emphasis should be on low-cost tritium screening systems that would provide a reliable early warning of plume arrival - these methods should be selected based on stakeholder acceptability, minimizing potential for frequent false positives, and reasonable cost. Deep (>1500 feet) well monitoring at the Nevada Test Site 
represents the most significant early demonstration and deployment target with - related needs at several other sites.

\section{Links to other Technical Targets:}

Acceptable progress towards this target depends on making key advances in developing and implementing improved decisionmaking and performance verification approaches as described in "Methods to Verify and Validate Performance" and "Improving the Technical Basis for Setting Remediation Goals". 


\section{Subsurface Access and Delivery}

Team: Dawn Kaback, Scott Petersen, Jack Corey, Phil Washer, Gary Brown and Glenn Bastiaans

\section{Summary of Need(s):}

Poor access, rather than poorly understood chemistry or biology, is the primary reason for poor remediation system performance at most sites. Needs for enhanced access and delivery methods potentially exist at all DOE sites and were identified by STCG organizations at seven DOE sites. The various needs are embodied in several themes:

- access under obstructions (e.g., surface or subsurface facilities),

- delivery of fluids for treatment or containment,

- improved methods for deep access,

- more cost-effective access methods in difficult geology, and

- delivery and maintenance of devices for characterization and/or monitoring.

Because of the wide range of end-user needs on this topic, the target provides an initial assessment of priorities and tabulates the relative importance of the various themes in the vital scientific and technical objectives section. The three most important themes highlighted in this cross-cutting target were access under obstructions, delivery of fluid for treatment or containment, and deep access.

\section{Relevance:}

Enhanced access and delivery technologies have significant potential to positively impact the DOE Environmental Management Program at all DOE sites by reducing costs, accelerating schedules, improving remediation performance, and filling gaps where current technologies cannot meet the needs. DOE has actively supported progress in this target area -- strong historical contributions include environmental applications of horizontal wells, cone penetromenter systems, and the like.

Several DOE sites are characterized by difficult drilling conditions controlled by the geology, such as fractured rock, cobbles, and cemented strata. Some of these sites are also impacted because accessing depths greater than 30 meters is challenging and costly. All DOE sites contain surface and subsurface obstructions and facilities below which contaminants need to be characterized, monitored, and remediated. Significant cost savings can be realized by accessing these areas through innovative technologies.

Improved access techniques may also impact the amount of drilling and characterization needed.

One of the fundamental limitations of in-situ remediation or treatment systems is access and delivery of treatment or containment agents. Enhancement of fluid delivery technologies and approaches has enormous potential to significantly improve the effectiveness of these remediation or treatment systems. Verification of the performance of these delivery tools has not been demonstrated effectively. Improved delivery and 
WSRC-RP-2002-00077

Revision 0

Page B42 of B61

maintenance of characterization and monitoring devices would also yield significant cost savings and performance enhancement opportunities, especially over the long term.

\section{Status:}

A variety of access and delivery technologies have been utilized for many years in industries other than environmental remediation. Over the last ten years, some of these technologies have been modified for environmental applications. However, more work remains to adapt these technologies to meet DOE needs, to reduce costs, and to integrate the access and delivery technologies with treatment and containment technologies.

Baseline drilling technologies include air rotary, cable tool, auger, and mud rotary. Baseline technologies utilized at a specific DOE site are dependent upon site geology, local regulations, and historical operations. The DOE sites across the nation are sited in various geologies, some of which are more difficult than others to access cost effectively (e.g., Hanford). Opportunities to utilize alternative drilling technologies exist at many DOE sites. Recent concerns related to waste minimization and the handling of investigative-derived waste have increased the opportunities for the use of alternative technologies. DOE has utilized alternative technologies such as sonic drilling and push technologies (cone penetrometer and Geoprobe) at a number of sites. Current technology development is underway to modify these technologies to expand the niche for application. Modifications to the cone penetrometer to expand its application to more difficult terrains are being pursued, including the integration of laser cutting and the dualtube percussion drilling system to the cone penetrometer. In the early 1990s, DOE supported the demonstration of cryogenic drilling. Further development, however, has not occurred, but could be useful.

Access beneath existing obstructions and facilities is a technology gap that needs to be filled and has the potential to save significant amounts of money. For example, at sites where buildings are being decontaminated and decommissioned and scheduled for removal, the ability to leave the underground foundation in place (if no significant contamination can be detected below the foundation) can result in significant cost savings. Angle drilling using technology such as sonic and auger has been utilized in an attempt to characterize beneath facilities. In the early 1990's, DOE, in partnership with private industry, invested resources in the modification of directional drilling technology for environmental applications. This technology has been utilized at more than 1000 locations across the U.S. However, significant modifications to meet DOE needs, which include sampling while drilling, emplacement of monitoring devices, and waste minimization, are needed.

Methods to enhance delivery of fluids for treatment and containment have been investigated over the last ten years. Fracturing technologies (hydraulic, pneumatic, and blast) and horizontal wells have been deployed to improve fluid delivery at numerous sites across the U.S, especially those characterized by low permeability. However, validation of the performance of these technologies remains to be done. The ability to attain cleanup goals also remains to be demonstrated. Jetting technology has been 
applied to containment situations. More technology development is needed to optimize and validate the delivery process for containment fluids. Novel delivery systems such as Prefabricated Vertical Drains [Well Injection Depth Extraction] for application at sites with low permeability has been investigated and has significant potential, if combined with appropriate fluids at these sites.

Methods to deliver characterization and monitoring devices to the subsurface have been developed and deployed over the last ten years. However, further advances, such as emplacement in horizontal wells, are needed. In addition, technologies for maintaining these devices over the long term remain to be developed.

\section{Vital Scientific and Technical Objectives:}

Target objectives map the five themes described above. Specific issues that rèquire technology development within each of the five themes are described below:

- Integration of access and delivery technologies with remediation systems and monitoring devices

- Horizontal Wells: delivery of remediation fluids, hole stability, sampling, emplacing monitoring and characterization devices, drilling deep (and accurately), reducing IDW, cost, decommissioning boreholes

- Fracturing and Jetting Delivery Technologies: validation, fundamental questions of applicability in remediation situations (continuity, validation)

- Fluid Delivery: displacement of existing fluids, nano- and microparticle delivery

- Deep access: reducing IDW, cost, hole stability

- Push Technologies: limited depth, success dependent on geologic conditions (caliche, cobbles, rock), limited application for fluid delivery

- Longevity of systems: (e.g., robust and flexible design/construction, ease of maintenance)

- Impact of access technology to the subsurface (e.g., compaction, altering chemical conditions)

\section{Sub-Target Theme Ranking:}

The above objectives were summarized as five sub-targets. These were qualitatively rated according to three criteria: DOE market size (how many sites would substantially benefit, or major impact at fewer sites); potential impact with improvements (substantial schedule/cost savings with improvement); likelihood of success (technology risk). These ratings are presented in Table 1. A brief explanation of the sub-targets follows:

- Access Under Obstructions: the ability to cost effectively access the subsurface under surface and subsurface obstructions.

- Delivery of Fluid for Treatment or Containment: the ability to effectively deliver

- remedial fluids (solutions, gases, emulsions) to the subsurface.

- Deep Access: the ability to cost-effectively access the subsurface deeper than 30 meters, especially important at Hanford, INEEL, NTS, and LANL. 
- Difficult Access: the ability to cost-effectively access the subsurface at sites with difficult terrain, such as consolidated or cemented strata, cobbles and boulders, fractures, etc.

- Delivery and Maintenance of Characterization and Monitoring Devices: the ability to emplace and maintain characterization and monitoring devices in various subsurface situations, such as vertical wells, horizontal wells, small-diameter boreholes, etc.

Table 1. Recommended Priorities for Funding of Specific Sub-Targets

\begin{tabular}{|l|l|l|l|l|}
\hline Sub-Targets & $\begin{array}{l}\text { DOE } \\
\text { Market Size }\end{array}$ & $\begin{array}{l}\text { Potential Impact } \\
\text { with } \\
\text { Improvements }\end{array}$ & $\begin{array}{l}\text { Likelihood } \\
\text { of Success }\end{array}$ & Overall Score \\
\hline $\begin{array}{l}\text { Access Under } \\
\text { Obstructions }\end{array}$ & H & M & H & H \\
\hline Delivery-Fluid & M & H & M & M \\
\hline Deep Access & H & H & L & M \\
\hline Difficult Access & H & M & L & M \\
\hline $\begin{array}{l}\text { Delivery- } \\
\text { Devices }\end{array}$ & M & L & H & L \\
\hline
\end{tabular}

L=Funding would allow some improvement

$\mathrm{M}=$ Funding would allow substantial improvement

$\mathrm{H}=$ Essential for funding

Essential Strategies to Accelerate Progress:

- Target highest priority sub-target(s) for funding.

- Utilize strategic partnerships, outside expertise, existing expertise within organization.

- Encourage DOE contractors to take advantage of lessons learned through personnel sharing at subsequent sites

\section{Links to other Technical Targets:}

Acceptable progress towards this target depends on making key advances in performance verification approaches as described in "Methods to Verify and Validate Performance". This target supports the cleanup targets, particularly those related to source zone treatment and stabilization. 


\section{Techniques and Technologies that Support Characterization}

Team: Tim O'Rourke, Caroline Purdy, Scott Petersen, Van Price, Tyler Gilmore, Phil Washer, Gary Brown and Glenn Bastiaans

\section{Summary of Need(s):}

Characterization is used in environmental remediation for initially determining contaminant distribution and concentration, guiding design of remedial actions, monitoring remediation, and verification that the remedial actions are successful. There were two major themes identified by the large number of characterization related needs statements. These were: 1) improved monitoring and sensor configurations and 2) development and implemenation of tools to meet specific needs. The themes and key issues are summarized below.

Improved Monitoring and Sensor Configurations (noninvasive, in-situ and integrating Sensors to meet a variety of objectives)

- Development and improvements and of non-invasive characterization and in-situ technologies (for cost-effectiveness, measurement of undisturbed conditions)

- Integrated, continuous measurement of large areas and volumes (addresses scaling issues)

Development and implementation of tools to meet specific needs

- Proper application of current technologies (e.g., expert systems for choosing appropriate technology, training on-site personnel)

- Development and validation sensors for key constituents

- Development and application of "just-in-time" characterization tools

- Development of field deployable technologies

- Simplification of measurement logistics (e.g., replace laboratory techniques with field technologies)

- Integration and interpretation of data (data fusion)

- Demonstration/deployment of tools developed in NABIR and EMSP projects

\section{Relevance:}

Characterization is a support activity for nearly all aspects of site cleanup, from site investigation through remediation. Higher resolution of results, additional specific contaminant information, and data that supports enhanced understanding of subsurface features (both natural and man-made) all provide opportunities to support better decisionmaking and reduce cleanup costs.

\section{Status:}

The development of characterization technologies is a mature area due to the importance that has been placed early on in understanding subsurface environments. Significant 
WSRC-RP-2002-00077

Revision 0

Page B46 of B61

characterization $R \& D$ programs have been supported by DOE and other federal agencies programs (e.g., SERDP, ESTCP, EPA's and SITE program), over the past 10-15 years. Consequently, advanced methods exist for most characterization problems, but they continue to be under used in site programs. State-of-the-art technologies that have been demonstrated and verified have not translated into the state-of-the-practice. For example, there have been field methods devised for many standard detection technologies, such as GC/MS, Raman Spectroscopy, Laser Induced Fluorescence, Laser Induced Breakdown Spectroscopy, but most of these existing field technologies are not fully mature and, thus, are rarely used in environmental projects.

There are three overarching remaining needs:

- First, in many cases the innovative technology does not provide the resolution or detection limits required to meet the project objectives or to provide adequate confidence for understanding the problem. For example, to what scale can geophysics image DNAPLs with current methods? Do the interference problems inherent in some immunoassay methods available today restrict their use? Thus, enhancing many of the current innovative technologies represents a significant opportunity.

- Second, many new innovative detection methods need to be tested and engineered for field use, operating either at the surface or in the subsurface. For example, the emerging areas of microcantilver methods or MEMS have yet to be adapted for inthe-field use. Adapting characterization technologies for field use continues to be a focus since the quick turn around in results while in the field reduces the potential of sample degradation, reduces generated laboratory waste, reduces time to make decisions, etc.

- Third, many characterization technologies are relatively new or are only used for special cases; therefore, the state of the practice in applying them lags their availability. Increased emphasis on technical assistance, development of expert systems, education and information sharing will assure that the best available technologies are considered for characterization projects.

Essential Strategy to Accelerate Progress:

- Target highest priority needs for funding

$>$ Non-invasive characterization tools for large areas and volumes

$>$ Better application of existing tools (training, data fusion, leverage science projects) 
Vital Scientific and Technical Objectives:

The vital scientific and technical objectives have been complied in the following table.

\begin{tabular}{|c|c|c|c|}
\hline Characterization Problem Area & Measurement Target & Issues & R\&D ongoing and future \\
\hline $\begin{array}{l}\text { Identification of Contaminants to bound } \\
\text { initial remediation decisions }\end{array}$ & $\begin{array}{l}\text { Metals } \\
\text { Rads } \\
\text { Organics }\end{array}$ & $\begin{array}{l}\text { Suites -Be, As } \\
\text { Sensors - Alpha, beta, Sr, H-3, Pu, U } \\
\text { Indicators for MNA }\end{array}$ & Sensors \\
\hline $\begin{array}{l}\text { Distribution of Contaminants within } \\
\text { source zones to allow selection and } \\
\text { design of remedial systems - }\end{array}$ & $\begin{array}{l}\text { Distribution in the subsurface } \\
\text { Identifying Buried Objects } \\
\text { Beneath and around structures } \\
\text { (buildings, tanks, piping, etc.) }\end{array}$ & $\begin{array}{l}\text { DNAPL distribution } \\
\text { In waste sites } \\
\text { Emerging issue with access elements for D\&D }\end{array}$ & $\begin{array}{l}\text { DNAPL toolbox - develop volumetric tools } \\
\text { and techniques (vs. point measurements) } \\
\text { Seismic - single well, cross well, surface } \\
\text { Geophysical methods for delineating buried } \\
\text { waste. } \\
\text { Remote sensing for phytotoxicity } \\
\text { Efficient under-building sampling }\end{array}$ \\
\hline Speciation data & $\begin{array}{l}\text { To support transport and toxicity } \\
\text { understanding }\end{array}$ & $\mathrm{Pu}, \mathrm{U}, \mathrm{Cr}$ & Pu environmental chemistry \\
\hline Indicators of 'natural' processes & $\begin{array}{l}\text { Pre-remediation setting important so } \\
\text { remediation can work with natural } \\
\text { processes }\end{array}$ & $\begin{array}{l}\text { Need actual ambient conditions } \\
\text { Dissolved Oxygen, } \mathrm{eH}, \mathrm{H} 2 \text { - sensitive to sampling } \\
\text { Indicators of natural attenuation }\end{array}$ & $\begin{array}{l}\text { Low-impact sampling } \\
\text { In-situ probes for redox, DO, other critical } \\
\text { parameters }\end{array}$ \\
\hline Process support measurements & $\begin{array}{l}\text { Support limits for excavation, sorting } \\
\text { or other removal activities } \\
\text { Performance or failure of } \\
\text { remediation systems }\end{array}$ & $\begin{array}{l}\alpha, \beta \text { detectors for segmented gate type technology and } \\
\text { 'near-real-time' support for excavation } \\
\text { Barrier or ISRM breakdown or breakthrough }\end{array}$ & $\begin{array}{l}\text { Opportunity for in-situ indicator parameter } \\
\text { probes }\end{array}$ \\
\hline $\begin{array}{l}\text { Physical Characterization of Sub-surface } \\
\text { - includes both the natural geologic } \\
\text { setting and man-made waste sites such as } \\
\text { trenches and burial grounds }\end{array}$ & $\begin{array}{l}\text { Chemical properties- Kd, reactivity } \\
\text { Physical properties } \\
\text { Trench boundaries } \\
\text { Buried objects } \\
\text { Fractures } \\
\text { Solution-enhanced permeability } \\
\text { Strata and sedimentary structures } \\
\text { Porosity/permeability (including } \\
\text { directionality) }\end{array}$ & $\begin{array}{l}\text { A primary objective is to understand controls on } \\
\text { migration } \\
\text { Point or grab samples - field meso-scale measurements } \\
\text { needed } \\
\text { Improved resolution of remote (geophysical) techniques } \\
\text { Knowledge support to select optimal existing techniques } \\
\text { of geophysics, aquifer testing, VZ testing... } \\
\text { Integration ("data fusion") methods for geophysics } \\
\text { toolbox }\end{array}$ & $\begin{array}{l}\text { Meso-scale test sites to validate scaling } \\
\text { methods } \\
\text { Assistance to choose and apply existing } \\
\text { technology (e.g. TA, Expert systems) } \\
\text { Improved resolution for current tools } \\
\text { New tools based on NMR, .... Other } \\
\text { electrical and mechanical properties } \\
\text { Improved resolution through data fusion or } \\
\text { co-modeling of multiple data sets (e.g. } \\
\text { gravity, magnetics, seismic, GPR)\{basic } \\
\text { science\} }\end{array}$ \\
\hline Support for post-closure stewardship & Validation and failure indicators & $\begin{array}{l}\text { Similar to some above, but emphasis on indicator } \\
\text { parameters and low-maintenance systems. We will not } \\
\text { deal with data transmission, storage, or communications } \\
\text { issues. }\end{array}$ & $\begin{array}{l}\text { Development needed to select indicators for } \\
\text { success/failure of remediation systems } \\
\text { Development needed for robust low- } \\
\text { maintenance monitoring systems }\end{array}$ \\
\hline
\end{tabular}

\section{Links to other Technical Targets:}

Acceptable progress towards this target depends on creatively exploiting observations and data from basic science studies and on making key advances in performance verification approaches as described in "Methods to Verify and Validate Performance". This target supports the cleanup targets, particularly those related to source zone treatment and stabilization. 


\section{Biogeochemical Processes that Determine Contaminant Fate}

Team: Terry Hazen, Bob Smith, Gerry Voos

\section{Summary of Needs:}

The effective implementation of remediation strategies and natural attenuation for the cleanup of DOE sites depends on understanding the basic chemical, physical and biological processes ongoing in the subsurface environment influencing the behavior of DOE-specific contaminants. These include understanding:

- Redox conditions that affect biogeochemical processes.

- The anthropogenic influences on the biogeochemistry of natural systems and extreme environments.

- Scaling issues.

- Coupled biogeochemical reactions.

\section{Relevance:}

Currently, the understanding of contaminant distribution and mobility at many sites is limited. Subsurface contaminant migration may, and in some cases, already adversely impact groundwater resources. The development of new in-situ remediation technologies such as bioremediation and monitored natural attenuation and the implementation of long-term stewardship strategies require better definition. Knowledge of the biogeochemical processes occurring in the variably saturated heterogeneous subsurface that control the fate and mobility of contaminants is critical. This knowledge will lead to a better definition of the level of remediation required at DOE sites. Specifically, research in this area will have the following impacts:

- Reduce possible negative impacts of treatment strategies.

- Provide more effective remedial strategies.

- Enable long-term stewardship and monitored natural attenuation.

- Enable in-situ treatment strategies.

- Enable the use of treatment trains and different processes in various parts of a plume.

\section{Status:}

Many agencies have been funding basic and applied research in the area of contaminant biogeochemical processes. Indeed, our understanding of the state of the science in this area is ten or more years ahead of practical field applications (Vadose Book, 2000; NRC Ward et al., 2000). Agencies currently funding research include: 1) DOE Office of Science: Basic Energy Science; Office of Biological and Environmental Research: NABIR (formerly Subsurface Science Program), EMSL; 2) DOE OST: EMSP, AS\&T program at Hanford, RTDF, ESRA at INEEL; 3) DOE Other Offices: Laboratory Directed Research and Development, Yucca Mountain; and 4) Other agencies (EPA; NSF; USDA; Sea Grant; USGS). The EMSP program alone has funded (past or present) 
78 projects in this area. Given the many agencies that are conducting research in this area, there is a great need to improve interagency coordination. Coordination of all activities in this area across DOE offices and programs, and across other agencies, would improve leveraging of SCFA efforts and avoid redundancy. SCFA should focus its efforts on practical applications that are specific to DOE EM needs. This will make remedial strategies more effective, reduce negative impacts of treatment strategies, and enable monitored natural attenuation and long-term stewardship of DOE contaminants.

\section{Vital Scientific and Technical Technology Objectives:}

Specific objectives for each of the research categories in the Summary of Needs above are presented below with DOE-important foci for each category.

1. Understanding redox Conditions that affect biogeochemical processes:

Although abundant research on biogeochemical redox processes at the laboratory scale has resulted in the delineation of mechanisms influencing contaminant behavior, the translation of these results to predict field scale processes has had limited success. A key scientific chokepoint is our ability to assess the rates of biogeochemical redox transformation occurring at contaminated and uncontaminated (e.g., down gradient) sites and the long-term persistence of redox states. Because these processes are slow relative to the time frame of monitoring, slight but significant decreases in contaminant concentrations are swamped by natural variations in monitoring records. To overcome this limitation, research leading to new strategies to define redox transformation rates is required. Such research should take advantage of the fundamental advances in areas like microbial genomics, by developing strategies to directly interrogate microbial communities regarding their physiological state and dominant electron acceptor processes. These approaches need to be highly specific, rapid, inexpensive, and in-situ or use field-stabilized samples. Development of effective approaches to determine the rate of in situ biogeochemical redox processes will enable the expanded application and inclusion of natural attenuation in the development of overall stewardship strategies:

- Determine process rates at a field scale.

- Develop direct and real-time redox measurements.

- Develop protocols for achievable redox end states.

2. Anthropogenic inputs will influence the biogeochemistry of natural systems: Emplacement of subsurface reactive barriers will influence redox conditions and groundwater flow. Injection of treatment media such as sodium dithionite may alter microbial processes and influence stability of co-contaminants. These types of injections have also proven susceptible to drilling operations in the treatment area. The extent and flux of these effects (e.g., increasing/decreasing soil water and oxygen levels) must be understood, and when possible, quantified in a variety of media for us to predict (model) remediation success. Activities such as:

a. Evaluating the effects of remediation strategies (e.g., steam injection, lixiviants, oxidizing/reducing agents) on biogeochemical processes (e.g., microbial activity, contaminant residence time and speciation); and 
WSRC-RP-2002-00077

Revision 0

Page B50 of B61

b. Evaluating the fouling of treatment systems (e.g., reactive media in permeable reactive barriers) should be conducted to help us gain this knowledge.

3. Understanding scaling issues:

The manner in which physical, chemical, and microbial processes upscale from the pore level up to soil profiles, watersheds, and groundwater basins is currently limited by lack of direct measurements of diffusion-controlled processes. While the behavior of contaminants has become better understood in batch systems, predicting their behavior in core-scale experiments and in the field remains an outstanding challenge. Subsurface heterogeneity is a main cause of this gap. Subsurface environments are strongly transport-limited, and can be categorized into advective and diffusion-limited domains. Batch studies are inherently deficient for predicting larger scale phenomena because fluids in subsurface environments are not well mixed, even at relatively small scales $\left(10^{-4}\right.$ to $\left.10^{-1} \mathrm{~m}\right)$. Core-scale investigations are equally limited since no direct information is obtained on critical intra-core structure and mechanisms controlling macroscopic observations. Direct information is specifically lacking with respect to local, diffusion-limited processes that arise from small-scale permeability variability, preferential flow paths, and soil/sediment structure. New research must be conducted at scales that allow the development of coupling between chemical, biological, and physical processes that begin to mimic the coupling that occurs in the field. Furthermore, approaches need to include experiments with controlled heterogeneity with well-defined boundary and initial conditions as well as controlled small vadose zone field trials. Research conducted at this scale will begin to bridge the gap between laboratory based mechanistic studies and field scale applications.

- Determine temporal and spatial effects on natural attenuation or treatment strategies.

- Determine longevity and behavior of natural attenuation or treatment strategies.

4. Understanding coupled biogeochemical reactions:

Studies on co-contaminant mixtures have been conducted at the laboratory scale, and for saturated advecting systems, our understanding of these processes in the heterogeneous vadose zone is another scientific choke point. New research needs to be focused on cocontaminant interactions in heterogeneous variable saturated systems subjected to both gas and fluid.

- Determine long-term stability of actinides in the environment.

- Determine co-contaminant biogeochemical interactions.

- Determine coupled reactions in heterogeneous systems.

SCFA should also 'mine' information from other DOE programs and other agency programs to maximize its investments and potentially applying major breakthroughs that may have gone unnoticed. SCFA should also more aggressively try to coordinate research activities in this area via inter-department programs and interagency programs. 


\section{Links to other Technical Targets:}

Acceptable progress towards this target will require continued basic research and approaches to link the results to the field scale through the advanced environmental modeling target. 


\section{Strongly Heterogeneous Systems}

Team: Bob Smith, Dawn Kaback, Eric Lindgren, Jay Brown

\section{Summary of Need(s):}

Strongly heterogeneous environmental systems are those that have large and sharp contrasts in hydraulic conductivity. Examples range from natural porous media such as alluvial deposits to extreme cases found in fractured rock, karst and clastic dikes. In addition to large variations in hydraulic conductivity, the scale of the heterogeneity can also vary greatly. If the scale of heterogeneity approaches the scale of a remediation, characterization, monitoring or modeling effort, deterministic understanding may be intractable. There is a need to develop and implement alternate methods to characterize and model strongly heterogeneous systems." These are the two themes highlighted below. Possible lines of inquiry include non-deterministic approaches similar to weather modeling, and integrated (rather than point) measurements designed with the geologic depositional/formational processes and post depositional changes in mind.

In strongly heterogeneous systems, interaction between advection in the highpermeability zones and contaminant diffusion into and out of adjacent low-permeability, porous zones has a strong and often dominating influence on contaminant transport rates. Unusual interactions of contaminants with coatings formed uniquely on fracture surfaces further complicate the situation. Remediation approaches based on advection (pressure gradients) often perform poorly and unpredictably under such situations and alternate remedial approaches less sensitive to strongly heterogeneous conditions (such as concentration, thermal, or electrical-gradient approaches) are needed.

\section{Relevance:}

Prediction of contaminant transport is often the primary basis for costly remedial action decisions. Poor characterization of strongly hetergeneous systems has led to ineffective remedial designs, increased or unknown risk, higher costs, and longer remediation times. In these environments, contaminant migration and/or treatment delivery are controlled by the magnitude and scale of the contrasts in hydraulic conductivity. Currently an adequate accounting of spatial variability is not attainable with respect to cost and schedule. Given that many of the active remediation approaches rely upon manipulation of in-situ advective fluxes and are especially sensitive to heterogeneity, advances in our understanding of strongly heterogeneous systems and approaches to minimize their effects are of great benefit to DOE.

\section{Status:}

Better understanding of approaches for addressing strongly heterogeneous systems are recognized as high-priority needs within DOE/EM (EMSP projects are funded), elsewhere in DOE (e.g., Yucca Mountain), other federal agencies (e.g., EPA, USGS), and industry (e.g., petroleum). Collaboration among all involved entities is required. 
Presently, dual-media deterministic modeling, which occasionally incorporates a small amount of geologic reality, represents the state-of-the-practice. However, most characterization activities at strongly heterogeneous sites are based upon approaches developed for homogeneous porous media and do not incorporate dual-media approaches.

\section{Vital Scientific and Technical Objectives:}

Develop remediation approaches that can effectively address problems related to heterogeneity of the contaminated site.

Contaminated sites that are strongly heterogeneous are a challenge to clean up because it is difficult to contact the contaminants efficiently with a flowing fluid phase. Because most current remediation methods rely on the advective transport of extrāting or reaçting liquids or gases, or on the convective transport of heat, these attempts are invariably subject to severe mass-transfer limitations, as the mobile remediation fluids bypass much of the contamination. This situation is compounded by the large degree of uncertainty associated with the characterization of heterogeneous systems, making it difficult to assess the degree of success of a remedial action. New remediation methods that depend less on advective and convective transport should be developed. As an example, diffusive transport processes, relatively insensitive to heterogeneities, are usually slower but produce more uniform flow than advective and convective transport. New remediation methods may be possible using temperature or electrical gradients. Improved understanding of biogeochemical processes may allow the widespread use of natural attenuation as an environmental management option.

Assess whether traditional deterministic approaches are valid or whether we need to develop new approaches.

Strongly hetergeneous systems are characterized by the existence and development of preferential flow paths (e.g., fractures) that dominate advective fluxes in the system. Often the effects of preferential flow paths on observed fluxes exhibit a strong dependence on the scale of observation. In such cases, traditional characterization and parameter estimation techniques that rely upon laboratory measurements and point measurements do not provide information applicable to field-scale problems. In addition, deterministic modeling approaches relying upon equivalent properties and representative elementary volumes fail because the scale of the representative elementary volume is on the order of, or larger than, the physical size of the problem of concern. Although significant efforts by a number of programs, agencies, and industry are devoted to the study of subsurface heterogeneity, significant advances in our ability to manage contaminated, strongly heterogeneous sites can be achieved by focused research on:

- Holistic characterization approaches

Such approaches would integrate a variety of characterization techniques such as tracer tests, geophysical measurements (e.g., tomography), and point measurements through the use of inverse modeling to identify subsurface features responsible for 
fluid movement. Research should be conducted at a variety of scales to capture scale-dependent behavior. Progress in this area would provide the enabling science and tools for more effective site characterization and the development of long-term monitoring strategies for strongly hetergeneous sites.

- Develop new methods for representation and prediction of strongly heterogeneous systems Experimental research is needed to address the fundamental issues of mathematical representation of fluid fluxes in strongly heterogeneous systems. This research needs to be conducted at scales that allow the development of preferential flow paths in media with complex interconnected structures and should include 1) intermediatescale experiments with well-defined heterogeneity, boundary conditions, and initial states, 2) small, highly monitored, well-controlled field trials, and 3) supporting numerical experiments. The goal of this research is to incorporate the inherent limitations resulting from imperfect and incomplete characterization and from limited understanding of the flow physics into new modeling strategies for predicting contaminant migration, implementing remediation technologies, and defining longterm monitoring strategies.

\section{Links to other Technical Targets:}

Acceptable progress towards this target will require rather specialized objectives being addressed within the advanced environmental modeling target and will support those sites where strongly heterogeneous systems are a critical barrier to progress. 


\section{Advanced Environmental Modeling}

Team: Mike Kelley, James Murphy, Doug Burns, Jim Melton, Jim Helt, Jack Parker and Terry Hazen

\section{Summary of Needs}

The primary themes associated with this target are to:

- identify and fill knowledge gaps in models of field-scale environmental processes to facilitate more accurate predictions of transport behavior at complex field sites,

- develop comprehensive protocols for optimal decisionmaking in the face of prediction uncertainty to meet remediation goals with minimum cost, and

- develop integrated modeling technologies to assess long-term site-wide transport behavior to facilitate decisionmaking for remediation and long-term stewardship.

All three of these needs support near-term remedial action decisions as well as long-term monitoring and stewardship activities.

\section{Relevance}

The majority of remedial and long-term stewardship decisions require some form of modeling to evaluate how various alternatives will perform over time. Models may range from empirical extrapolation protocols, based on bench- or pilot-scale tests, to more or less sophisticated, physically-based, numerical models. In contrast to empirical models, physically based models have the capability to predict system behavior under combinations of conditions not previously directly observed, and they provide a means to quantify prediction uncertainty due to uncertainty in model parameters for given site conditions.

Inaccurate predictions may lead decision makers to select remedial strategies and/or monitoring regimes that are ineffective, unnecessarily expensive, or both. Errors in predictions of subsurface contaminant movement generally lead to one of two situations. First, if a model over-predicts the rate of contaminant movement or under-predicts the rate of mass removal, transformation or immobilization, more aggressive and more expensive remediation may be implemented than necessary. Alternatively, if plume containment or attenuation is over-predicted, the remediation plan may fail to meet performance criteria, resulting ultimately in increased costs to rectify shortcomings and to possible undermining of public confidence.

Improved models of fundamental processes and more efficient technologies for integrated long-term site-wide modeling could reduce prediction uncertainty. However, increasing the accuracy of predictions often comes at the price of increased costs for data collection and analysis. The overall decisionmaking process must assess tradeoffs between costs and benefits of various modeling approaches as well as those of alternative remedial strategies to minimize total cost in the face of prediction uncertainty.

\section{Status}

DOE and other federal agencies have developed many computer models over the last 20 years. These models represent a significant resource with unique capabilities to evaluate 
alternative characterization, remediation and monitoring strategies, to optimize resource utilization, and to minimize long-term costs. To fully realize the potential benefits of models, certain limitations to their use must be considered:

- Intrinsic limitations - All models have limitations by virtue of the assumptions and simplifications upon which they are based. If model assumptions are inconsistent with site conditions, they may have insufficient accuracy for their intended use.

- Practical utilization issues - Models will be underutilized if they are difficult and/or costly to set up, determine/calibrate input parameters, and run or if computer resources required are prohibitive.

- Model uncertainty issues - Model uncertainty is difficult to fully assess but certain to exist. Ambiguity over the benefit of modeling in light of their uncertainty and how to factor this into decisionmaking may lead to model avoidance.

In general, the modeling process consists of the following steps: develop a conceptual model, formulate a mathematical model, implement and verify a solution approach, calibrate the model to field conditions, and assess effects of model uncertainty on decision variables. This process is, or should be, iterative in practice. The process can fall short of its potential due to failures in one or more of the foregoing steps or, due to lack of adequate iteration among the various steps.

Models of the fate and transport of certain subsurface contaminants have been employed at many DOE sites. These models often yield inaccurate and/or unreliable results due to uncertainty in model parameters or to fundamental shortcomings in our understanding and ability to quantify complex subsurface processes involving, for example, coupling of physical, chemical, and biological processes and scale-up from bench- to field-scale. It is critical to identify key processes limiting predictive ability and develop mathematical models to accurately describe the processes, while avoiding unnecessary complexity to maximize practical utility.

In typical modeling efforts, a large percentage of the total time is spent calibrating the model to site data and evaluating effects of parameter uncertainty on model predictions. Critical tradeoffs must be made between model complexity versus potential accuracy, temporal and spatial resolution versus data requirements, and complexity versus implementation cost, with careful consideration of sensitivity of decision variables to model uncertainty.

Much work has been performed in the last 20 years on the development of efficient numerical models for subsurface transport. Nevertheless, field applications of models for coupled biogeochemical processes can stress existing computational resources and consume substantial personnel time to implement, especially when uncertainty analyses are performed. Improved and portable techniques for distributed parallel computing, implementation of standardized L/O structures, and development of generic multiprogram user interfaces could assuage these limitations. 


\section{Vital Scientific and Technical Objectives}

Identify and Fill Knowledge Gaps in Models of Field-scale Environmental Processes Our current ability to predict field-scale transport behavior is limited is certain cases by incomplete understanding of processes at the bench-scale (ca. $<0.1 \mathrm{~m}$ ) scale) and in many cases by uncertainty in process quantification at the field scale (ca. $>10 \mathrm{~m})$. Some specific problems that are incompletely understood at the small scale include:

- constitutive relations for multiphase fluid retention and flow, especially for dense, high mobility, nonspreading fluids that exhibit high flow instability,

- effects of fluid saturation and saturation history, surfactants and other factors on residual NAPL interfacial area and mass transfer rates to water and gas phases, and

- modeling equilibrium and kinetically-controlled reactions involving complex biogeochemical interactions among inorganic and organic chemicals and microbes.

Scaling up these and many other processes from the bench-scale to the field-scale is even more problematic - increasingly so as the degree of complexity and heterogeneity becomes more pronounced (e.g., multiphase flow, fractured rock, rate-limited biogeochemical reactions, etc.). It is probable that in many cases, the form of the constitutive relations that governs the field-scale will be different and significantly more complex than bench-scale constitutive relations (e.g., field-scale relative permeabilities may vary not only with fluid saturation and saturation history, but with pressure gradient and rate of pressure change). Fundamental issues exist regarding the limit of the scale to which systems can be averaged without significant loss of accuracy in describing largescale behavior.

Mass transfer kinetics of organic and inorganic chemicals between immobile sorbed or residual NAPL phases and water or vapor phases is an important but poorly characterized subsurface process at the bench-scale, and to a greater degree, at the field-scale. To a great extent, these processes control the magnitude, extent and duration of dissolved and vapor phase plumes and the effectiveness of such diverse remediation technologies as groundwater extraction, forced air remediation, engineered in-situ bioremediation, and natural attenuation. While mass transfer kinetics is governed at the bench-scale by pore geometry and interfacial surface area, at the field-scale, heterogeneities in the distributions of contamination and fluid velocities become increasingly important. Mass transfer rates may be expected to change with mean fluid velocity and with changes in contaminant mass remaining. Reactions of surfactants, co-solvents and other chemicals with contaminants may also markedly affect mass transfer rates.

Most computer models simulate source zones by specifying the fluid phase contaminant concentration or mass transfer rate as a specified value over time. More sophisticated models consider first-order mass transfer with constant mass transfer coefficients. Dependence of mass transfer coefficients on temporally varying factors is rarely considered due to a fundamental lack of understanding on the functional form of fieldscale mass transfer relations. Without such knowledge, it is difficult if not impossible to optimize remediation system operation to maximize efficiency or to predict long-term 
behavior of source zones. The development of models to simulate the functional dependence of mass transfer rates on key variables is a critical need.

Users of subsurface modeling results are interested in how physical, chemical, and biological processes interact to produce given end states. For example, a given contaminant in the subsurface may be affected by advection and chemical transformation, but it is the combination of these processes and their spatial and temporal variabilities that produce the contaminant concentration that could impact a receptor. Thus, research into the coupling among processes is often as important or more so than research into the individual processes themselves.

Laboratory-scale research results must be scaled up to be useful in field-scale models. For example, information about how mineral coatings on individual fractures affect the movement of contaminants through the fractures may be of limited use to models that evaluate the movement of contaminants through entire fracture networks. Further work is needed to identify the processes that affect contaminant movement at a scale consistent with practically usable models.

\section{Develop Comprehensive Protocols for Decisionmaking subject to Prediction Uncertainty}

All subsurface fate and transport models have limitations. The reasons these limitations exist arise from the fact that there are myriad physical, chemical, and biological processes that operate in the subsurface environment, and these processes operate at varying spatial and temporal scales. Developing codes that capture all processes at all scales with zero uncertainty is impossible. By definition, models involve approximations and these approximations produce uncertainties. Understanding the impacts of a model's limitations is often the key to developing a successful modeling analysis.

Several sources of uncertainty can impact modeling results, including:

- intrinsic model uncertainty (i.e., is the mathematical model capable of accurately describing dominant processes at the site?),

- parameter uncertainty (i.e., what is the effect of uncertainty in estimated model parameters on model predictions?), and

- numerical error (i.e., what are the effects of deviations from the exact solution of the mathematical problem due to numerical approximations?).

Techniques have been developed to quantify uncertainties in model results. These methods generally focus on uncertainty in primary model results, such as concentration at a given location, maximum plume length, time to reach a specified cleanup goal, etc. In some cases, a further step is taken to compute the uncertainty in costs associated with various alternatives. While such results are useful, they do not squarely address the ultimate problem of model uncertainty - namely, that decisions must be made despite the fact that uncertainty exists and cannot be eliminated. Methods must be developed to optimize the decisionmaking process in its full spectrum in the face of uncertainty. Such a methodology should address a variety of issues, including

- quantification of expected costs associated with non-optimal remedial strategy selection and design given uncertainty in system performance, 
- determination of expected monetary benefits of various characterization, pilot testing and research initiatives in terms of reducing non-optimal design costs,

- determination of optimal model complexity that yields a marginal cost-benefit considering tradeoffs of modeling cost versus accuracy, and

- evaluation of monetary benefits of phased approaches to characterization, modeling, design and operational optimization in which operational data from interim systems are utilized to refine later decisions.

Development of an integrated and holistic approach to remediation decisionmaking that explicitly considers uncertainty in predictions of system behavior would likely result in large cost savings across the entire DOE complex.

\section{Develop Integrated Modeling Technologies to Assess Long-Term Site-wide Transport} Most of the present generation of computer models were written to be executed on conventional single-processor computers with relatively low memory and processor speeds, which limit the ability to address long-term, large-scale problems with high resolution, to consider effects of highly coupled biogeochemical and physical processes, or to fully evaluate effects of model uncertainty on model results and the decisionmaking process. The availability of supercomputing resources within DOE and the potential for dramatic increases in computational capability using distributed and parallel computing platforms offer the means to employ models to much greater advantage than has been possible in the past, with modest investments in code modifications to optimize parallel processing performance.

Due to the large size and complex types and distributions of contaminants at many DOE sites, a number of remedial technologies and strategies will be used on various portions of sites and at different times. It is likely that a variety of different models applied at a range of time and space scales will be needed to address all issues at large, complex sites. To perform such analyses in an integrated manner, such that interactions among various technologies and decisions are captured, will require communication among different models. Such communication would be facilitated by the development and implementation of standardized model input/output $(\mathrm{V} / \mathrm{O})$ structures and by generic multiprogram user interfaces. These could enable users to implement sub-models on limited time and/or space scales to address specific remediation issues, while maintaining consistency with site-wide behavior affected (purposely or inadvertently) by other actions.

Improvements in existing models and possible development of new more sophisticated models is likely to be a gradual process over time. However, the benefits of these advances would be substantially leveraged by the implementation of standardized (if evolving) I/O structures and user interfaces in the short term with implementation on a test site to evaluate and demonstrate the approach. 


\section{Links to other Technical Targets:}

Acceptable progress towards this target is the most commonly cited link to meet almost all of the other technical targets. This target requires information from the biogeochemistry, heterogeneous system, and other science providing targets. 
WSRC-RP-2002-00077

Revision 0

Page B61 of B61 
WSRC-RP-2002-00077

Revision 0

Page $\mathrm{C} 0$ of $\mathrm{C} 5$

\section{Appendix C}

Information on the First Meeting in Golden CO 


\section{SCFA Strategic Target Development}

\section{Colorado Pilot Meeting:}

The SCFA has initiated a strategic planning effort, a "Strategic Target" Team, to support efficient and effective program management. The primary goal of this effort is to document a technical foundation and vision for the SCFA. We view this as an important activity to expand the many objective successes of the focus area's programs and organizations. For this process to be successful, we have assembled a small, but diverse, group of scientists each with a demonstrated ability to work cooperatively and

strategically. The group of about 21 people will be comprised of broad based technical experts from across the country. These experts will represent DOE labs, other agencies, scientific academies, universities, industry and others.

We intend to generate a few short descriptions to assist SCFA - the "Strategic Targets". These descriptions will capture and distill the voluminous detailed input data. The inputs include STCG needs statements, on-going and past work, work by other

agencies/organizations, and the like. The team will then distill the data into a format that is useful as a tool for decisionmaking. We anticipate that each target will be 2-3 pages long. The structure will be developed by the team and will include sections such as summary statement, status/issues, and vital technology objectives and bounds (see sample above).

At the opening meeting, the Team will define the nature and content of a product called a Strategic Target. The team will then define the titles/topics for these targets. Drafts of the targets will be written at the meeting by small teams with input from the entire group. In this way, the process is compact and rapid, but should provide critical and responsive technical input to SCFA. 


\section{SCFA Strategic Target Development}

\section{Charter:}

The Subsurface Contaminants Focus Area (SCFA), as part of its strategic planning process, proposed implementation of a Strategic Targets Team to provide efficient and effective technical input to support future program development and management. The team will consist of experienced scientists and other technical experts who have demonstrated the ability to work cooperatively and strategically. The team is comprised of representatives from DOE national laboratories and major DOE sites, as well as other complementary experts.

The goal of this initiative is to assess soil and water remediation issues and identify and describe a set of technical targets within the SCFA work scope that can be used as the basis for future SCFA technical program development and management.

\section{Technical Targets}

The Team will define the structure and content of Technical Targets within broad guidelines. For planning purposes, Targets should embody the following characteristics:

- Complex-wide perspective

- Clearly identify the underlying technical challenge that needs to be addressed to make progress

- Meeting the Target should significantly improve some combination of cost, risk, and schedule

- Concentrate on technical areas where enough progress can be anticipated to "make a significant difference"

- Descriptions should be limited to 2-3 pages and should be:

- Understandable

- Concise

- $\quad$ Strong technical focus

- Practical

- $\quad$ Can be related to manageable SCFA projects that, when accomplished, will satisfy the technical target 


\author{
SCFA LEAD LAB \\ STRATEGIC TARGETS MEETING \\ Holiday Inn - West \\ 14707 W. Colfax Av \\ Golden, $\mathrm{CO}$ \\ July 23-27, 2001
}

\begin{tabular}{|c|c|c|}
\hline \multicolumn{3}{|c|}{ MONDAY, JULY 23} \\
\hline \multicolumn{3}{|c|}{$\begin{array}{l}\text { Objectives: } \\
\text { - Describe meeting objectives } \\
\text { - Provide background information } \\
\text { - } \text { Allow participants to highlight issues that are important to them } \\
\text { - Define strategic targets and discuss their general principles, alternative formulations and character } \\
\text { - Work through an example strategic target; make assignments for remaining strategic targets }\end{array}$} \\
\hline TIME & TOPICS & LEAD \\
\hline $8: 30 \mathrm{am}$ & Welcome and Introductions & Jack Corey \\
\hline $8: 45 \mathrm{am}$ & $\begin{array}{l}\text { "This is the Ball" } \\
\text { - Why are we here? } \\
\text { - What's the challenge to us? } \\
\text { - How will the results be used? }\end{array}$ & Brian Looney \\
\hline $9: \overline{a m}$ & $\begin{array}{l}\text { Setting the Stage -- Inputs to Potential Strategic Targets } \\
\text { - Overview of SCFA Final Draft Strategic Plan (10 min.) } \\
\text { - Product Line strategic planning (30 min) } \\
\text { - Roadmapping efforts - progress and status (20 min.) }\end{array}$ & $\begin{array}{l}\text { Emily Charoglu } \\
\text { Tom Early, Jay } \\
\text { Brown, Jody Waugh, } \\
\text { INEEL }\end{array}$ \\
\hline $10: 05 \mathrm{am}$ & Break & \\
\hline $10: 20$ am & $\begin{array}{l}\text { Setting the Stage -- Inputs to Potential Strategic Targets (cont.) } \\
\text { - Analysis of site science and technology needs ( } 20 \mathrm{~min} .) \\
\text { - Summary of heavy hitters from site needs assessment }(10 \mathrm{~min} .) \\
\text { - } \quad \text { Bechtel's analysis of high priority needs at its sites ( } 20 \mathrm{~min} .)\end{array}$ & $\begin{array}{l}\text { Van Price } \\
\text { Janice Imrich } \\
\text { Scott Petersen }\end{array}$ \\
\hline $11: 10 \mathrm{am}$ & $\begin{array}{l}\text { Group Discussion of Other Needs and Drivers for Strategic Targets } \\
\text { - Emerging issues for the vadose zone } \\
\text { - Emerging issues for groundwater } \\
\text { - Emerging issues for surface water/long-term stewardship } \\
\text { - Other emerging issues }\end{array}$ & \\
\hline $11: 55 \mathrm{am}$ & Summary of consensus from morning discussions and goals for the week & \\
\hline $12: 00 n$ & Lunch & \\
\hline $1: 00 \mathrm{pm}$ & $\begin{array}{l}\text { What's a "Strategic Target"? } \\
\text { - Proposed definition of "strategic target" } \\
\text { - Suggested principles \& criteria for defining potential strategic targets } \\
\text { - Group discussion of and consensus on principles and criteria }\end{array}$ & $\begin{array}{l}\text { Brian Looney } \\
\text { Brian Looney }\end{array}$ \\
\hline $1: 45 \mathrm{pm}$ & $\begin{array}{l}\text { Potential Strategic Targets } \\
\text { - } \quad \text { Priming the pump - example potential strategic targets } \\
\text { - } \quad \text { Round-robin brainstorming of additional potential strategic targets }\end{array}$ & Brian Looney \\
\hline $3: 00 \mathrm{pm}$ & Break & \\
\hline $3: 45 \mathrm{pm}$ & $\begin{array}{l}\text { Potential Strategic targets (cont.) } \\
\text { - } \quad \text { Continue round-robin brainstorming } \\
\text { - } \quad \text { Summarize consensus of strategic targets to write }\end{array}$ & \\
\hline $4: 30 \mathrm{pm}$ & Example Strategic Target - Work through the format and content & Brian Looney \\
\hline $5: 15 \mathrm{pm}$ & Work Assignments for Strategic Targets & \\
\hline $5: 30 \mathrm{pm}$ & Recess for Day & \\
\hline
\end{tabular}




\section{TUESDAY, JULY 24}

\section{Objectives:}

- Develop summary, main points, and section outlines for first batch of targets

- Group discussion and suggestions for outlines

- Begin preparation of draft write-ups for first batch of targets

\begin{tabular}{|l|l|c|}
\hline \multicolumn{1}{|c|}{ TIME } & \multicolumn{1}{|c|}{ TOPICS } & LEAD \\
\hline $8: 00 \mathrm{am}$ & Review first day's activities & Brian Looney \\
\hline $8: 15 \mathrm{am}$ & $\begin{array}{l}\text { Working session for each small group to develop summary, main points, } \\
\text { and section outlines for a strategic target from first batch }\end{array}$ & \\
\hline $11: 15 \mathrm{am}$ & $\begin{array}{l}\text { Reconvene } \\
\text { Summarize status and/or problems encountered by small groups } \\
\text { Discuss any process improvements }\end{array}$ & \\
\hline $11: 30$ & Lunch & \\
\hline $12: 30 \mathrm{pm}$ & Working session for small groups to finish outlines & \\
\hline $1: 30 \mathrm{pm}$ & $\begin{array}{l}\text { Reconvene - each group presents summary, main points, and outline; all } \\
\text { discuss issues and suggestions (15 min. per target) }\end{array}$ & \\
\hline $3: 30 \mathrm{pm}$ & $\begin{array}{l}\text { Working session for small groups to prepare full draft write-ups for first } \\
\text { batch of targets }\end{array}$ & \\
\hline $5: 00 \mathrm{pm}$ & Recess for Day & \\
\hline
\end{tabular}

\section{WEDNESDAY, JULY 25}

\section{Objectives:}

- Finish draft write-ups for first batch of targets

- Group review and discussion of first batch of write-ups

- Begin development of summary, main points, and outlines for second batch of targets

\begin{tabular}{|l|l|l|}
\hline \multicolumn{1}{|c|}{ TIME } & \multicolumn{1}{|c|}{ TOPICS } & LEAD \\
\hline $8: 00 \mathrm{am}$ & Review second day's activities and plan for the day & \\
\hline $8: 15 \mathrm{am}$ & $\begin{array}{l}\text { Working session for small groups to prepare full draft write-ups for first } \\
\text { batch of targets }\end{array}$ & \\
\hline $11: 15 \mathrm{am}$ & $\begin{array}{l}\text { Reconvene } \\
\text { Summarize status and/or problems encountered by small groups } \\
\text { Discuss any process improvements }\end{array}$ & \\
\hline $11: 30 \mathrm{n}$ & Lunch & $\begin{array}{l}\text { Reconvene - each group presents full write-up for target; all discuss issues } \\
\text { and suggestions (30 min. per target) }\end{array}$ \\
\hline $12: 30 \mathrm{pm}$ & Review assignments for remaining strategic targets & \\
\hline $4: 00 \mathrm{pm}$ & $\begin{array}{l}\text { Working session for each small group to develop summary, main points, } \\
\text { and section outlines for a strategic target from second batch }\end{array}$ & \\
\hline $4: 15 \mathrm{pm}$ & & \\
\hline $5: 30 \mathrm{pm}$ & Recess for Day & \\
\hline
\end{tabular}


THURSDAY, JULY 26

\section{Objectives:}

- Finish summary, main points, and section outlines for second batch of targets

- Group discussion and suggestions for outlines

- Prepare full draft write-ups for second batch of targets

\begin{tabular}{|l|l|l|}
\hline \multicolumn{1}{|c|}{ TIME } & \multicolumn{1}{|c|}{ TOPICS } & LEAD \\
\hline $8: 00 \mathrm{am}$ & Review third day's activities and plan for the day & \\
\hline $8: 15 \mathrm{am}$ & $\begin{array}{l}\text { Working session for each small group to finish outlines for a strategic target } \\
\text { from second batch }\end{array}$ & \\
\hline $10: 30 \mathrm{pm}$ & $\begin{array}{l}\text { Reconvene - each group presents summary, main points, and outline; all } \\
\text { discuss issues and suggestions (15 min. per target) }\end{array}$ & \\
\hline $12: 30$ & Lunch & \\
\hline $1: 30 \mathrm{pm}$ & $\begin{array}{l}\text { Working session for each small groups to prepare a full draft write-up for its } \\
\text { target from second batch }\end{array}$ & \\
\hline $5: 00 \mathrm{pm}$ & Recess for Day & \\
\hline
\end{tabular}

\section{FRIDAY, JULY 27}

\section{Objectives:}

- Discuss draft write-ups for second batch of targets

- Discuss recommendations

- Define homework assignments

\begin{tabular}{|l|l|c|}
\hline \multicolumn{1}{|c|}{ TIME } & \multicolumn{1}{c|}{ TOPICS } & LEAD \\
\hline 8:00 am & $\begin{array}{l}\text { Reconvene - each group presents full write-up for second batch target; all } \\
\text { discuss issues and suggestions (30 min. per target) }\end{array}$ & \\
\hline 11:00 am & $\begin{array}{l}\text { Wrap-up } \\
\text { Discussion of recommended strategic targets, priorities, and how these } \\
\text { should be used by SCFA }\end{array}$ & \\
& $\begin{array}{l}\text { Definition of homework assignments and deadlines } \\
\text { Discussion of usefulness of this process for strategic planning }\end{array}$ & \\
\hline $12: 00 \mathrm{n}$ & Adjourn & \\
\hline
\end{tabular}

